\title{
A Teia Browniana radial
}

\author{
León Alexander Valencia Henao
}

\section{TESE APRESENTADA $\mathrm{AO}$ Instituto de MATEMÁticA E EstatísticA DA Universidade De SÃo Paulo PARA OBTENÇÃO DO TÍTULO $\mathrm{DE}$ Doutor EM CIÊNCIAS}

\author{
Programa: Estatística \\ Orientador: Prof. Dr. Luiz Renato Gonçalves Fontes \\ Co-Orientador: Prof. Dr. Cristian Favio Coletti
}

Durante o desenvolvimento deste trabalho o autor recebeu auxílio financeiro do CNPq

São Paulo, Abril de 2012. 


\section{A Teia Browniana radial}

Este exemplar corresponde à redação

final tese devidamente corrigida

e defendida por León Alexander Valencia Henao

e aprovada pela Comissão Julgadora.

Banca Examinadora:

- Prof. Dr. Luiz Renato Gonçalves Fontes - IME-USP.

- Prof. Dr. Cristian Favio Coletti - UFABC.

- Prof. Dr. Glauco Valle da Silva Coelho - UFRJ.

- Prof. Dr. Sebastian Pablo Grynberg - UBA.

- Prof. Dr. Christophe Frederic Gallesco - IMECC. 


\section{Agradecimentos}

Ao professor meu orientador Prof. Dr. Luiz Renato Gonçalves Fontes, pelo apoio, por sua paciência, por sua confiança e especialmente pelo gran exemplo de profissionalismo e integridade.

Ao professor meu co-orientador Prof. Dr. Cristian Coletti, pelo apoio, por sua paciencia e especialmente por seus valiosos conselhos.

A todos os profesores de IME, com os quais tive o prazer de ter assistido aulas, especialmente ao Prof. Dr. Fabio Machado pela ajuda durante nesses anos todos.

A minha esposa Paola, pelo amor e enorme compreensão.

A minha familia pelo grande amor e carinho.

Aos meus amigos do IME pela companhia, por sua paciência e pelos conselhos.

Ao grande povo Brasileiro, sempre feliz, sempre sorrindo.

A minha filha Mariana e a minha esposa Paola. 


\section{Resumo}

\section{A Teia Browniana radial}

Introduzimos uma familia de trajetórias aleatórias coalescentes com certo tipo de comportamento radial a qual chamaremos de Teia Poissoniana radial discreta. Mostramos que o limite fraco na escala difusiva desta familia é uma familia de trajetórias aleatórias coalescentes que chamaremos de Teia Browniana radial. Por fim, caraterizamos o objeto limite como um mapeamento continuo da Teia Browniana restrita num subconjunto de $\mathbb{R}^{2}$.

Palavras-chave: Teia Browniana, limite de escala, convergência fraca. 


\section{Abstract}

\section{The Radial Brownian Web}

We introduce a family of coalescing random paths with certain kind of radial behavior. We call them the discrete radial Poisson Web. We show that under diffusive scaling this family converges in distribution to a family of coalescing random paths which we call radial Brownian Web. Finally, we characterize the limiting object as a continuous mapping of the Brownian Web restricted to a subset of $\mathbb{R}^{2}$.

Keywords: Brownian Web, scaling limit, weak convergence. 


\section{Sumário}

$\begin{array}{lc}\text { Lista de Figuras } & \text { ix }\end{array}$

1 Introdução 1

2 A Teia Browniana restrita $\quad 3$

2.1 Caracterização e convergência à Teia Browniana . . . . . . . . . . . . . . 3

2.2 Teia Browniana restrita . . . . . . . . . . . . . . . . . . 6

3 Teia Poissoniana radial discreta $r$

3.1 Descrição detalhada do modelo . . . . . . . . . . . . . . . . . . . . . . . 9

3.2 Propriedades da Teia Poissoniana Radial Discreta. . . . . . . . . . . . . . . . 13

3.3 Enunciado do Teorema principal . . . . . . . . . . . . . . . . . . 21

4 Aproximações do modelo $\quad 23$

4.1 Primeira aproximação. . . . . . . . . . . . . . . . . . . . . . . . . . . . . . . . . . .

4.2 Segunda aproximação . . . . . . . . . . . . . . . . . . 26

4.3 Terceira aproximação . . . . . . . . . . . . . . . . . . . . 27

4.4 Convergência de uma única trajetória . . . . . . . . . . . . . . 31

4.5 Transformação adequada. . . . . . . . . . . . . . . . . . . . 33

5 Prova do teorema principal $\quad 37$

5.1 Tempo de Coalescência . . . . . . . . . . . . . . . . . . . . 37

5.2 Condição $(\mathrm{I}) \ldots \ldots \ldots \ldots \ldots \ldots \ldots \ldots \ldots \ldots$

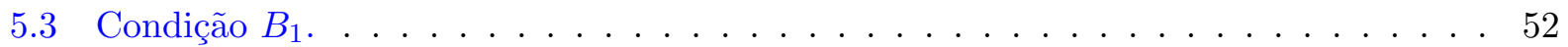

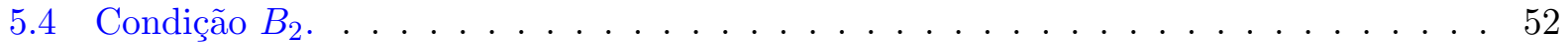

5.5 Prova do Teorema principal . . . . . . . . . . . . . . 56

$\begin{array}{ll}\text { A Convergência em distribuição } & 57\end{array}$

$\begin{array}{lr}\text { B Alguns resultados } & 67\end{array}$

$\begin{array}{ll}\text { Referências Bibliográficas } & 71\end{array}$ 


\section{Lista de Figuras}

1.1 Descrição . . . . . . . . . . . . . . . . . . . . . . . 2

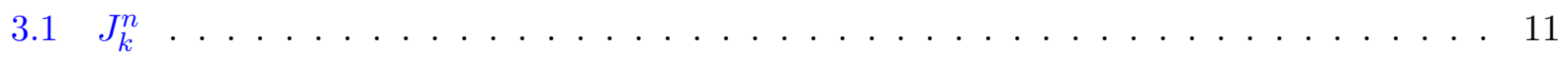

3.2 Pontos especiais . . . . . . . . . . . . . . . . . . . . . . 12

3.3 Teia Poissoniana radial discreta . . . . . . . . . . . . . . . . 13 


\section{Capítulo 1}

\section{Introdução}

Inicialmente vamos fazer uma descrição informal e simplificada do modelo e dos resultados. No capitulo 2 fazemos o detalhamento necessário e enunciamos nosso resultado de forma precisa. Considere $n$ circuferências em $\mathbb{R}^{2}$ com centro na origem e raios $n, n-1, n-2, \ldots, 1$ respectivamente. Sobre cada circunferência consideremos um processo de Poisson de taxa 1 independente dos demais. Cada ponto de Poisson de cada circunferência se liga linearmente ao ponto mais próximo da circunferência interior seguinte -quando não houver tal ponto, então procuramos um ponto na circunferência interior seguinte- e assim sucessivamente até a origem. Nesta forma, obtemos uma familia de curvas poligonais -que chamaremos momentaneamente de "caminhos"- ligando os pontos de Poisson à origem. Veja a Figura 1.1 para uma representação esquemática de uma realização do modelo.

O objetivo deste trabalho a grosso modo é obter o limite de escala para uma subfamilia desta familia de curvas numa escala difusiva onde a direção radial cumpre o papel do tempo, e a direção angular cumpre o papel do espaço.

A familia de curvas limite está relacionada com a Teia Browniana por meio de uma transformação.

A motivação deste trabalho é a Árvore geradora radial de Bacelli e Bordenave [8]. Aquele modelo é também uma familia de curvas poligonais conectando pontos de um processo de Poisson no disco de raio $n$ (mais um ponto na origem), de tal forma que cada ponto $P$ se conecta ao ponto de Poisson mais próximo no disco aberto com centro na origem e cuja fronteira contém $P$, sucessivamente até a origem. Este modelo apresenta dependência, em particular falta de Markovianidade das trajetórias, que dificulta sua análise.

O ponte em comum com nosso modelo é a componente radial. Um segundo objetivo, é entender o efeito de essa componente em nosso modelo.

A tese esta dividida da seguinte forma. No capitulo 2 fazemos uma breve descrição da Teia Brow- 
niana [1] para introduzir um objeto que chamaremos a Teia Browniana restrita (TBR) de uma forma natural. A TBR resulta sendo uma restrição da Teia Browniana sobre conjuntos da forma $\mathbb{R} \times[r, s]$, com $-\infty<r<s<\infty$. No capítulo 3 fazemos uma descrição formal de nosso modelo, que chamaremos Teia Poissoniana radial discreta (TPRD), mostramos algumas propriedades importantes e enunciamos de forma sucinta o teorema principal deste trabalho. No capítulo 4 estudamos familias de trajetórias, que chamaremos de "aproximações" a nosso modelo, que ajudam a entender o efeito da componente radial assim como também a encontrar o limite fraco na escala difusiva da TPRD. Finalmente, no capítulo 4 desenvolvemos todas as ferramentas para provar o teorema principal assim como a prova mesma do teorema, isto é, a convergência fraca na escala difusiva da TPRD a um mapeamento continuo da TBR.

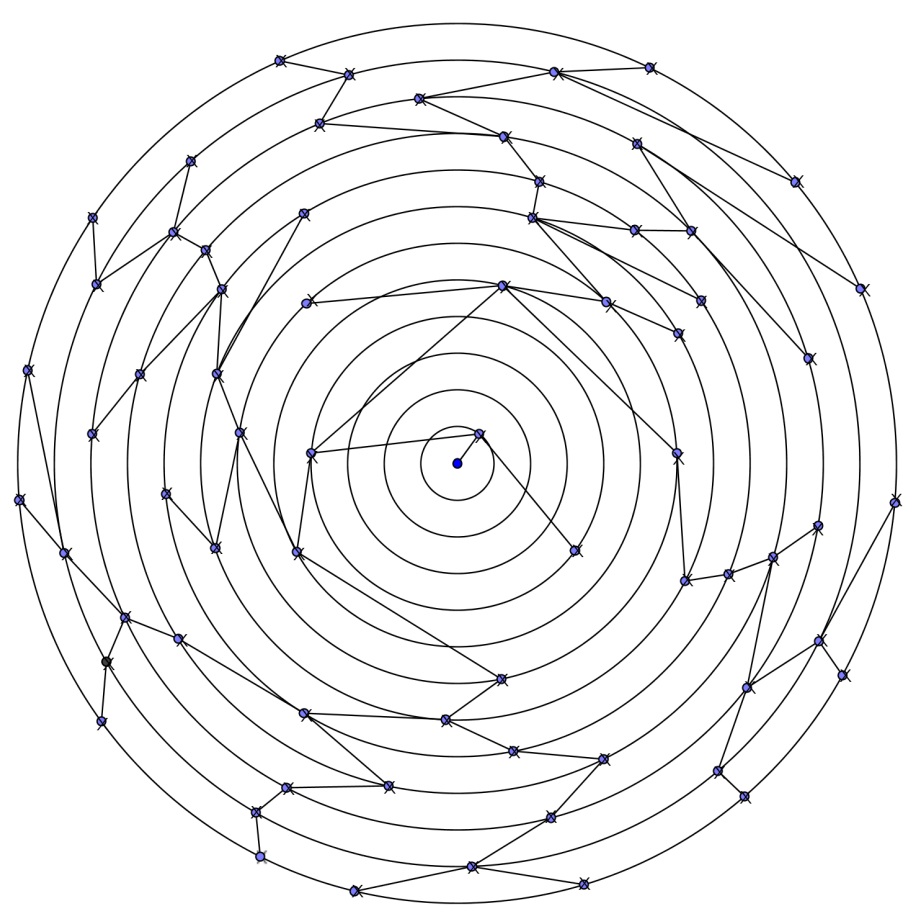

Figura 1.1: Descrição 


\section{Capítulo 2}

\section{A Teia Browniana restrita}

Como anticipamos na introdução, a Teia Browniana tem um papel muito importante neste trabalho. Definiremos um objeto chamado Teia Browniana restrita, que pode se pensar como uma restrição da Teia Browniana em conjuntos da forma $\mathbb{R} \times[r, s]$ com $-\infty<r<s<\infty$.

A continuação faremos uma breve descrição da Teia Browniana, para logo introducir a Teia Browniana restrita de forma mais natural.

\subsection{Caracterização e convergência à Teia Browniana}

Seja $\left(\overline{\mathbb{R}}^{2}, \rho\right)$ a compactificação de $\mathbb{R}^{2}$ pela métrica $\rho$ dada por

$$
\rho\left(\left(x_{1}, t_{1}\right),\left(x_{2}, t_{2}\right)\right)=\left|\frac{\tanh \left(x_{1}\right)}{1+\left|t_{1}\right|}-\frac{\tanh \left(x_{2}\right)}{1+\left|t_{2}\right|}\right| \vee\left|\tanh \left(t_{1}\right)-\tanh \left(t_{2}\right)\right| .
$$

$\overline{\mathbb{R}}^{2}$ pode ser pensado como a imagem de $[-\infty, \infty] \times[-\infty, \infty]$ pela função

$$
(x, t) \rightsquigarrow(\Phi(x, t), \Psi(t)) \equiv\left(\frac{\tanh (x)}{1+|t|}, \tanh (t)\right) .
$$

Para $t_{0} \in[-\infty, \infty]$ seja $C\left[t_{0}\right]$ o conjunto das funções $f$ de $\left[t_{0}, \infty\right]$ a $[-\infty, \infty]$ tais que $\Phi(f(t), t)$ é continua. Com isso, definimos

$$
\Pi=\cup_{t_{0} \in[-\infty, \infty]} C\left[t_{0}\right] \times\left\{t_{0}\right\},
$$

sendo que $\left(f, t_{0}\right) \in \Pi$ representa um caminho em $\overline{\mathbb{R}}^{2}$ começando em $\left(f\left(t_{0}\right), t_{0}\right)$. Para $\left(f, t_{0}\right) \in \Pi$, denotamos por $\hat{f}$ a função que estende $f$ a todo $[-\infty, \infty]$ definindo $f(t)=f\left(t_{0}\right)$ para $t<t_{0}$. Assim, definimos

$$
d\left(\left(f_{1}, t_{1}\right),\left(f_{2}, t_{2}\right)\right)=\sup _{t}\left|\Phi\left(\hat{f}_{1}(t), t\right)-\Phi\left(\hat{f}_{2}(t), t\right)\right| \vee\left|\Psi\left(t_{1}\right)-\Psi\left(t_{2}\right)\right| .
$$

Nestas condições, $(\Pi, d)$ é um espaço métrico completo separável. 
Seja agora $\mathcal{H}$ o conjunto dos subconjuntos compactos de $(\Pi, d)$, com $d_{\mathcal{H}}$ a métrica de Hausdorff induzida, i.e.,

$$
d_{\mathcal{H}}\left(K_{1}, K_{2}\right)=\sup _{g_{1} \in K_{1}} \inf _{g_{2} \in K_{2}} d\left(g_{1}, g_{2}\right) \vee \sup _{g_{\in} K_{2}} \inf _{g_{1} \in K_{1}} d\left(g_{1}, g_{2}\right) .
$$

Temos então que $\left(\mathcal{H}, d_{\mathcal{H}}\right)$ é também um espaço métrico completo separável.

Seja $\mathcal{F}_{\mathcal{H}}$ a $\sigma$-álgebra associada à métrica $d_{\mathcal{H}}$.

Teorema 2.1.1 Existe uma variável aleatória $\overline{\mathcal{W}}$ com valores em $\left(\mathcal{H}, \mathcal{F}_{\mathcal{H}}\right)$ cuja distribuição está univocamente determinada pelas três propriedades a seguir:

1. Para qualquer ponto determinístico $(x, t) \in \mathbb{R}^{2}$ existe, quase certamente, um único caminho $W_{x, t}$ começando em $(x, t)$.

2. Para qualquer número determinístico $n$ e $\left(x_{1}, t_{1}\right), \ldots,\left(x_{n}, t_{n}\right) \in \mathbb{R}^{2}$ a lei conjunta de $W_{x_{1}, t_{1} \ldots \text {, }}$ $W_{x_{n}, t_{n}}$ é a lei de movimentos Brownianos coalescentes (com constante de difusão unitária).

3. Para qualquer subconjunto denso enumerável $\mathcal{D}$ de $\mathbb{R}^{2}$, quase certamente, $\overline{\mathcal{W}}$ é o fecho em $\left(\mathcal{H}, \mathcal{F}_{\mathcal{H}}\right)$ de $\left\{W_{x, t}:(x, t) \in \mathcal{D}\right\}$.

Dando continuidade, mostraremos uma forma de construir a teia Browniana. Seja $(\Omega, \mathcal{F}, \mathbb{P})$ o espaço de probabilidade onde está definida uma família independente e identicamente distribuída (i.i.d.) de Movimentos Brownianos. Seja $\mathcal{D}=\left\{\left(x_{j}, t_{j}\right), j \geq 1\right\}$ um subconjunto denso de $\mathbb{R}^{2}$ e

$$
W_{j}(t)=x_{j}+B_{j}\left(t-t_{j}\right), t \geq t_{j}
$$

um caminho Browniano começando em $x_{j}$ no instante $t_{j}$.

Agora, especificando regras de coalescência, construímos caminhos Brownianos coalescentes a partir da família de caminhos $\left\{W_{j}\right\}_{j \geq 1}$. Quando dois caminhos se encontram pela primeira vez, coalescem em um caminho, que é aquele do movimento Browniano com o menor índice. Os caminhos coalescentes serão denotados por $\tilde{W}_{j}, j \geq 1$. Formalmente, definimos

$$
\tilde{W}_{1}=W_{1}
$$

e para $j \geq 2$, tomamos $\tilde{W}_{j}$ a aplicação de $\left[t_{j}, \infty\right)$ em $\mathbb{R}$ definida da seguinte forma. Seja

$$
\begin{aligned}
\tau_{j} & =\inf \left\{t \geq t_{j}: W_{j}(t)=\tilde{W}_{i}(t) \text { para algum } 1 \leq i<j\right\} \\
I_{j} & =\min \left\{1 \leq i<j: W_{j}\left(\tau_{j}\right)=\tilde{W}_{i}\left(\tau_{j}\right)\right\}
\end{aligned}
$$

o primeiro instante quando $W_{j}$ encontra algum caminho $\tilde{W}_{i}$ com $i<j$, e a menor etiqueta entre tais caminhos. Definimos agora 


$$
\begin{aligned}
\tilde{W}_{j}(t) & =W_{j}(t), \text { se } t_{j} \leq t \leq \tau_{j} \\
& =\tilde{W}_{I_{j}}(t), \text { se } t>\tau_{j}
\end{aligned}
$$

A seguir definimos $\mathcal{W}(\mathcal{D})$, o esqueleto da teia Browniana, começando do conjunto $\mathcal{D}$ da seguinte forma:

$$
\begin{aligned}
\tilde{\mathcal{W}}_{k} & =\tilde{\mathcal{W}}_{k}(\mathcal{D})=\left\{\tilde{W}_{j}: 1 \leq j \leq k\right\} \\
\tilde{\mathcal{W}} & =\tilde{\mathcal{W}}(\mathcal{D})=\cup_{k} \tilde{\mathcal{W}}_{k}
\end{aligned}
$$

Definição 2.1.1 Seja $\overline{\mathcal{W}}(\mathcal{D})$ o fecho em $(\Pi, d)$ de $\tilde{\mathcal{W}}(\mathcal{D})$. $\overline{\mathcal{W}}(\mathcal{D})$ é o objeto ao qual chamaremos teia Browniana.

A teia Browniana $\overline{\mathcal{W}}(\mathcal{D})$ possui uma série de propriedades apresentadas na seguinte proposição.

Proposição 2.1.1 $\overline{\mathcal{W}}(\mathcal{D})$ possui as seguintes propriedades.

1. Quase certamente é um subconjunto compacto de $(\Pi, d)$

2. Quase certamente, $\overline{\mathcal{W}}(\mathcal{D})=\lim _{k \rightarrow \infty} \tilde{W}_{k}(\mathcal{D})$, sendo que o limite é tomado em $\mathcal{H}$.

3. A distribuição de $\overline{\mathcal{W}}(\mathcal{D})$ independe de $\mathcal{D}$ e de seu ordenamento.

4. $\overline{\mathcal{W}}(\mathcal{D})$ satisfaz as propriedades 1 e 2 do Teorema 2.1.1; i.e., suas distribuições finito dimensionais correspondem às distribuições de movimentos Brownianos coalescentes. Mais ainda, também satisfaz a propriedade 3 do Teorema 2.1.1.

5. Quase certamente, para todo $\epsilon>0$ e para todo $\theta=\left(f, t_{0}\right) \in \overline{\mathcal{W}}(\mathcal{D})$, existe um caminho $\theta_{\epsilon}=\left(g, t_{0}^{\prime}\right)$ no esqueleto $\tilde{\mathcal{W}}(\mathcal{D})$ tal que $g(s)=f(s)$ para todo $s \geq t_{0}+\epsilon$.

Daremos agora um critério de convergência para variáveis definidas em $\left(\mathcal{H}, \mathcal{F}_{\mathcal{H}}\right)$. Tal critério envolve uma variável aleatória de contagem definida a seguir.

Definição 2.1.2 Dado $t_{0} \in \mathbb{R}, t>0, a<b$ e uma variável aleatória $\left(\mathcal{H}, \mathcal{F}_{\mathcal{H}}\right)$-valuada, $\chi$, seja $\eta_{\chi}\left(t_{0}, t ; a, b\right)$ o número de pontos distintos em $\mathbb{R} \times\left\{t_{0}+t\right\}$ atingidos por caminhos em $\chi$ que também cruzam o segmento $[a, b] \times\left\{t_{0}\right\}$.

Teorema 2.1.2 Suponha que $\mathcal{X}_{1}, \mathcal{X}_{2}, \ldots$ são variáveis aleatórias com valores em $\left(\mathcal{H}, \mathcal{F}_{\mathcal{H}}\right)$ com caminhos que não se cruzam. Se as condições a seguir são válidas, então a distribuição $\mu_{n}$ de $\mathcal{X}_{n}$ converge à distribuição $\mu_{\overline{\mathcal{W}}}$ da teia Browniana standard.

- (I) Para qualquer $y_{1}, y_{2}, \ldots, y_{m} \in \mathcal{D}$ determinísticos, em que $\mathcal{D}$ é um subconjunto (determinístico e arbitrário) denso e enumerável de $\mathbb{R}^{2}$, existem $\theta_{n}^{y_{1}}, \ldots, \theta_{n}^{y_{m}} \in \mathcal{X}_{n}$ tais que $\theta_{n}^{y_{1}}, \ldots, \theta_{n}^{y_{m}}$ convergem em distribuição a movimentos Brownianos coalescentes (com constante de difusão 1) começando em $y_{1}, y_{2}, \ldots, y_{m}$. 
- $\left(B_{1}\right) \forall t>0, \lim \sup _{n \rightarrow \infty} \sup _{\left(a, t_{0}\right) \in \mathbb{R}^{2}} \mu_{n}\left(\eta_{\mathcal{X}_{n}}\left(t_{0}, t ; a, a+\epsilon\right) \geq 2\right) \rightarrow 0$ quando $\epsilon \rightarrow 0+$;

- $\left(B_{2}\right) \forall t>0, \epsilon^{-1} \lim \sup _{n \rightarrow \infty} \sup _{\left(a, t_{0}\right) \in \mathbb{R}^{2}} \mu_{n}\left(\eta_{\mathcal{X}_{n}}\left(t_{0}, t ; a, a+\epsilon\right) \geq 3\right) \rightarrow 0$ quando $\epsilon \rightarrow 0+$

\subsection{Teia Browniana restrita}

Sejam $r, s \in \mathbb{R} \operatorname{com} r<s$ e $A_{r, s}=\mathbb{R} \times[r, s]$. Seja $\bar{A}_{r, s}$ a compatificação de $A_{r, s}$ pela métrica $\bar{\rho}$ dada por:

$$
\bar{\rho}\left(\left(x_{1}, t_{1}\right),\left(x_{2}, t_{2}\right)\right)=\left|\frac{\tanh \left(x_{1}\right)}{1+\left|t_{1}\right|}-\frac{\tanh \left(x_{2}\right)}{1+\left|t_{2}\right|}\right| \vee\left|\tanh \left(t_{1}\right)-\tanh \left(t_{2}\right)\right| .
$$

$\bar{A}_{r, s}$ pode ser pensado como a imagem de $[-\infty, \infty] \times[r, s]$ pela função

$$
(x, t) \rightsquigarrow(\Phi(x, t), \Psi(t)) \equiv\left(\frac{\tanh (x)}{1+|t|}, \tanh (t)\right) .
$$

Para $t_{0} \in[r, s]$ seja $\mathcal{C}_{r, s}\left[t_{0}\right]$ o conjunto das funções $f$ de $\left[t_{0}, s\right]$ em $[-\infty, \infty]$ continuas. Definimos

$$
\Pi_{r, s}=\bigcup_{t_{0} \in[r, s]} \mathcal{C}_{r, s}\left[t_{0}\right] \times\left\{t_{0}\right\}
$$

sendo que $\left(f, t_{0}\right) \in \Pi_{r, s}$ representa um caminho em $\bar{A}_{r, s}$ começando em $\left(f\left(t_{0}\right), t_{0}\right)$ (tal caminho é um ponto no caso $\left.t_{0}=s\right)$. Denotamos por $\hat{f}$ a função que estende $f$ a todo $[r, s]$ definindo $f(t)=f\left(t_{0}\right)$ para $r \leq t \leq t_{0}$. Assim, definimos

$$
d_{r, s}\left(\left(f_{1}, t_{1}\right),\left(f_{2}, t_{2}\right)\right)=\sup _{r \leq t \leq s} \sup _{t}\left|\Phi\left(\hat{f}_{1}(t), t\right)-\Phi\left(\hat{f}_{2}(t), t\right)\right| \vee\left|\Psi\left(t_{1}\right)-\Psi\left(t_{2}\right)\right| .
$$

Nestas condições, $\left(\Pi_{r, s}, d_{r, s}\right)$ é um espaço métrico completo separável.

Seja agora $\mathcal{H}_{r, s}$ o conjunto dos subconjuntos compactos de $\left(\Pi_{r, s}, d_{r, s}\right)$, com $d_{\mathcal{H}_{r, s}}$ a métrica de Hausdorff induzida, i.e.,

$$
d_{\mathcal{H}_{r, s}}\left(K_{1}, K_{2}\right)=\sup _{g_{1} \in K_{1}} \inf _{g_{2} \in K_{2}} d_{r, s}\left(g_{1}, g_{2}\right) \vee \sup _{g_{2} \in K_{2}} \inf _{g_{1} \in K_{1}} d_{r, s}\left(g_{1}, g_{2}\right) .
$$

Temos então que $\left(\mathcal{H}_{r, s}, d_{\mathcal{H}_{r, s}}\right)$ é também um espaço métrico completo separável.

Seja $\mathcal{F}_{\mathcal{H}_{r, s}}$ a $\sigma$-álgebra associada à $d_{\mathcal{H}_{r, s}}$.

A continuação vamos a usar a Teia Browniana para caraterizar e dar criterios de convergência à Teia Browniana restrita. 
Sejam $r, s \in \mathbb{R}$ fixos, com $r<s$. Seja

$$
\hat{\mathcal{H}}_{r}^{s}:=\left\{\mathcal{J} \in \mathcal{H}: \text { Existe }\left(f, t_{0}\right) \in \mathcal{J} \text { com } t_{0} \in[r, s]\right\} .
$$

Isto é, $\hat{\mathcal{H}}_{r}^{s}$ é o subconjunto de $\mathcal{H}$ formado pelas familias de trajetórias compactas que tem pelo menos uma trajetória saindo de $\bar{A}_{r, s}$. É claro que é um conjunto fechado de $\mathcal{H}$, portanto, $\hat{\mathcal{H}}_{r}^{s}$ é um espaço métrico completo separavél.

Consideremos o seguinte mapeamento $\mathfrak{T}$ de $\hat{\mathcal{H}}_{r}^{s}$ em $\mathcal{H}_{r, s}$,

$$
\mathfrak{T}(K)=\left\{\left(f, t_{0}\right) \in K \text { restrita ao conjunto } \bar{A}_{r, s}: t_{0} \in[r, s]\right\}
$$

Isto é, $\mathfrak{T}(K)$ é o conjunto de trajetórias de $K$ que começam em pontos de $\bar{A}_{r, s}$ restritas ao conjunto $\bar{A}_{r, s}$.

Se $K \in \hat{\mathcal{H}}_{r}^{s}$, consideremos o conjunto $K^{\prime}=\left\{\left(f, t_{0}\right) \in K: t_{0} \in[r, s]\right\}$, segui que $K^{\prime}$ é um subconjunto fechado de $K$ e portanto é um subconjunto compacto de trajetórias. Logo, é fácil ver que o conjunto formado pelas trajetórias de $K^{\prime}$ restritas ao conjunto $\bar{A}_{r, s}$ é compacto, isto é, $\mathfrak{T}(K)=\mathfrak{T}\left(K^{\prime}\right)$ é compacto. Portanto $\mathfrak{T}$ esta bem definida.

A continuidade de $\mathfrak{T}$ segue também naturalmente, pois se $\left(K_{n}\right)_{n=1}^{\infty}$ é uma sequência em $\hat{\mathcal{H}}_{r}^{s}$ tal que $K_{n} \underset{n \rightarrow \infty}{\longrightarrow} K$, então claramente $\mathfrak{T}\left(K_{n}\right) \underset{n \rightarrow \infty}{\longrightarrow} \mathfrak{T}(K)$.

Teorema 2.2.1 Existe uma variavél aleatória $\overline{\mathcal{W}}_{r, s}$ com valores em $\left(\mathcal{H}_{r, s}, \mathfrak{F}_{\mathcal{H}_{r, s}}\right)$ cuja distribuição está univocamente determinada pelas três propriedades a seguir:

1. Para qualquer ponto determinístico $(x, t) \in A_{r, s}$ existe, quase certamente, um único caminho $W_{x, t}$ começando em $(x, t)$.

2. Para qualquer número determinístico $n$ e $\left(x_{1}, t_{1}\right), \ldots,\left(x_{n}, t_{n}\right) \in A_{r, s}$ a lei conjunta de $W_{x_{1}, t_{1}}, \ldots, W_{x_{n}, t_{n}}$ é a lei de movimentos Brownianos coalescentes (com constante de difusão unitária).

3. Para qualquer subconjunto denso enumerável $\mathcal{D}$ de $A_{r, s}$, quase certamente, $\overline{\mathcal{W}}_{r, s}$ é o fecho em $\left(\mathcal{H}_{r, s}, \mathfrak{F}_{\mathcal{H}_{r, s}}\right)$ de $\left\{W_{x, t}:(x, t) \in \mathcal{D}\right\}$,

Prova: Do Teorema 2.1.1 temos que $\mathcal{W} \in \hat{\mathcal{H}}_{r}^{s}$ quase certamente, o resultado segue da continuidade de $\mathfrak{T}$ e o Teorema 2.1.1.

Observação 2.2.1 Caso $(x, t)=(x, s)$, então $W_{x, s}$ pode se pensar como um movimento Browniano começando em $(x, s)$ e finalizando em $(x, s)$ (um ponto). 
A variável aleatória $\overline{\mathcal{W}}_{r, s}$ será chamada de Teia Browniana restrita.

O teorema a seguir fornece critérios para verificar a convergência para a Teia Browniana restrita.

Dado $t_{0} \in[r, s], t>0, a<b$ e $\mathcal{M}$ uma variável aleatória assumindo valores em $\left(\mathcal{H}_{r, s}, \mathfrak{F}_{\mathcal{H}_{r, s}}\right)$, seja $\eta_{\mathcal{M}}\left(t_{0}, t ; a, b\right)$ variável aleatória que assume valores em $\{0,1,2, \ldots\}$ representando o número de pontos distintos em $\mathbb{R} \times\left\{t_{0}+t\right\}$ que são alcançados por caminhos de $\mathcal{M}$ que também tocam pontos em $[a, b] \times\left\{t_{0}\right\}$, definimos $\eta_{\mathcal{M}}\left(t_{0}, t ; a, b\right) \equiv 0$ se $t_{0}+t>s$.

Teorema 2.2.2 Suponha que $\mathcal{X}_{1}, \mathcal{X}_{2}, \ldots$ são variáveis aleatórias com valores em $\left(\mathcal{H}_{r, s}, \mathfrak{F}_{\mathcal{H}_{r, s}}\right)$ com caminhos que não se cruzam. Se as condições a seguir são válidas, então a distribuição $\mu_{n}$ de $\mathcal{X}_{n}$ converge à distribuição $\mu_{\mathcal{W}_{r, s}}$ da teia Browniana restrita.

- (I) Para qualquer $y_{1}, y_{2}, \ldots, y_{m} \in \mathcal{D}$ determinísticos, em que $\mathcal{D}$ é um subconjunto (determinístico e arbitrário) denso e enumerável de $A_{r, s}$, existem $\theta_{n}^{y_{1}}, \ldots, \theta_{n}^{y_{m}} \in \mathcal{X}_{n}$ tais que $\theta_{n}^{y_{1}}, \ldots, \theta_{n}^{y_{m}}$ convergem em distribuição a movimentos Brownianos coalescentes (com constante de difusão 1) começando em $y_{1}, y_{2}, \ldots, y_{m}$.

- $\left(B_{1}\right) \forall t>0, \lim \sup _{n \rightarrow \infty} \sup _{\left(a, t_{0}\right) \in A_{r, s}} \mu_{n}\left(\eta \mathcal{X}_{n}\left(t_{0}, t ; a, a+\epsilon\right) \geq 2\right) \rightarrow 0$ quando $\epsilon \rightarrow 0+$;

- $\left(B_{2}\right) \forall t>0, \epsilon^{-1} \lim \sup _{n \rightarrow \infty} \sup _{\left(a, t_{0}\right) \in A_{r, s}} \mu_{n}\left(\eta_{\mathcal{X}_{n}}\left(t_{0}, t ; a, a+\epsilon\right) \geq 3\right) \rightarrow 0$ quando $\epsilon \rightarrow 0+$

Ideia da prova: O fato dos caminhos não se cruzarem, somado a condição (I) garantem que a sequência $\left(\mathcal{X}_{n}\right)_{n=1}^{\infty}$ é justa. As condições $\left(B_{1}\right)$ e $(I)$ garantem que todo limite subsequêncial $\mathcal{X}$ de $\left\{\mathcal{X}_{n}\right\}$ (com distribuição $\mu_{\mathcal{X}}$ ), e para qualquer ponto determinístico $(x, t) \in A_{r, s}$, existe $\mu_{\mathcal{X}}$ quase certamente ao menos um caminho começando de $(x, t)$ (que é um ponto no caso $(x, t)=$ $(x, s))$. Também que a distribuição dos caminhos começando desde subconjuntos finitos de $\mathcal{D}$ são movimentos Brownianos coalescentes (alguns caminhos podem ser simplesmente pontos). Isto mostra que $\mathcal{X}$ contem ao menos os caminhos da Brownian Web restrita $\overline{\mathcal{W}}_{r, s}$.

As condições $\left(B_{1}\right)$ e $\left(B_{2}\right)$ juntas implicam que $\mathbb{E}\left[\eta_{\mathcal{X}}\left(t_{0}, t ; a, b\right)\right] \leq \mathbb{E}\left[\eta_{\overline{\mathcal{W}}}\left(t_{0}, t ; a, b\right)\right]=1+\frac{b-a}{\sqrt{\pi t}}$ para todo $t_{0} \in[r, s], t>0, a, b \in \mathbb{R}$, o qual pelo Teorema 4.6 em [1] implica que $\mathcal{X}$ não contem caminhos extras aqueles de $\overline{\mathcal{W}}_{r, s}$. 


\section{Capítulo 3}

\section{Teia Poissoniana radial discreta}

\subsection{Descrição detalhada do modelo}

Para obter o resultado de convergência fraca da família de curvas de nosso modelo é conveniente realizá-las num espaço apropriado, em que a família limite também esteja contida. Por isto, introduziremos algumas restrições à definição anterior. A primeira se refere ao fato que na escala difusiva que consideraremos, faz sentido incluir apenas caminhos poligonais iniciando numa região angular de ordem $\sqrt{n}$, levando em conta a região radial de ordem $n$. Nossa familia então serão aqueles caminhos poligonais iniciando em pontos de Poisson de um retângulo de lado maior de ordem $n$, ao longo da direção radial e lado menor de ordem $\sqrt{n}$ (de fato um pouco mais do que isto) excluindo a origem. Uma segunda restrição é que é desejável que tais caminhos sejam trajetórias -isto é, que sejam estritamente monotônas na região radial- o que nos fará modificar um pouco a definição de caminhos, restringido-os à região retangular mencionada acima. Esta segunda restrição não introduz uma modificação importante na medida em que os caminhos originais iniciando em pontos do retângulo são trajetórias simultaneamente com alta probabilidade. Chamaremos a familia de curvas desta definição mais precisa de Teia Poissoniana radial discreta (TPRD).

Vamos as definições. Sejam $\alpha \in(0,1)$ e $\delta \in(1 / 4,1 / 3)$ fixos. Seja $z=(x, y) \in \mathbb{R}^{2}-\{(0,0)\}$. Definimos $\theta_{z}$ como sendo igual ao ângulo entre os vetores $\vec{z} \mathrm{e}-\vec{j}=(0,-1)$ e seja $\tilde{\theta}_{z}=\operatorname{sgn}(x) \theta_{z}$. Para $n \in \mathbb{N}$ considere os conjuntos

$$
\mathcal{B}_{n}:=\mathcal{B}_{n}^{\delta, \alpha}:=\left\{z \in \mathbb{R} \times[-n,-n \alpha]:\left|\tilde{\theta}_{z}\right| \leq n^{\delta / 2-1 / 2} / 2\right\}
$$

e

$$
\mathcal{A}_{n}:=\mathcal{A}_{n}^{\delta, \alpha}:=\left\{z \in \mathbb{R} \times[-n,-n \alpha]:\left|\tilde{\theta}_{z}\right| \leq n^{\delta-1 / 2} / 2\right\}
$$


onde $\mathcal{A}_{n}$ é o retângulo que mencionamos acima.

Observação 3.1.1 O fato de ter $\delta \in(0,1 / 3)$ implica que com alta probabilidade, nossa família de curvas poligonais são trajetórias e o fato de ter $\delta>1 / 4$ e $\alpha \in(0,1)$ implica que com alta probabilidade, a família de curvas está contida no int $\mathcal{A}_{n}$, como mostraremos posteriormente.

Para $n \in \mathbb{N}$, sejam $k_{n}=\lfloor n(1-\alpha)\rfloor$ e $A_{k}^{n}:=\left[-\frac{\pi}{2}(n-k), \frac{\pi}{2}(n-k)\right]$ para $k=0,1, \ldots, k_{n}$. Seja $J_{k, n}: A_{k}^{n} \mapsto \tilde{A}_{k}^{n}$, a seguinte função bijetiva $\left(\tilde{A}_{k}^{n}=J_{k, n}\left(A_{k}^{n}\right)\right)$,

$$
J_{k, n}(r)=(n-k)\left(\sin \left(\frac{r}{n-k}\right),-\cos \left(\frac{r}{n-k}\right)\right), \quad 0 \leq k \leq k_{n}+1 .
$$

Observação 3.1.2 Se $r, s \in A_{k}^{n}$ então os pontos $J_{k, n}(r), J_{k, n}(s) \in \tilde{A}_{k}^{n}$ são tais que o comprimento do arco de circunferência em $\tilde{A}_{k}^{n}$ determinado por eles é igual ao comprimento do intervalo determinado por $r, s$ (ver Figura 3.1). Isto é consequência do seguinte, $\tilde{\theta}_{J_{k, n}(r)}=\frac{r}{n-k}, \tilde{\theta}_{J_{k, n}(s)}=$ $\frac{s}{n-k}$, com a norma euclideana $\left\|J_{k, n}(r)\right\|=\left\|J_{k, n}(s)\right\|=n-k$ e lembrando que ângulo $\times$ raio $=$ comprimento do arco, temos que o comprimento do arco determinado por $J_{k, n}(r), J_{k, n}(s)$,

$$
\left|(n-k) \times\left(\frac{r}{n-k}-\frac{s}{n-k}\right)\right|=|r-s| .
$$

Seja $\left(P_{j}\right)_{j=0}^{\infty}$ uma sequência de processos de Poisson independentes de taxa 1 em $\mathbb{R}$. Seja $P_{k}^{n}=$ $P_{k} \cap A_{k}^{n}$, o conjunto de pontos do processo $P_{k}$ sobre $A_{k}^{n}, k=0,1, \ldots, k_{n}+1$ e seja $\hat{P}_{k}^{n}=J_{k, n}\left(P_{k}^{n}\right)$, isto é, os pontos que o processo $P_{k}$ induz sobre $\tilde{A}_{k}^{n}$ pela função $J_{k, n}, k=0,1, \ldots, k_{n}$.

Dizemos que $\hat{P}_{k}^{n}$ é um processo de Poisson de taxa 1 em $\tilde{A}_{k}^{n}$ no sentido da função $J_{k, n}$ sobre $P_{k}^{n}$.

Sejam

$u_{k}^{n}(+)=(n-k)\left(\sin \left(n^{\delta-1 / 2}\right),-\cos \left(n^{\delta-1 / 2}\right)\right) \quad$ e $\quad u_{k}^{n}(-)=(n-k)\left(\sin \left(-n^{\delta-1 / 2}\right),-\cos \left(n^{\delta-1 / 2}\right)\right)$

para $k=0,1, \ldots, k_{n}+1$.

Definição 3.1.1 Seja $\|\cdot\|$ a métrica euclideana em $\mathbb{R}^{2}$. Para $z_{k} \in \tilde{A}_{k}^{n} \cap \mathcal{A}_{n}$, sejam

$$
z_{k}(+):=\arg \min _{\tilde{z} \in \tilde{A}_{k+1}}\left\|z_{k}-\tilde{z}\right\|
$$




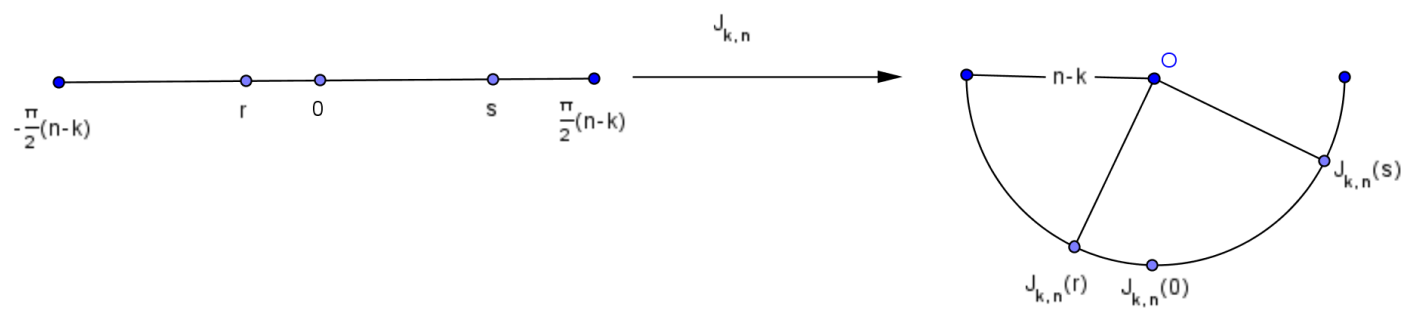

Figura 3.1: $J_{k}^{n}$

$$
\begin{gathered}
\gamma\left(z_{k}\right):=\arg \min _{\tilde{z} \in\left\{u_{k+1}^{n}(+), u_{k+1}^{n}(-)\right\}}\left\|z_{k}-\tilde{z}\right\|, \\
\beta\left(z_{k}\right):=\arg \min _{\tilde{z} \in \hat{P}_{k+1}^{n}}\left\|z_{k}-\tilde{z}\right\| \quad \text { se existir ou } \quad \beta\left(z_{k}\right)=\gamma\left(z_{k}\right) \quad \text { caso contrário }
\end{gathered}
$$

$e$

$$
G_{k}: \tilde{A}_{k}^{n} \times \tilde{A}_{k}^{n} \mapsto[0, \pi(n-k)] \quad \text { onde } \quad G_{k}\left(z_{k}^{1}, z_{k}^{2}\right)=\left|J_{k, n}^{-1}\left(z_{k}^{1}\right)-J_{k, n}^{-1}\left(z_{k}^{2}\right)\right|
$$

para $k=0,1, \ldots, k_{n}$. (Ver Figura 3.2). Note que pela observação 3.1.2 $G_{k}\left(z_{k}^{1}, z_{k}^{2}\right)$ é simplesmente o comprimento do arco de circunferência determinado pelos pontos $z_{k}^{1}, z_{k}^{2}$ em $\tilde{A}_{k}^{n}$.

Note que $\mathbb{P}\left[\arg \min _{\tilde{z} \in \hat{P}_{k+1}^{n}}\left\|z_{k}-\tilde{z}\right\|=\gamma\left(z_{k}\right)\right]=0$, isto é, a probabilidade que um ponto de Poisson esteja sobre a fronteira de $\mathcal{A}_{n}$ é 0 .

Seja $\kappa \in(0,1 / 2-\delta)$ fixo. Dado $z_{k} \in \hat{P}_{k}^{n} \cap \mathcal{B}_{n}$, com $0 \leq k \leq k_{n}$ definimos $\beta^{0}\left(z_{k}\right)=z_{k}$ e 


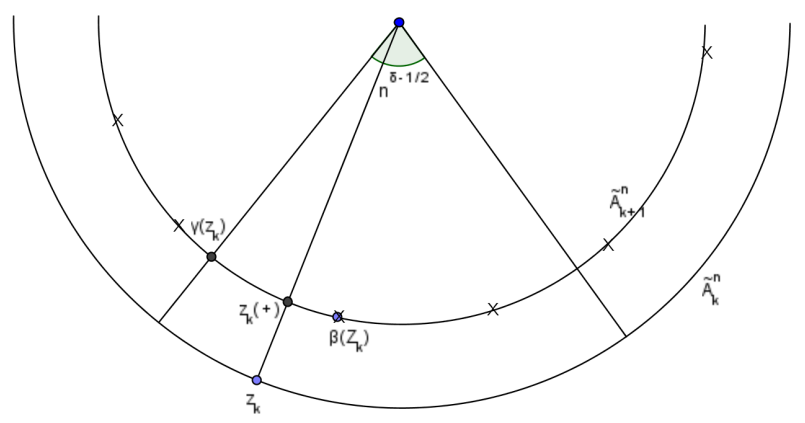

Figura 3.2: Pontos especiais

$\beta^{j}\left(z_{k}\right)=\beta\left(\beta^{j-1}\left(z_{k}\right)\right)$ para $j=1,2, \ldots, k_{n}-k-j$. Seja

$$
J\left(z_{k}\right)=\max \left\{j: \beta^{j}\left(z_{k}\right) \in \mathcal{A}_{n} \cap \hat{P}_{k+j}^{n} \quad \wedge \quad G_{k+j}\left(\beta^{j-1}\left(z_{k}\right)(+), \beta^{j}\left(z_{k}\right)\right) \leq n^{\kappa}\right\} .
$$

se tal $j \in \mathbb{N}$ existir, caso contrario $J\left(z_{k}\right)=0$.

Informalmente, $J\left(z_{k}\right)$ nos diz até qual circunfêrencia acontecem os eventos "bons", isto é, não temos saltos muito "longos" e continuamos interpolando no int $\mathcal{A}_{n}$. Depois de $J\left(z_{k}\right)$ vamos ligar o ultimo ponto à fronteira de $\mathcal{A}_{n}$ de um jeito adequado como veremos agora.

Seja $\xi_{z_{k}}^{n}$ a trajetória obtida por interpolação linear dos pontos

$$
\left\{z_{k}, \beta^{1}\left(z_{k}\right), \ldots, \beta^{J\left(z_{k}\right)}\left(z_{k}\right),(0,0)\right\} \quad \text { se } J\left(z_{k}\right)+k=k_{n}+1
$$

$\mathrm{ou}$

$$
\left\{z_{k}, \beta^{1}\left(z_{k}\right), \ldots, \beta^{J\left(z_{k}\right)}\left(z_{k}\right), \gamma\left(\beta^{J\left(z_{k}\right)}\left(z_{k}\right)\right),(0,0)\right\} \quad \text { se } J\left(z_{k}\right)+k<k_{n}+1
$$

restrita ao conjunto $\mathbb{R} \times[-n,-n \alpha]$.

Considere, 


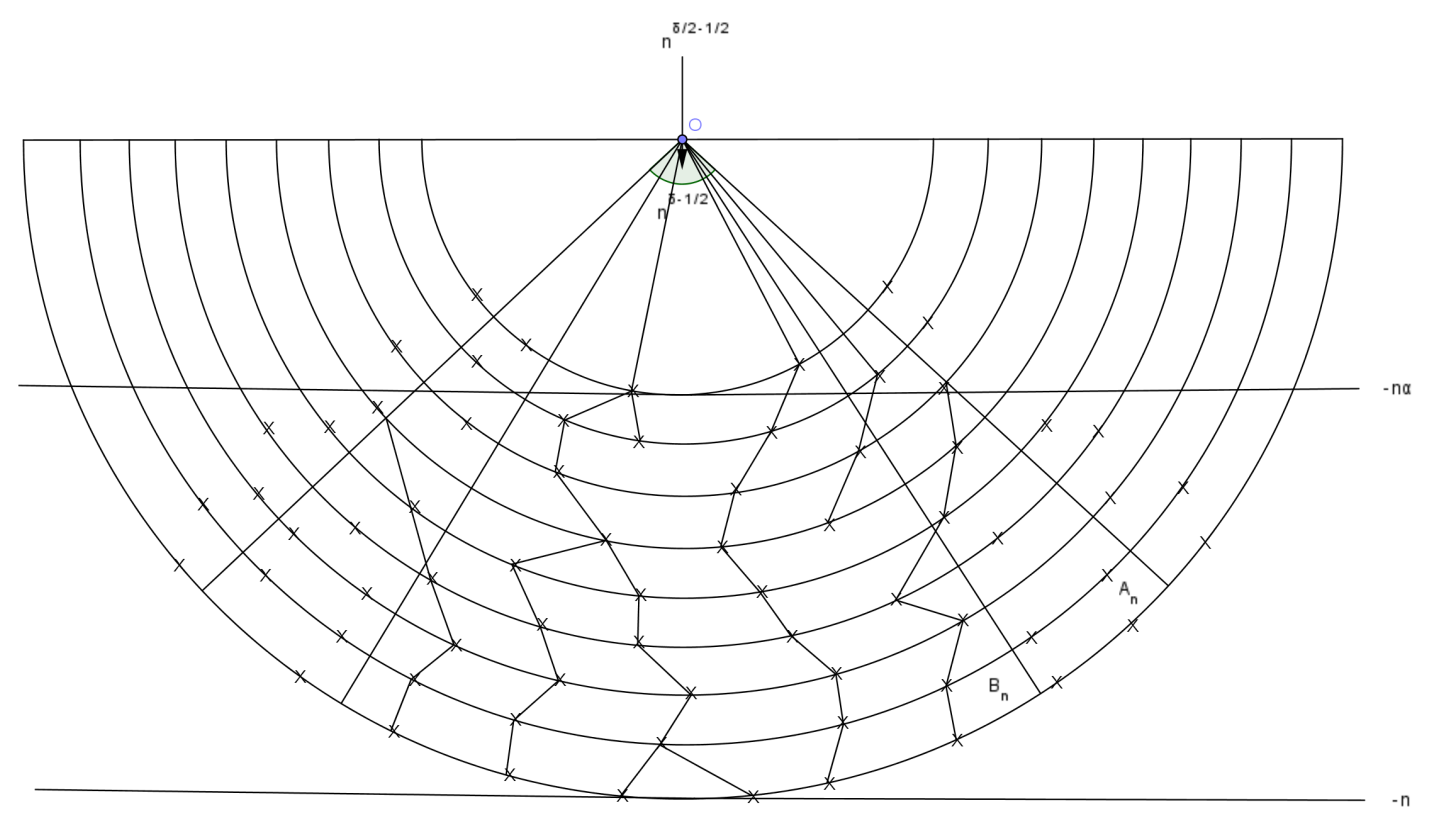

Figura 3.3: Teia Poissoniana radial discreta

$$
\xi^{n}:=\cup_{k=0}^{k_{n}}\left\{\xi_{z_{k}}^{n}: z_{k} \in \hat{P}_{k}^{n} \cap \mathcal{B}_{n}\right\}
$$

o conjunto de todas as trajetórias coalescentes que saem desde $\mathcal{B}_{n}$ restritas ao conjunto $\mathbb{R} \times$ $[-n,-n \alpha]$. Ver Figura 3.3

\subsection{Propriedades da Teia Poissoniana Radial Discreta.}

Apresentaremos as propriedades do conjunto de trajetórias aleatórias $\xi^{n}$ e além disso vamos mostrar porque as modificações feitas à TPRD restritas as trajetórias começando de $\mathcal{B}_{n}$ não são muito importantes.

\section{Propriedades de $\xi^{n}$}

1. $\mathbb{P}\left[\xi^{n}=\emptyset i . v\right]=0$,

2. Existe $N_{0} \in \mathbb{N}$ determinístico tal que $\forall n \geq N_{0}$, $\xi^{n} \in \mathcal{H}_{-n,-n \alpha}$, isto é, $\xi^{n}$ é um conjunto compacto de trajetórias em $\mathbb{R} \times[-n,-n \alpha]$ para todo $n \geq N_{0}$, 
3. $\mathbb{P}\left[\sup _{0 \leq k \leq k_{n}}\left|\hat{P}_{k}^{n} \cap \mathcal{B}_{n}\right|>n i . v\right]=0$,

4. Com alta probabilidade, de cada conjunto $\tilde{A}_{k}^{n} \cap \mathcal{B}_{n}$ sai pelo menos uma trajetória.

5. O evento $\left\{\cup_{k=0}^{k_{n}} \cup_{z_{k} \in \hat{P}_{k}^{n} \cap \mathcal{B}_{n}} \cup_{j=k+1}^{k_{n}+1}\left\{G_{j}\left(\beta^{j-k-1}\left(z_{k}\right)(+), \beta^{j-k}\left(z_{k}\right)\right)>n^{\kappa}\right\}\right\}$ não acontece infinitas vezes e

6. $\mathbb{P}\left[\cup_{k=0}^{k_{n}} \cup_{z \in \hat{P}_{k} \cap \mathcal{B}_{n}} \cup_{j=1}^{k_{n}-k+1}\left\{\beta^{j}(z) \notin i n t \mathcal{A}_{n}\right\}\right.$ i.v $]=0$.

Observação 3.2.1 As propriedades 5 e 6 de $\xi^{n}$ nos dizem precisamente que as modificações feitas não são muito importantes. Também, que com alta probabilidade, as trajetórias de $\xi^{n}$ ficam no interior do conjunto $\mathcal{A}_{n}$.

Prova das propriedades de $\xi^{n}$.

1.

$$
\begin{aligned}
\mathbb{P}\left[\xi^{n}=\emptyset\right] & \leq \sum_{j=0}^{k_{n}} \mathbb{P}\left[\hat{P}_{j}^{n} \cap \mathcal{B}_{n}=\emptyset\right] \\
& \leq \sum_{j=0}^{k_{n}} \mathbb{P}\left[P_{j} \cap\left[-n^{\delta / 2-1 / 2}(n-j), n^{\delta / 2-1 / 2}(n-j)\right]=\emptyset\right] \\
& =\sum_{j=0}^{k_{n}} e^{-2 n^{\delta / 2-1 / 2}(n-j)} \\
& \leq n e^{-2 \alpha n^{\delta / 2+1 / 2}}
\end{aligned}
$$

Pelo Lema de Borel-Cantelli obtemos $\mathbb{P}\left[\begin{array}{ll}\xi^{n}=\emptyset & i . v\end{array}\right]=0$.

2. $\mathcal{H}_{-n,-n \alpha}$ denota o conjunto de subconjuntos compactos de trajetórias em $\mathbb{R} \times[-n,-n \alpha]$ com a métrica definida em 2.17.

É claro que $\emptyset \in \mathcal{H}_{-n,-n \alpha}$, portanto é suficiente mostrar que dado $\xi^{n} \neq \emptyset$ todas as trajetórias aleatórias que pertencem a $\xi^{n}$ estão formadas por interpolação linear de pontos cujas segundas componentes são estritamente crescentes.

Dado $u \in \mathbb{R}^{2}$, denotamos por $u(2)$ a segunda componente de $u$.

Dado $\xi^{n} \neq \emptyset$, seja $\xi_{z_{k}}^{n} \in \xi^{n}$ e suponhamos sem perda de generalidade que $k=0$. Temos que $\xi_{z_{0}}^{n}$ $\left(z_{0} \in \hat{P}_{0}^{n} \cap \mathcal{B}_{n}\right)$ esta formada por interpolação linear dos pontos 


$$
\left\{z_{0}, \beta^{1}\left(z_{0}\right), \ldots, \beta^{J\left(z_{0}\right)}\left(z_{0}\right),(0,0)\right\} \quad \text { se } J\left(z_{0}\right)=k_{n}+1
$$

$\mathrm{Ou}$

$$
\left\{z_{0}, \beta^{1}\left(z_{0}\right), \ldots, \beta^{J\left(z_{0}\right)}\left(z_{0}\right), \gamma\left(\beta^{J\left(z_{0}\right)}\left(z_{0}\right)\right),(0,0)\right\} \quad \text { se } J\left(z_{0}\right)<k_{n}+1
$$

restrita ao conjunto $\mathbb{R} \times[-n,-n \alpha]$.

Dado que $\beta^{J\left(z_{0}\right)}\left(z_{0}\right) \in i n t \mathcal{A}_{n}$ então $\gamma\left(\beta^{J\left(z_{0}\right)}\left(z_{0}\right)\right)$ por definição tem segunda componente estritamente maior que a segunda componente de $\beta^{J\left(z_{0}\right)}\left(z_{0}\right) \in i n t \mathcal{A}_{n}$. Assim, para mostrar que $\xi^{n}$ é uma trajetória é suficiente mostrar que dado $J\left(z_{0}\right)>0$, ocorre que

$$
z_{0}(2)<\beta^{1}\left(z_{0}\right)(2)<\ldots<\beta^{J\left(z_{0}\right)-1}\left(z_{0}\right)(2)<\beta^{J\left(z_{0}\right)}\left(z_{0}\right)(2)
$$

Logo, se $J\left(z_{0}\right)>0$, temos para $j=1, \ldots, J\left(z_{0}\right)$

$$
\beta^{j}\left(z_{0}\right)(2)=-(n-j) \cos \left(\frac{r_{j}}{n-j}\right)
$$

onde $\left|\frac{r_{j}}{n-j}\right|<n^{\delta-1 / 2} / 2$ e além disso $\left|r_{j}-r_{j-1}\right|<n^{\kappa}$.

Assim, para $j=1,2, \ldots, J\left(z_{0}\right)$

$$
\begin{aligned}
\beta^{j}\left(z_{0}\right)(2)-\beta^{j-1}\left(z_{0}\right)(2) & =-(n-j) \cos \left(\frac{r_{j}}{n-j}\right)+(n-j+1) \cos \left(\frac{r_{j-1}}{n-j+1}\right) \\
& =(n-j)\left[\cos \left(\frac{r_{j-1}}{n-j+1}\right)-\cos \left(\frac{r_{j}}{n-j}\right)\right]+\cos \left(\frac{r_{j-1}}{n-j+1}\right)
\end{aligned}
$$

Logo, do Teorema do valor médio,

$$
\beta^{j}\left(z_{0}\right)(2)-\beta^{j-1}\left(z_{0}\right)(2)=(n-j) \sin \left(\alpha_{j}\right)\left(\frac{r_{j-1}}{n-j+1}-\frac{r_{j}}{n-j}\right)+\cos \left(\frac{r_{j-1}}{n-j+1}\right)
$$

para algum $\alpha_{j}$ entre $\frac{r_{j-1}}{n-j+1}$ e $\frac{r_{j}}{n-j}$. Portanto $\exists c_{0}>0$ que não depende de $n, z, j$ tal que 


$$
\begin{aligned}
\left|(n-j) \sin \left(\alpha_{j}\right)\left(\frac{r_{j-1}}{n-j+1}-\frac{r_{j}}{n-j}\right)\right| & \leq c_{0}(n-j)\left|\alpha_{j}\right|\left|\frac{(n-j) r_{j-1}-(n-j+1) r_{j}}{(n-j)(n-j+1)}\right| \\
& =c_{0}\left|\alpha_{j}\right|\left|\frac{(n-j) r_{j-1}-(n-j+1) r_{j}}{(n-j+1)}\right| \\
& =c_{0}\left|\alpha_{j}\right|\left|\frac{(n-j+1) r_{j-1}-(n-j+1) r_{j}-r_{j-1}}{(n-j+1)}\right| \\
& =c_{0}\left|\alpha_{j}\right|\left|r_{j-1}-r_{j}\right|+c_{0}\left|\alpha_{j}\right|\left|\frac{r_{j-1}}{n-j+1}\right| \\
& \leq c_{0} n^{\delta-1 / 2} n^{\kappa}+c_{0} n^{\delta-1 / 2} n^{\delta-1 / 2} \\
& =c_{0} n^{\delta-1 / 2+\kappa}+c_{0} n^{2 \delta-1} .
\end{aligned}
$$

Note que $\delta \in(1 / 4,1 / 3)$ e $\kappa \in(0,1 / 2-\delta)$, seja $N_{0} \in \mathbb{N}$ suficientemente grande tal que $c_{0} n^{\delta-1 / 2+\kappa}+$ $c_{0} n^{2 \delta-1}<1 / 3$ e $\cos \left(n^{\delta-1 / 2}\right) \geq 1 / 2$. Portanto, de (3.11) e (3.12) obtemos que, para $n \geq N_{0}$,

$$
\beta^{j}\left(z_{0}\right)(2)-\beta^{j-1}(z)(2)>0, \quad \text { para } \quad j=1, \ldots, J\left(z_{0}\right) .
$$

Assim, a sequência $\left\{\left(z_{0}\right)(2), \beta^{1}(z)(2), \ldots, \beta^{J\left(z_{0}\right)}\left(z_{0}\right)(2)\right\}$ é estritamente crescente. Portanto, $\forall n \geq$ $N_{0}, \xi_{z_{0}}^{n} \in \mathcal{C}\left[\left(z_{0}\right)_{2},-n \alpha\right]$. Logo, $\forall n \geq N_{0}, \xi^{n} \in \mathcal{H}_{-n,-n \alpha}$.

3.

$$
\begin{aligned}
\mathbb{P}\left[\sup _{0 \leq k \leq k_{n}}\left|\hat{P}_{k}^{n} \cap \mathcal{B}_{n}\right|>n\right] & \leq \sum_{k=0}^{k_{n}} \mathbb{P}\left[\left|\hat{P}_{k}^{n} \cap \mathcal{B}_{n}\right|>n\right] \\
& =\sum_{k=0}^{k_{n}} \mathbb{P}\left[\left|P_{k} \cap\left[-(n-k) n^{\delta / 2-1 / 2},(n-k) n^{\delta / 2-1 / 2}\right]\right|>n\right] \\
& \leq \sum_{k=0}^{k_{n}} \mathbb{P}\left[\left|P_{k} \cap\left[-n^{\delta+1 / 2}, n^{\delta+1 / 2}\right]\right|>n\right] \\
& =\sum_{k=0}^{k_{n}} \mathbb{P}\left[\left|P_{0} \cap\left[-n^{\delta+1 / 2}, n^{\delta+1 / 2}\right]\right|>n\right]
\end{aligned}
$$

Seja $N_{n}=\left\{\left|P_{0} \cap\left[-n^{\delta+1 / 2}, n^{\delta+1 / 2}\right]\right|>n\right\}$, então 


$$
\begin{aligned}
\mathbb{P}\left[N_{n}\right] & =\mathbb{P}\left[\left|P_{0} \cap\left[-n^{\delta+1 / 2}, n^{\delta+1 / 2}\right]\right|>n\right] \\
& =\mathbb{P}\left[\sum_{i=1}^{n} T_{i}<2 n^{\delta+1 / 2}\right]
\end{aligned}
$$

com $\left(T_{i}\right)_{1 \leq i \leq n}$ variáveis aleatórias i.i.d exponenciais de taxa 1. Portanto, $M_{n}=\sum_{i=1}^{n} T_{i}$ tem distribuição Gamma de parâmetros $n$ e 1. Isto é, tem função de densidade de probabilidade

$$
f_{M_{n}}(t)=e^{-t} \frac{t^{n-1}}{(n-1) !}, \quad t \geq 0
$$

Assím,

$$
\begin{aligned}
\mathbb{P}\left[\sum_{i=1}^{n} T_{i}<2 n^{\delta+1 / 2}\right] & =\int_{0}^{2 n^{\delta+1 / 2}} e^{-t} \frac{t^{n-1}}{(n-1) !} d t \\
& \leq \frac{\left(2 n^{\delta+1 / 2}\right)^{(n-1)}}{(n-1) !} \int_{0}^{2 n^{\delta+1 / 2}} e^{-t} d t \\
& \leq \frac{\left(2 n^{\delta+1 / 2}\right)^{(n-1)}}{(n-1) !}
\end{aligned}
$$

Da aproximação de Stirling $(n-1) ! \approx \sqrt{2 \pi(n-1)}\left(\frac{n-1}{e}\right)^{n-1}$, obtemos para $n$ suficientemente grande

$$
\mathbb{P}\left[\sum_{i=1}^{n} T_{i}<2 n^{\delta+1 / 2}\right] \leq c_{0} e^{-c_{1} n} .
$$

onde $c_{0}, c_{1}$ são constantes positivas que não dependem de $n$.

Logo, de (3.13), (3.14) e (3.15) obtemos,

$$
\mathbb{P}\left[\sup _{0 \leq k \leq k_{n}}\left|\hat{P}_{k}^{n} \cap \mathcal{B}_{n}\right| \geq n\right] \leq c_{0} n e^{-c_{1} n}
$$

Pelo Lema de Borel-Cantelli $\mathbb{P}\left[\sup _{0 \leq k \leq k_{n}}\left|\hat{P}_{k}^{n} \cap \mathcal{B}_{n}\right| \geq n \quad i . v\right]=0$. 
4.

$$
\begin{aligned}
\mathbb{P}\left[\min _{1 \leq j \leq k_{n}}\left|\hat{P}_{j}^{n} \cap \mathcal{B}_{n}\right|=0\right] & \leq \sum_{j=1}^{k_{n}} \mathbb{P}\left[\left|\hat{P}_{j}^{n} \cap \mathcal{B}_{n}\right|=0\right] \\
& \leq \sum_{j=1}^{k_{n}} e^{-2(n-j) n^{\delta / 2-1 / 2}} \\
& \leq \sum_{j=1}^{k_{n}} e^{-2 \alpha n^{\delta / 2+1 / 2}} \\
& \leq n e^{-2 \alpha n^{\delta / 2+1 / 2}}
\end{aligned}
$$

Portanto, com alta probabilidade de cada $\left(\tilde{A}_{k}^{n}\right)$ sai pelo menos uma trajetória e com alta probabilidade para cada ponto de saída $z_{k} \in \hat{P}_{k}^{n} \cap \mathcal{B}_{n}, \beta^{j}\left(z_{k}\right) \in \hat{P}_{k}^{n}, j=0,1, \ldots, k_{n}+1-k$.

5. Sejam $U_{n}:=\left\{\cup_{k=0}^{k_{n}} \cup_{z \in \hat{P}_{k} \cap \mathcal{B}_{n}} \cup_{j=k+1}^{k_{n}+1}\left\{G_{j}\left(\beta^{j-k-1}(z)(+), \beta^{j-k}(z)\right)>n^{\kappa}\right\}\right\} \mathrm{e}$

$$
\left.D_{n}^{k}:=\left\{\cup_{z \in \hat{P}_{k} \cap \mathcal{B}_{n}} \cup_{j=k}^{k_{n}+1}\left\{G_{j}\left(\beta^{j-k-1}(z)(+), \beta^{j-k}(z)\right)>n^{\kappa}\right)\right\}\right\} \quad \text { para } \quad k=0,1, \ldots, k_{n} .
$$

Assim,

$$
\mathbb{P}\left[U_{n}\right] \leq \sum_{k=0}^{k_{n}} \mathbb{P}\left[D_{n}^{k}\right] .
$$

Logo, seja $V_{n}:=\left\{\left|\hat{P}_{0}^{n} \cap \mathcal{B}_{n}\right| \leq n\right\} \cap\left\{\hat{P}_{k}^{n} \neq \emptyset, k=0,1, \ldots, k_{n}\right\}$. Então

$$
\begin{aligned}
\mathbb{P}\left[D_{n}^{0}\right] & =\mathbb{P}\left[D_{n}^{0} \mid V_{n}\right] \mathbb{P}\left[V_{n}\right]+\mathbb{P}\left[D_{n}^{0} \mid V_{n}^{c}\right] \mathbb{P}\left[V_{n}^{c}\right] \\
& \leq \mathbb{P}\left[D_{n}^{0} \mid V_{n}\right]+\mathbb{P}\left[V_{n}^{c}\right]
\end{aligned}
$$

Agora, $\mathbb{P}\left[D_{n}^{0} \mid V_{n}\right] \leq n^{2} k_{n} e^{-n^{\kappa}} \leq n^{3} e^{-n^{\kappa}}$ e da Propriedade 3 de $\xi^{n}$, segue

$$
\begin{aligned}
\mathbb{P}\left[V_{n}^{c}\right] & \leq \mathbb{P}\left[\left|\hat{P}_{0}^{n} \cap \mathcal{B}_{n}\right|>n\right]+\mathbb{P}\left[\exists k, 0 \leq k \leq k_{n}: \hat{P}_{k}^{n}=\emptyset\right] \\
& \leq c_{0} e^{-c_{1} n}+\sum_{j=0}^{k_{n}} \mathbb{P}\left[\hat{P}_{k}^{n}=\emptyset\right] \\
& \leq c_{0} e^{-c_{1} n}+n e^{-2 \alpha n}
\end{aligned}
$$


Logo, $\mathbb{P}\left[D_{n}^{0}\right] \leq c_{2} n^{3} e^{-n^{\kappa}}, c_{2}>0$.

Analogamente se prova que,

$$
\mathbb{P}\left[D_{n}^{k}\right] \leq c_{2} n^{3} e^{-n^{\kappa}} \quad \text { para } \quad k=0,1, \ldots, k_{n} .
$$

Logo, de (3.17) e (3.18), obtemos que

$$
\mathbb{P}\left[U_{n}\right] \leq c_{2} n^{4} e^{-n^{\kappa}}
$$

O resultado segue do Lema de Borel-Cantelli.

6. Sejam $R_{n}:=\left\{\cup_{k=0}^{k_{n}-1} \cup_{z_{k} \in \hat{P}_{k} \cap \mathcal{B}_{n}} \cup_{j=k+1}^{k_{n}}\left\{\beta^{j}\left(z_{k}\right) \notin i n t \mathcal{A}_{n}\right\}\right\}$ e

$$
M_{n}^{k}:=\left\{\cup_{z_{k} \in \hat{P}_{k} \cap \mathcal{B}_{n}} \cup_{j=k+1}^{k_{n}}\left\{\beta^{j}\left(z_{k}\right) \notin i n t \mathcal{A}_{n}\right\}\right\}
$$

para $k=0,1, \ldots, k_{n}-1$.

Logo, seja $W_{n}:=\left\{0<\left|\hat{P}_{0}^{n} \cap \mathcal{B}_{n}\right| \leq n\right\}$. Temos que

$$
\begin{aligned}
\mathbb{P}\left[M_{n}^{0}\right] & =\mathbb{P}\left[M_{n}^{0} \mid W_{n}\right] \mathbb{P}\left[W_{n}\right]+\mathbb{P}\left[M_{n}^{0} \mid W_{n}^{c}\right] \mathbb{P}\left[W_{n}^{c}\right] \\
& \leq \mathbb{P}\left[M_{n}^{0} \mid W_{n}\right]+\mathbb{P}\left[W_{n}^{c}\right]
\end{aligned}
$$

Dado $z_{k} \in \hat{P}_{k}^{n} \cap \mathcal{A}_{n}$ e lembrando que $\hat{P}_{k}^{n}=J_{k, n}\left(P_{k}^{n}\right)$ concluimos que $z_{k}$ pode ser representado da seguinte forma:

$$
z_{k}=(n-k)\left(\sin \left(\frac{\varepsilon_{z_{k}}}{n-k}\right),-\cos \left(\frac{\varepsilon_{z_{k}}}{n-k}\right)\right)
$$

onde $\varepsilon_{z_{k}} \in P_{k}^{n}$ e $\left|\frac{\varepsilon_{z_{k}}}{n-k}\right| \leq n^{\delta-1 / 2} / 2$.

Dado $z_{0} \in \hat{P}_{0}^{n} \cap \mathcal{B}_{n}$, seja $b_{0}\left(z_{0}\right)=\tilde{\theta}_{z_{0}}$. Definimos indutivamente

$$
b_{j}\left(z_{0}\right)=\frac{\arg \min _{x \in P_{j}}\left|b_{j-1}\left(z_{0}\right)-\frac{x}{n-j}\right|}{n-j}, \quad j=1,2, \ldots, k_{n}+1
$$

Note que dado $b_{j-1}\left(z_{0}\right), b_{j}\left(z_{0}\right)-b_{j-1}\left(z_{0}\right) \stackrel{\mathcal{D}}{=} \frac{\arg \min _{x \in P_{j}}}{n-j}$. Seja $\varsigma_{j}\left(z_{0}\right)=b_{j}\left(z_{0}\right)-b_{j-1}\left(z_{0}\right)$, $j=1,2, \ldots, k_{n}+1$.

Logo, o evento $\left\{\beta^{j}\left(z_{0}\right) \notin i n t \mathcal{A}_{n}\right.$ para algum $\left.j, 1 \leq j \leq k_{n}+1\right\}$ esta contido no evento 


$$
\left\{\sup _{1 \leq j \leq k_{n}+1}\left|b_{0}\left(z_{0}\right)+\sum_{i=1}^{j} \varsigma_{i}\left(z_{0}\right)\right|>n^{\delta-1 / 2} / 2\right\} \cup D_{n}^{0}
$$

onde $\left.D_{n}^{0}=\left\{\cup_{z_{0} \in \hat{P}_{0}^{n} \cap \mathcal{B}_{n}} \cup_{j=1}^{k_{n}+1}\left\{G_{j}\left(\beta^{j-1}\left(z_{0}\right)(+), \beta^{j}\left(z_{0}\right)\right)>n^{\kappa}\right)\right\}\right\}$.

Agora, como $\left|b_{0}\left(z_{0}\right)\right|=\left|\tilde{\theta}_{z_{0}}\right|<n^{\delta / 2-1 / 2} / 2$. Temos que para $n$ suficientemente grande

$$
\left\{\sup _{1 \leq j \leq k_{n}+1}\left|b_{0}\left(z_{0}\right)+\sum_{i=1}^{j} \varsigma_{i}\left(z_{0}\right)\right|>n^{\delta-1 / 2} / 2\right\} \subset\left\{\sup _{1 \leq j \leq k_{n}+1}\left|\sum_{i=1}^{j} \varsigma_{i}\left(z_{0}\right)\right|>\frac{n^{\delta-1 / 2}}{4}\right\} .
$$

Seja $\varepsilon_{j}=\arg \min _{x \in P_{j}}|x|, j=1, \ldots, k_{n}+1$. Assim,

$$
\begin{aligned}
\mathbb{P}\left[M_{n}^{0} \mid W_{n}\right] & \leq n \mathbb{P}\left[\sup _{1 \leq j \leq k_{n}+1}\left|\sum_{i=1}^{j} \frac{\varepsilon_{i}}{n-i}\right|>\frac{n^{\delta-1 / 2}}{4}\right]+n \mathbb{P}\left[D_{n}^{0}\right] \\
& \leq n \sum_{j=1}^{k_{n}+1} \mathbb{P}\left[\left|\sum_{i=1}^{j} \frac{\varepsilon_{i}}{n-i}\right|>\frac{n^{\delta-1 / 2}}{4}\right]+n \mathbb{P}\left[D_{n}^{0}\right] \\
& \leq n \sum_{j=1}^{\lfloor\sqrt{n}\rfloor} \mathbb{P}\left[\left|\sum_{i=1}^{j} \frac{\varepsilon_{i}}{n-i}\right|>\frac{n^{\delta-1 / 2}}{4}\right]+n \sum_{j=\lfloor\sqrt{n}\rfloor+1}^{k_{n}+1} \mathbb{P}\left[\left|\sum_{i=1}^{j} \frac{\varepsilon_{i}}{n-i}\right|>\frac{n^{\delta-1 / 2}}{4}\right]+n \mathbb{P}\left[D_{n}^{0}\right] \\
& \leq n\lfloor\sqrt{n}\rfloor \times\lfloor\sqrt{n}\rfloor e^{-\frac{n-\lfloor\sqrt{n}\rfloor}{\lfloor\sqrt{n}\rfloor} \frac{n^{\delta-1 / 2}}{4}}+n \sum_{j=\lfloor\sqrt{n}\rfloor+1}^{k_{n}+1} \mathbb{P}\left[\left|\sum_{i=1}^{j} \frac{\varepsilon_{i}}{n-i}\right|>\frac{n^{\delta-1 / 2}}{4}\right]+n \mathbb{P}\left[D_{n}^{0}\right] \\
& \leq n\lfloor\sqrt{n}\rfloor \times\lfloor\sqrt{n}\rfloor e^{-\frac{n-\lfloor\sqrt{n}\rfloor}{\lfloor\sqrt{n}\rfloor} \frac{n^{\delta-1 / 2}}{4}+n} \sum_{j=\lfloor\sqrt{n}\rfloor+1}^{k_{n}+1} \mathbb{P}\left[\left|\sum_{i=1}^{j} \frac{n \varepsilon_{i}}{n-i}\right|>\frac{n \times n^{\delta-1 / 2}}{4}\right]+n \mathbb{P}\left[D_{n}^{0}\right] \\
& \leq n^{2} e^{-c_{0} n^{\delta}}+n \sum_{j=\lfloor\sqrt{n}\rfloor+1}^{k_{n}+1} e^{-c_{1} j\left(\frac{n^{\delta-1 / 2}}{4}\right)^{2}}+n \mathbb{P}\left[D_{n}^{0}\right] \quad\left(c_{0}, c_{1}>0\right) .
\end{aligned}
$$

A última desigualdade é consequência da desigualdade de Bernstein para Martingais, onde $\left(\frac{n}{n-i} \varepsilon_{i}\right)_{i=0}^{k_{n}}$ é a sequência Martingal em relação à filtração $\mathcal{F}_{i}=\sigma\left(\varepsilon_{0}, \varepsilon_{1}, \ldots, \varepsilon_{i}\right)$ e de $(3.18)$, obtemos

$$
\mathbb{P}\left[M_{n}^{0} \mid W_{n}\right] \leq n^{2} e^{-c_{0} n^{\delta}}+n^{2} e^{-c_{2} n^{2 \delta-1 / 2}}+c_{3} n^{4} e^{-n^{\kappa}}, \quad \delta \in(1 / 4,1 / 3)
$$

De (3.19) e (3.23), obtemos

$$
\mathbb{P}\left[M_{n}^{0}\right] \leq n^{2} e^{-c_{0} n^{\delta}}+n^{2} e^{-c_{2} n^{2 \delta-1 / 2}}+c_{3} n^{4} e^{-n^{\kappa}}+\mathbb{P}\left[W_{n}^{c}\right]
$$

Além disso, $\mathbb{P}\left[W_{n}^{c}\right]=\mathbb{P}\left[\left|\hat{P}_{0}^{n} \cap \mathcal{B}_{n}\right|=0\right]+\mathbb{P}\left[\left|\hat{P}_{0}^{n} \cap \mathcal{B}_{n}\right|>n\right]$. Da propriedade 3 de $\xi^{n}$ obtemos 


$$
\mathbb{P}\left[W_{n}^{c}\right] \leq e^{-n^{\delta+1 / 2}}+c_{4} e^{-c_{5} n} \leq c_{6} e^{-n^{\delta+1 / 2}} .
$$

Portanto de (3.24),

$$
\mathbb{P}\left[M_{n}^{0}\right] \leq n^{2} e^{-c_{0} n^{\delta}}+n^{2} e^{-c_{2} n^{2 \delta-1 / 2}}+c_{3} n^{4} e^{-n^{\kappa}}+c_{6} e^{-n^{\delta+1 / 2}} \leq c_{7} n^{4} e^{-n^{\kappa}} \quad\left(c_{7}>0\right) .
$$

Analogamente, mostramos que

$$
\mathbb{P}\left[M_{n}^{k}\right] \leq c_{7} n^{4} e^{-n^{\kappa}}, \quad k=0,1, \ldots, k_{n}-1
$$

Logo,

$$
\mathbb{P}\left[R_{n}\right] \leq \sum_{k=0}^{k_{n}-1} \mathbb{P}\left[M_{n}^{k}\right] \leq c_{7} n^{5} e^{-n^{\kappa}} .
$$

Por fim, do Lema de Borel-Cantelli segue que

$$
\mathbb{P}\left[R_{n} \quad \text { i.v }\right]=0 .
$$

como queriamos demonstrar.

\subsection{Enunciado do Teorema principal}

O principal resultado neste trabalho é mostrar que $\xi^{n}$ na escala difusiva converge fracamente para um mapeamento continuo da Teia Browniana restrita $(\mathrm{em} \mathbb{R} \times[0,1 / \alpha-1])$.

Sejam $F_{\alpha}:=\mathbb{R} \times[-1,-\alpha]$ e $G_{\alpha}:=\mathbb{R} \times[0,1 / \alpha-1]$. Seja $\psi: F_{\alpha} \mapsto G_{\alpha}$ a seguinte homeomorfismo

$$
\psi(x, t)=\left(\frac{x}{|t|}, \frac{1}{|t|}-1\right)
$$

Assim

$$
\psi^{-1}\left(x^{\prime}, t^{\prime}\right)=\left(\frac{x^{\prime}}{t^{\prime}+1},-\frac{1}{t^{\prime}+1}\right) .
$$

Para $r, s \in \mathbb{R}, r<s, \mathcal{H}_{r, s}$ representa o conjunto formado por subconjuntos compactos de trajetórias que saem de pontos em $\mathbb{R} \times[r, s]$ restritos ao mesmo conjunto com a métrica definida em (2.17).

Seja $T: \mathcal{H}_{-1,-\alpha} \longrightarrow \mathcal{H}_{0,1 / \alpha-1}$ definida por 


$$
T(\mathcal{G})=\{(\psi(f(t), t))\}_{(f(t), t) \in \mathcal{G}}
$$

e seja

$$
T^{-1}(\mathcal{F})=\left\{\left(\psi^{-1}(f(t), t)\right)\right\}_{(f(t), t) \in \mathcal{F}} .
$$

Para $\mathcal{F} \in \mathcal{H}_{-n,-n \alpha}$, definimos

$$
\mathfrak{E}^{n}[\mathcal{F}]=\left\{\left(\frac{f(t)}{\sqrt{n}}, \frac{t}{n}\right):(f(t), t) \in \mathcal{F}\right\}
$$

Note que $\mathfrak{E}^{n}[\mathcal{F}] \in \mathcal{H}_{-1,-\alpha}$.

O resultado principal deste trabalho é a convergência fraca na escala difusiva de $\xi^{n}$ para um mapeamento continuo da Teia Browniana restrita (ver 2.2) em $\mathbb{R} \times[0,1 / \alpha-1]$. Segue o enunciado formal do Teorema principal.

Teorema 3.3.1 Seja $N_{0} \in \mathbb{N}$ como na Propriedade 2 de $\xi_{n}$, considere a sequência $\left(\xi^{n}\right)_{n \geq N_{0}}$ em $\mathcal{H}_{-n,-n \alpha}$. Então, $\left(\xi^{\mathfrak{n}}\right)_{n \geq N_{0}}$ é uma sequência em $\mathcal{H}_{-1,-\alpha}$ e

$$
\mathfrak{E}^{n}\left[\xi^{n}\right] \underset{n \rightarrow \infty}{\stackrel{\mathcal{D}}{\longrightarrow}} T^{-1}\left(\overline{\mathcal{W}}_{0,1 / \alpha-1}\right)
$$




\section{Capítulo 4}

\section{Aproximações do modelo}

Seja $\tilde{\xi}^{n}=\mathfrak{E}^{n}\left[\xi^{n}\right]$, para mostrar o Teorema 3.3.1, é necessário construir um conjunto de "aproximações" a $\tilde{\xi}^{n}$. As ideias de cada aproximação, a grosso modo, são:

- para a primeira aproximação, fazemos uma projeção adequada de todos os pontos de Poisson de cada trajetória de $\xi^{n}$, identificamos os pontos projetados de cada trajetória e construimos uma nova trajetória dada pela interpolação linear de tais pontos, assim, temos uma nova família de trajetórias com a propriedade desejada.

- a ideia para a segunda aproximação é que as modificações feitas à TPRD não acontecem com alta probabilidade, nesse sentido pode-se dar "liberdade" ao comportamento do ângulo

- e finalmente a ideia para a terceira aproximação é que $\sin (x) \approx x$ para $x$ suficientemente pequeno.

\subsection{Primeira aproximação.}

Seja $z_{k} \in \hat{P}_{k}^{n} \cap \mathcal{A}_{n}$, para $0 \leq k \leq k_{n}+1$, definimos:

$$
h\left(z_{k}\right):=(n-k)\left(\sin \left(\frac{\varepsilon_{z_{k}}}{n-k}\right),-1\right) .
$$

Isto é, $h\left(z_{k}\right)$ é a projeção do ponto $z_{k}$ sobre a linha reta $\mathbb{R} \times\{n-k\}$.

Sejam

$$
\begin{gathered}
\Gamma_{n}:=\Gamma_{n}^{\delta, \alpha}:=\left\{z: z \in \hat{P}_{k}^{n} \quad \text { e } \quad\left|\theta_{z}\right| \leq n^{\delta-1 / 2} / 2 \quad \text { para algum } \quad k, 0 \leq k \leq k_{n}+1\right\} \quad \text { e } \\
\tilde{\Gamma}_{n}:=\tilde{\Gamma}_{n}^{\delta, \alpha}:=\left\{h(z): z \in \Gamma_{n}\right\} .
\end{gathered}
$$


Seja $\rho$ é a métrica de Hausdorff induzida pelos subconjuntos compactos de $\mathbb{R}^{2}$. Definimos $\rho(\emptyset, \emptyset)=0$.

Definição 4.1.1 Para $A \subset \mathbb{R}^{2}$, seja $E^{n}(A):=\left\{\left(\frac{x}{\sqrt{n}}, \frac{y}{n}\right):(x, y) \in A\right\}$.

Lema 4.1.1 $\rho\left(E^{n}\left(\Gamma_{n}\right), E^{n}\left(\tilde{\Gamma}_{n}\right)\right) \leq 1-\cos \left(n^{\delta-1 / 2} / 2\right)$

Prova: Se $\Gamma_{n}=\emptyset$ então $E^{n}\left(\Gamma_{n}\right)=E^{n}\left(\tilde{\Gamma}_{n}\right)=\emptyset \operatorname{assim} \rho\left(E^{n}\left(\Gamma_{n}\right), E^{n}\left(\tilde{\Gamma}_{n}\right)\right)=0$.

Se $\Gamma_{n} \neq \emptyset$, seja $z \in \Gamma_{n}, \operatorname{logo}$

$$
z=(n-k)\left(\sin \left(\frac{\epsilon_{z}}{n-k}\right),-\cos \left(\frac{\epsilon_{z}}{n-k}\right)\right) \quad \text { para algum } k \leq k_{n},
$$

onde $\epsilon_{z} \in \tilde{P}_{k}^{n}$ e $\left|\frac{\epsilon_{z}}{n-k}\right| \leq n^{\delta-1 / 2} / 2$. Portanto,

$$
h(z)=(n-k)\left(\sin \left(\frac{\epsilon_{z}}{n-k}\right),-1\right) .
$$

Assím,

$$
\left\|E^{n}(\{z\})-E^{n}(\{h(z)\})\right\|=\frac{n-k}{n}\left[1-\cos \left(\frac{\epsilon_{z}}{n-k}\right)\right] \leq 1-\cos \left(n^{\delta-1 / 2} / 2\right) .
$$

Dado $z_{k} \in \hat{P}_{k}^{n} \cap \mathcal{B}_{n}$, lembremos que $\tilde{\xi}_{z_{k}}^{n}$ é a trajetória obtida por interpolação linear dos pontos

$$
\left\{z_{k}, \beta^{1}\left(z_{k}\right), \ldots, \beta^{J\left(z_{k}\right)}\left(z_{k}\right),(0,0)\right\} \quad \text { se } \quad J\left(z_{k}\right)+k=k_{n}+1
$$

ou

$$
\left\{z_{k}, \beta^{1}\left(z_{k}\right), \ldots, \beta^{J\left(z_{k}\right)}\left(z_{k}\right), \gamma\left(\beta^{J\left(z_{k}\right)}\left(z_{k}\right)\right)(0,0)\right\} \quad \text { se } \quad J\left(z_{k}\right)+k<k_{n}+1
$$

restrita ao conjunto $\mathbb{R} \times[-n,-n \alpha]$.

Definimos $\xi_{z_{k}}^{1, n}$ como a trajetória obtida de interpolar linearmente os pontos

$$
\left\{h\left(z_{k}\right), h\left(\beta^{1}\left(z_{k}\right)\right), \ldots, h\left(\beta^{J\left(z_{k}\right)}\left(z_{k}\right)\right),(0,0)\right\} \quad \text { se } \quad J\left(z_{k}\right)+k=k_{n}+1
$$

ou 


$$
\left\{h\left(z_{k}\right), h\left(\beta^{1}\left(z_{k}\right)\right), \ldots, h\left(\beta^{J\left(z_{k}\right)}\left(z_{k}\right)\right), h\left(\gamma\left(\beta^{J\left(z_{k}\right)}\left(z_{k}\right)\right)\right),(0,0)\right\} \quad \text { se } \quad J\left(z_{k}\right)+k<k_{n}+1
$$

restrita ao conjunto $\mathbb{R} \times[-n,-n \alpha]$.

Definimos,

$$
\xi^{1, n}:=\left\{\xi_{z_{k}}^{1, n}: z_{k} \in \hat{P}_{k} \cap \mathcal{B}_{n}\right\}_{0 \leq k \leq k_{n}} .
$$

Isto é, $\xi^{1, n}$ é o conjunto de trajetórias coalescentes onde cada trajetória é identificada com exatamente uma trajetória de $\xi^{n}$ restritas ao conjunto $\mathbb{R} \times[-n,-n \alpha]$.

Sejam

$$
\tilde{\xi}^{n}:=\mathfrak{E}^{n}\left(\xi^{n}\right) \quad \text { e } \quad \tilde{\xi}^{1, n}:=\mathfrak{E}^{n}\left(\xi^{1, n}\right)
$$

isto é, as correspondentes familias de trajetórias aleatórias coalescentes re-escaladas.

Lema 4.1.2 Existe $N_{0} \in \mathbb{N}$ determinístico tal que para todo $n \geq N_{0}$ temos que $\tilde{\xi}^{n}, \tilde{\xi}^{1, n} \in \mathcal{H}_{-1,-\alpha}$.

Prova: $\tilde{\xi}^{1, n} \in \mathcal{H}_{-1,-\alpha}$ para todo $n \in \mathbb{N}$ por construção e pela propriedade 2 de $\xi^{n}$, existe um $N_{0} \in \mathbb{N}$ determinístico tal que $\tilde{\xi} \in \mathcal{H}_{-1,-\alpha}$ para todo $n \geq N_{0}$.

Lema 4.1.3 $d_{\mathcal{H}_{-1,-\alpha}}\left(\tilde{\xi}^{n}, \tilde{\xi}^{1, n}\right) \underset{n \rightarrow \infty}{\stackrel{q . c}{\rightarrow}} 0$.

Prova: Segui da observação 3.2.1, do fato que a interpolação dos pontos é de tipo linear e o Lema 4.1.1.

Lembrando que nosso objetivo é encontrar o limite fraco de $\tilde{\xi}^{n}$, do lema anterior temos que é suficiente conhecer o limite fraco de $\tilde{\xi}^{1, n}$. 


\subsection{Segunda aproximação}

Agora nosso objetivo é encontrar o limite fraco de $\tilde{\xi}^{1, n}$, com isso em mente, vamos a construir um conjunto de trajetórias aleatórias coalescentes que chamaremos $\tilde{\xi}^{2, n}$ com a propriedade que a distância entre ela e $\tilde{\xi}^{1, n}$ em $\mathcal{H}_{-1,-\alpha}$ vai para zero quase certamente e portanto elas terão o mesmo limite fraco.

Sejam

$$
\begin{gathered}
A_{n}:=\left\{\min _{1 \leq j \leq k_{n}}\left|\hat{P}_{j}^{n} \cap \mathcal{B}_{n}\right|>0\right\}, \\
B_{n}:=\left\{\cup_{k=0}^{k_{n}} \cup_{z \in \hat{P}_{k}^{n} \cap \mathcal{B}_{n}} \cup_{j=k+1}^{k_{n}}\left\{G_{j}\left(\beta^{j-1}(z)(+), \beta^{j}(z)\right)>n^{\kappa}\right\}\right\}^{c}, \\
C_{n}:=\left\{\cup_{k=0}^{k_{n}-1} \cup_{z \in \hat{P}_{k}^{n} \cap \mathcal{B}_{n}} \cup_{j=k+1}^{k_{n}}\left\{\beta^{j}(z) \notin \mathcal{A}_{n}\right\}\right\}^{c}
\end{gathered}
$$

e $D_{n}=A_{n} \cap B_{n} \cap C_{n}$. Então $\tilde{\xi}^{2, n}$ terá as seguintes propriedades

(a) Dado $D_{n}, \tilde{\xi}^{1, n}=\tilde{\xi}^{2, n} \mathrm{e}$

(b) $d_{\mathcal{H}_{-1}^{-\alpha}}\left(\tilde{\xi}^{1, n}, \tilde{\xi}^{2, n}\right) \underset{n \rightarrow \infty}{\stackrel{q \cdot c}{\longrightarrow}} 0$.

Vamos construir agora o conjunto de trajetórias $\tilde{\xi}^{2, n}$. Dado $z_{k} \in \hat{P}_{k}^{n} \cap \mathcal{B}_{n}, 0 \leq k \leq k_{n}$ temos que

$$
z_{k}=(n-k)\left(\sin \left(\frac{\epsilon_{z_{k}}}{n-k}\right),-\cos \left(\frac{\epsilon_{z_{k}}}{n-k}\right)\right)
$$

onde $J_{k, n}\left(\epsilon_{z_{k}}\right)=z_{k}, \epsilon_{z_{k}} \in P_{k}$ e $\left|\frac{\epsilon_{z_{k}}}{n-k}\right| \leq n^{\delta / 2-1 / 2} / 2$.

Seja $b_{0}\left(z_{k}\right)=\frac{\epsilon\left(z_{k}\right)}{n-k}$, definimos indutivamente

$$
b_{j}\left(z_{k}\right)=\frac{\arg \min _{x \in P_{j+k}}\left|b_{j-1}\left(z_{k}\right)-\frac{x}{n-j-k}\right|}{n-j-k},
$$

para $j=1,2, \ldots, k_{n}-k+1$. Note que dado $b_{j-1}\left(z_{k}\right)$, temos que $b_{j}\left(z_{k}\right)-b_{j-1}\left(z_{k}\right) \stackrel{\mathcal{D}}{=} \frac{\arg \min _{x \in P_{j+k}}|x|}{n-j-k}$.

Seja $\varsigma_{j}\left(z_{k}\right)=b_{j}\left(z_{k}\right)-b_{j-1}\left(z_{k}\right)$ para $j=1,2, \ldots, k_{n}-k+1$.

Seja $\tilde{\xi}_{z_{k}}^{2, n}$ a trajetória aleatória determinada pela interpolação linear dos pontos, 


$$
\left\{(n-k)\left(\frac{\sin \left(b_{0}\left(z_{k}\right)\right)}{\sqrt{n}},-\frac{1}{n}\right)\right\},\left\{(n-k-j)\left(\frac{\sin \left(b_{j}\left(z_{k}\right)\right)}{\sqrt{n}},-\frac{1}{n}\right)\right\}_{1 \leq j \leq k_{n}-k+1}
$$

restrita ao conjunto $\mathbb{R} \times[-1,-\alpha]$.

Seja

$$
\tilde{\xi}^{2, n}:=\left\{\tilde{\xi}_{z_{k}}^{2, n}: z_{k} \in \hat{P}_{k}^{n} \cap \mathcal{B}_{n}\right\}_{0 \leq k \leq k_{n}}
$$

Observação 4.2.1 $\tilde{\xi}^{1, n} \mathbb{I}_{\left\{D_{n}\right\}}=\tilde{\xi}^{2, n} \mathbb{I}_{\left\{D_{n}\right\}}$.

Lema 4.2.1 $d_{\mathcal{H}_{-1,-\alpha}}\left(\tilde{\xi}^{1, n}, \tilde{\xi}^{2, n}\right) \underset{n \rightarrow \infty}{\stackrel{q . c}{\rightarrow}} 0$.

Prova: Das propriedades 4,5 e 6 obtemos que $\mathbb{P}\left[D_{n}^{c}\right] \underset{n \rightarrow \infty}{\longrightarrow} 0$. O lema segui da observação 4.2.1.

\subsection{Terceira aproximação}

Precisamos de uma última aproximação ao conjunto de trajetórias $\tilde{\xi}^{n}$ que chamaremos $\tilde{\xi}^{3, n}$ tal que a distância em $\mathcal{H}_{-1,-\alpha}$ entre $\tilde{\xi}^{2, n}$ e $\tilde{\xi}^{3, n}$ vai para zero em probabilidade e novamente isto implica que o limite fraco terá que ser o mesmo.

Dado $z_{k} \in \hat{P}_{k}^{n} \cap \mathcal{B}_{n}$, seja $\tilde{\xi}_{z_{k}}^{3, n}$ a trajetória aleatória obtida por interpolação linear dos pontos,

$$
\left\{(n-k)\left(\frac{b_{0}\left(z_{k}\right)}{\sqrt{n}},-\frac{1}{n}\right)\right\},\left\{(n-k-j)\left(\frac{b_{j}\left(z_{k}\right)}{\sqrt{n}},-\frac{1}{n}\right)\right\}_{1 \leq j \leq k_{n}-k+1}
$$

restrita ao conjunto $\mathbb{R} \times[-1,-\alpha]$. Seja

$$
\tilde{\xi}^{3, n}:=\left\{\tilde{\xi}_{z_{k}}^{3, n}: z_{k} \in \hat{P}_{k}^{n} \cap \mathcal{B}_{n}\right\}_{0 \leq k \leq k_{n}}
$$

Seja $\rho_{k}(x, y):=\sup _{\{-(n-k) / n \leq t \leq-\alpha\}}|x(t)-y(t)|, \forall x, y \in \mathcal{C}[-(n-k) / n,-\alpha]$ para $k=0,1, \ldots, k_{n}+1$ 
Lema 4.3.1 $d_{\mathcal{H}_{-1,-\alpha}}\left(\tilde{\xi}^{2, n}, \tilde{\xi}^{3, n}\right) \underset{n \rightarrow \infty}{\stackrel{p}{\longrightarrow}} 0$.

Prova: Como cada trajetória de $\tilde{\xi}^{3, n}$ é identificada com exatamente uma trajetória de $\tilde{\xi}^{2, n}$, mostrar que

$$
d_{\mathcal{H}_{-1,-\alpha}}\left(\tilde{\xi}^{2, n}, \tilde{\xi}^{3, n}\right) \underset{n \rightarrow \infty}{\stackrel{p}{\longrightarrow}} 0
$$

é equivalente a mostrar o seguinte:

$$
\forall \epsilon>0, \mathbb{P}\left[\text { existe } z_{k} \in \hat{P}_{k}^{n} \cap \mathcal{B}_{n} \quad \text { tal que } \quad \rho_{k}\left(\tilde{\xi}_{z_{k}}^{2, n}, \tilde{\xi}_{z_{k}}^{3, n}\right)>\epsilon \text { para algum } k, 0 \leq k \leq k_{n}\right] \underset{n \rightarrow \infty}{\longrightarrow} 0
$$

Seja $\epsilon>0$ fixo, logo

$$
\begin{array}{r}
\mathbb{P}\left[\text { existe } z_{k} \in \hat{P}_{k}^{n} \cap \mathcal{B}_{n} \text { tal que } \rho_{k}\left(\tilde{\xi}_{z_{k}}^{2, n}, \tilde{\xi}_{z_{k}}^{3, n}\right)>\epsilon \text { para algum } k, 0 \leq k \leq k_{n}\right] \\
\leq \sum_{k=0}^{k_{n}} \mathbb{P}\left[\text { existe } z_{k} \in \hat{P}_{k}^{n} \cap \mathcal{B}_{n} \text { tal que } \rho_{k}\left(\tilde{\xi}_{z_{k}}^{2, n}, \tilde{\xi}_{z_{k}}^{3, n}\right)>\epsilon\right]
\end{array}
$$

Agora, se $z_{k} \in \hat{P}_{k}^{n} \cap \mathcal{B}_{n}$ é tal que $\rho_{k}\left(\tilde{\xi}_{z_{k}}^{2, n}, \tilde{\xi}_{z_{k}}^{3, n}\right)>\epsilon$, então necessariamente a distância entre algum dos pontos identificados tem que ser maior que $\epsilon / 2$ pois a interpolação é de tipo linear. Isto é, o evento

$$
\left\{\text { existe } z_{k} \in \hat{P}_{k}^{n} \cap \mathcal{B}_{n} \text { tal que } \rho_{k}\left(\tilde{\xi}_{z_{k}}^{2, n}, \tilde{\xi}_{z_{k}}^{3, n}\right)>\epsilon\right\}
$$

esta contido no evento

$$
\left\{\text { existe } z_{k} \in \hat{P}_{k}^{n} \cap \mathcal{B}_{n} \text { tal que } \sup _{0 \leq j \leq k_{n}-k+1}\left|\frac{n-k-j}{\sqrt{n}} b_{j}\left(z_{k}\right)-\frac{n-k-j}{\sqrt{n}} \sin \left(b_{j}\left(z_{k}\right)\right)\right|>\epsilon / 2\right\} .
$$

Assim, de (4.8),

$\mathbb{P}\left[\right.$ existe $z_{k} \in \hat{P}_{k}^{n} \cap \mathcal{B}_{n}$ tal que $\rho_{k}\left(\tilde{\xi}_{z_{k}}^{2, n}, \tilde{\xi}_{z_{k}}^{3, n}\right)>\epsilon$ para algum $\left.k, 0 \leq k \leq k_{n}\right] \leq$

$$
\sum_{k=0}^{k_{n}} \mathbb{P}\left[\text { existe } z_{k} \in \hat{P}_{k}^{n} \cap \mathcal{B}_{n} \text { tal que } \sup _{0 \leq j \leq k_{n}-k+1}\left|\frac{n-k-j}{\sqrt{n}} b_{j}\left(z_{k}\right)-\frac{n-k-j}{\sqrt{n}} \sin \left(b_{j}\left(z_{k}\right)\right)\right|>\epsilon / 2\right] \leq
$$




$$
\begin{aligned}
& \sum_{k=0}^{k_{n}} \mathbb{P}\left[\text { existe } z_{k} \in \hat{P}_{k}^{n} \cap \mathcal{B}_{n} \text { tal que } \sup _{0 \leq j \leq k_{n}-k+1} \frac{n-k-j}{\sqrt{n}}\left|b_{j}(z)-\sin \left(b_{j}(z)\right)\right|>\epsilon / 2\right] \leq \\
& \sum_{k=0}^{k_{n}} \mathbb{P}\left[\text { existe } z_{k} \in \hat{P}_{k}^{n} \cap \mathcal{B}_{n} \text { tal que } \sup _{0 \leq j \leq k_{n}-k+1} \frac{n-k-j}{n} \sqrt{n}\left|b_{j}(z)-\sin \left(b_{j}(z)\right)\right|>\epsilon / 2\right] \leq \\
& \sum_{k=0}^{k_{n}} \mathbb{P}\left[\text { existe } z_{k} \in \hat{P}_{k}^{n} \cap \mathcal{B}_{n} \text { tal que } \sup _{0 \leq j \leq k_{n}-k+1} \sqrt{n}\left|b_{j}(z)-\sin \left(b_{j}(z)\right)\right|>\epsilon / 2\right] \leq \\
& \sum_{k=0}^{k_{n}} \mathbb{P}\left[\text { existe } z_{k} \in \hat{P}_{k}^{n} \cap \mathcal{B}_{n} \text { tal que } \sup _{0 \leq j \leq k_{n}-k+1}\left|b_{j}(z)-\sin \left(b_{j}(z)\right)\right|>n^{-1 / 2} \epsilon / 2\right]
\end{aligned}
$$

Usando que $|\sin (x)-x| \leq C|x|^{3} \operatorname{com} C>0$, obtemos

$$
\begin{gathered}
\sum_{k=0}^{k_{n}} \mathbb{P}\left[\text { existe } z_{k} \in \hat{P}_{k}^{n} \cap \mathcal{B}_{n} \text { tal que } \sup _{0 \leq j \leq k_{n}-k+1}\left|b_{j}(z)-\sin \left(b_{j}(z)\right)\right|>n^{-1 / 2} \epsilon / 2\right] \leq \\
\sum_{k=0}^{k_{n}} \mathbb{P}\left[\text { existe } z_{k} \in \hat{P}_{k}^{n} \cap \mathcal{B}_{n} \text { tal que } \sup _{0 \leq j \leq k_{n}-k+1}\left|b_{j}(z)\right|^{3}>c_{0} n^{-1 / 2} \epsilon / 2\right] \leq \\
\sum_{k=0}^{k_{n}} \mathbb{P}\left[\text { existe } z_{k} \in \hat{P}_{k}^{n} \cap \mathcal{B}_{n} \text { tal que } \sup _{0 \leq j \leq k_{n}-k+1}\left|b_{j}(z)\right|>\left(c_{0} \epsilon / 2\right)^{1 / 3} n^{-1 / 6}\right],
\end{gathered}
$$

para alguma constante $c_{0}>0$, lembrando que

$$
b_{j}\left(z_{k}\right)=b_{0}\left(z_{k}\right)+\sum_{i=1}^{j} \varsigma_{i}\left(z_{k}\right), \quad j=1, \ldots, k_{n}-k+1
$$

e que $\left|b_{0}\left(z_{k}\right)\right| \leq n^{\delta / 2-1 / 2} / 2$ e $\delta \in(1 / 4,1 / 3)$, obtemos que,

$\mathbb{P}\left[\right.$ existe $z_{k} \in \hat{P}_{k}^{n} \cap \mathcal{B}_{n}$ tal que $\rho_{k}\left(\tilde{\xi}_{z_{k}}^{2, n}, \tilde{\xi}_{z_{k}}^{3, n}\right)>\epsilon$ para algum $\left.k, 0 \leq k \leq k_{n}\right] \leq$

$$
\sum_{k=0}^{k_{n}} \mathbb{P}\left[\text { existe } z_{k} \in \hat{P}_{k}^{n} \cap \mathcal{B}_{n} \text { tal que } \sup _{0 \leq j \leq k_{n}-k+1}\left|\sum_{i=1}^{j} \varsigma_{i}\left(z_{k}\right)\right|>\left(c_{0} \epsilon / 2\right)^{1 / 3} n^{-1 / 6} / 2\right]
$$

Sejam $C_{n}^{k}:=\left\{\right.$ existe $z_{k} \in \hat{P}_{k}^{n} \cap \mathcal{B}_{n}$ tal que $\left.\sup _{0 \leq j \leq k_{n}-k+1}\left|\sum_{i=1}^{j} \varsigma_{i}\left(z_{k}\right)\right|>c_{1} \epsilon^{1 / 3} n^{-1 / 6}\right\}$ para $k=0, \ldots, k_{n}$. Logo, 
$\mathbb{P}\left[\right.$ existe $z_{k} \in \hat{P}_{k}^{n} \cap \mathcal{B}_{n}$ tal que $\rho_{k}\left(\tilde{\xi}_{z_{k}}^{2, n}, \tilde{\xi}_{z_{k}}^{3, n}\right)>\epsilon$ para algum $\left.k, 0 \leq k \leq k_{n}\right] \leq \sum_{k=0}^{k_{n}} \mathbb{P}\left[C_{n}^{k}\right]$

Seja $W_{n}^{0}:=\left\{0<\left|\hat{P}_{0}^{n} \cap \mathcal{B}_{n}\right|<n\right\}$, então

$$
\begin{aligned}
\mathbb{P}\left[C_{n}^{0}\right] & =\mathbb{P}\left[C_{n}^{0} \mid W_{n}^{0}\right] \mathbb{P}\left[W_{n}^{0}\right]+\mathbb{P}\left[C_{n}^{0} \mid\left(W_{n}^{0}\right)^{c}\right] \mathbb{P}\left[\left(W_{n}^{0}\right)^{c}\right] \\
& \leq \mathbb{P}\left[C_{n}^{0} \mid W_{n}^{0}\right]+\mathbb{P}\left[\left(W_{n}^{0}\right)^{c}\right]
\end{aligned}
$$

Agora,

$$
\begin{aligned}
\mathbb{P}\left[\left(W_{n}^{0}\right)^{c}\right] & =\mathbb{P}\left[\left|\hat{P}_{k}^{n} \cap \mathcal{B}_{n}\right|=0\right]+\mathbb{P}\left[\left|\hat{P}_{0}^{n} \cap \mathcal{B}_{n}\right| \geq n\right] \\
& =e^{-n^{\delta / 2+1 / 2}}+\mathbb{P}\left[\left|\hat{P}_{0}^{n} \cap \mathcal{B}_{n}\right| \geq n\right]
\end{aligned}
$$

De (3.16) e (4.11) segue que existe uma constante $c_{2}>0$ tal que

$$
\mathbb{P}\left[\left(W_{n}^{0}\right)^{c}\right] \leq c_{2} e^{-n^{\delta / 2+1 / 2}}
$$

Além disso,

$$
\mathbb{P}\left[C_{n}^{0} \mid W_{n}^{0}\right]=\mathbb{P}\left[\text { existe } z_{0} \in \hat{P}_{0}^{n} \cap \mathcal{B}_{n} \text { tal que } \sup _{0 \leq j \leq k_{n}+1}\left|\sum_{i=1}^{j} \varsigma_{j}\left(z_{0}\right)\right|>c_{1} \epsilon^{1 / 3} n^{-1 / 6} \mid W_{n}^{0}\right]
$$

Lembrando que $\varsigma_{j}(z) \stackrel{\mathcal{D}}{=} \frac{\arg \min _{x \in P_{j}}|x|}{n-j}$ para $j=1,2, \ldots, k_{n}+1$, obtemos que

$$
\mathbb{P}\left[C_{n}^{0} \mid W_{n}^{0}\right] \leq n \mathbb{P}\left[\sup _{0 \leq j \leq k_{n}+1}\left|\sum_{i=1}^{j} \frac{\varepsilon_{i}}{n-j}\right|>c_{1} \epsilon^{1 / 3} n^{-1 / 6}\right]
$$

onde $\left(\varepsilon_{j}\right)_{j=1}^{k_{n}+1}$ são i.i.d com $\varepsilon_{1} \stackrel{\mathcal{D}}{=} \arg \min _{x \in P_{0}}|x|$, também como $\delta \in(1 / 4,1 / 3)$ temos que para $n$ suficientemente grande $c_{1} \epsilon^{1 / 3} n^{-1 / 6}>n^{\delta-1 / 2}$, então para $n$ suficientemente grande temos que

$$
\mathbb{P}\left[C_{n}^{0} \mid W_{n}^{0}\right] \leq n \mathbb{P}\left[\sup _{0 \leq j \leq k_{n}+1}\left|\sum_{i=1}^{j} \frac{\varepsilon_{i}}{n-j}\right|>n^{\delta-1 / 2}\right]
$$

de (3.22) e (4.14) segue que 


$$
\mathbb{P}\left[C_{n}^{0} \mid W_{n}^{0}\right] \leq n \times\left(n e^{-c_{3} n^{\delta}}+n e^{-c_{4} n^{2 \delta-1 / 2}}\right)=n^{2} e^{-c_{3} n^{\delta}}+n^{2} e^{-c_{4} n^{2 \delta-1 / 2}} .
$$

Assim, de (4.10), (4.12) e (4.15) obtemos

$$
\mathbb{P}\left[C_{n}^{0}\right] \leq n^{2} e^{-c_{3} n^{\delta}}+n^{2} e^{-n^{2 \delta-1 / 2}}+c_{2} e^{-n^{\delta / 2+1 / 2}} \leq c_{5} e^{-n^{2 \delta-1 / 2}} .
$$

Analogamente se pode mostrar que

$$
\mathbb{P}\left[C_{n}^{k}\right] \leq c_{5} e^{-n^{2 \delta-1 / 2}} \text {, para } k=0,1, \ldots, k_{n}+1
$$

Logo, de (4.11) segui que

$$
\begin{gathered}
\mathbb{P}\left[\text { existe } z_{k} \in \hat{P}_{k}^{n} \cap \mathcal{B}_{n} \text { tal que } \rho_{k}\left(\tilde{\xi}_{z_{k}}^{2, n}, \tilde{\xi}_{z_{k}}^{3, n}\right)>\epsilon \text { para algum } k, 0 \leq k \leq k_{n}\right] \leq \\
c_{5} \sum_{k=0}^{k_{n}} e^{-n^{2 \delta-1 / 2}} \leq c_{5} n e^{-n^{2 \delta-1 / 2}},
\end{gathered}
$$

o resultado segue do fato que $\delta \in(1 / 4,1 / 3)$.

\subsection{Convergência de uma única trajetória}

Vamos mostrar agora a convergência de uma única trajetória de $\tilde{\xi}^{3, n}$. Isto é importante pois mostrará que com as propriedades da Teia Browniana restrita, a transformação que precisamos é aquela mostrada no Teorema 3.3.1.

Lembremos que $\mathcal{B}_{n}:=\left\{z \in \mathbb{R} \times[-n,-n \alpha]:\left|\tilde{\theta}_{z}\right| \leq n^{\delta / 2-1 / 2} / 2\right\}, \operatorname{assim} E^{n}\left[\mathcal{B}_{n}\right]=\left\{\left(\frac{x}{\sqrt{n}}, \frac{y}{n}\right):(x, y) \in \mathcal{B}_{n}\right\}$, é fácil mostrar a seguinte observação.

Observação 4.4.1 $\cup_{n=1}^{\infty} E^{n}\left[\mathcal{B}_{n}\right]=\mathbb{R} \times[-1,-\alpha]$

Lema 4.4.1 Dado $\left(x_{0}, t_{0}\right) \in \mathbb{R} \times[-1,-\alpha]$ fixo, temos que

$$
\mathbb{P}\left[\inf _{z_{\left\lfloor n\left(1+t_{0}\right)\right\rfloor} \in \hat{P}_{\left\lfloor n\left(1+t_{0}\right)\right\rfloor}^{n} \cap \mathcal{B}_{n}}\left\|\tilde{\xi}_{z_{\left\lfloor n\left(1+t_{0}\right)\right\rfloor}^{3, n}}^{3,}(0)-\left(x_{0}, t_{0}\right)\right\|>2 n^{\delta / 2-1 / 2} \quad i . v\right]=0 .
$$

Prova: Seja $\left(x_{0}, t_{0}\right) \in \mathbb{R} \times[-1,-\alpha]$. Para $n$ suficientemente grande obtemos: 


$$
\left(\sqrt{n} x_{0}-n^{\delta}, \sqrt{n} x_{0}+n^{\delta}\right) \subset\left(-n^{\delta / 2-1 / 2}\left[n-\left\lfloor n\left(1+t_{0}\right)\right\rfloor\right], n^{\delta / 2-1 / 2}\left[n-\left\lfloor n\left(1+t_{0}\right)\right\rfloor\right]\right)
$$

Seja $\tilde{x}^{n}=\left(x_{0},-\frac{n-\left\lfloor n\left(1+t_{0}\right)\right\rfloor}{n}\right), \operatorname{logo}$

$$
\left\|\tilde{x}^{n}-\left(x_{0}, t_{0}\right)\right\| \leq \frac{1}{n}
$$

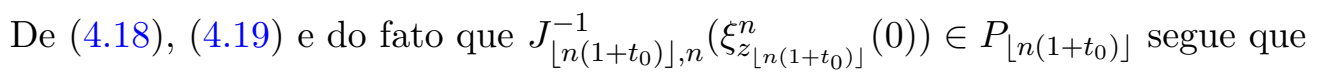

$$
\left\{\inf _{z_{\left\lfloor n\left(1+t_{0}\right)\right\rfloor} \in \hat{P}_{\left\lfloor n\left(1-t_{0}\right)\right\rfloor}^{n} \cap \mathcal{B}_{n}}\left\|\tilde{\xi}_{z_{\left\lfloor n\left(1+t_{0}\right)\right\rfloor}^{3, n}}(0)-\left(x_{0}, t_{0}\right)\right\|>2 n^{\delta / 2-1 / 2}\right\} \subset\left\{P_{\left\lfloor n\left(1+t_{0}\right)\right\rfloor} \cap\left(\sqrt{n} x_{0}-n^{\delta}, \sqrt{n} x_{0}+n^{\delta}\right)=\emptyset\right\}
$$

assim,

$$
\mathbb{P}\left[\inf _{z \in \hat{P}_{\left\lfloor n\left(1+t_{0}\right)\right\rfloor} \cap \mathcal{B}_{n}}\left\|\tilde{\xi}_{z_{\left\lfloor n\left(1+t_{0}\right)\right\rfloor}^{3, n}}^{3,}(0)-\left(x_{0}, t_{0}\right)\right\|>2 n^{\delta / 2-1 / 2}\right] \leq e^{-2 n^{\delta}}
$$

O lema segue do Lema de Borel-Cantelli.

Vamos mostrar agora a convergência fraca para uma trajetória saindo de um ponto em $\mathbb{R} \times[-1,-\alpha]$

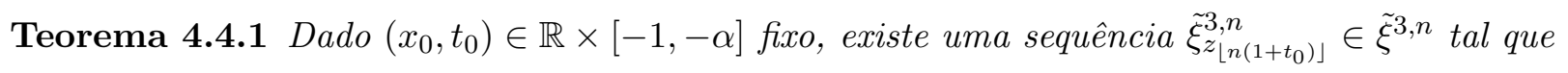

$$
\tilde{\xi}_{z\left\lfloor n\left(1+t_{0}\right)\right\rfloor}^{3, n} \underset{n \rightarrow \infty}{\stackrel{\mathcal{D}}{\longrightarrow}} t\left(\frac{x_{0}}{t_{0}}+c B[g(t)]\right), \quad \text { em } \quad \mathcal{C}\left[t_{0},-\alpha\right]
$$

onde para $t$ fixo, $B[g(t)] \stackrel{\mathcal{D}}{=} N(0, g(t)),\{B[g(t)]\}_{t \in\left[t_{0},-\alpha\right]}$ é um processo com incrementos independentes, $c=\sqrt{2 \operatorname{Var}\left[\arg \min _{x \in P_{0}}|x|\right]}$ e $g(t)=\int_{t_{0}}^{t} \frac{1}{x^{2}} d x=\frac{1}{|t|}-\frac{1}{\left|t_{0}\right|}$.

Prova: Se $t_{0}=-\alpha$, a prova segue do lema anterior, isto é, teríamos convergência quase certa para um ponto.

Vamos fazer a prova para $\tilde{x}=(0,-1)$ para qualquer outro ponto a demonstração é análoga. Do lema anterior é suficiente com encontrar a convergência em distribuição da sequência de trajetórias aleatórias obtidas por interpolação linear dos pontos,

$$
\{(0,-1)\},\left\{\left(\frac{(n-j)}{n} \sum_{i=1}^{j} \sqrt{n} \varsigma_{i}\left(\tilde{x}^{n}\right),-\frac{n-j}{n}\right)\right\}_{j=1}^{k_{n}+1}
$$


restrita ao espaço $\mathbb{R} \times[-1,-\alpha]$, onde $\varsigma_{i}=\frac{\arg \min _{x \in P_{i}}|x|}{n-i}$. Pelo Teorema A.0.2 temos que a sequência converge fracamente ao processo

$$
\left(c(1-t) B\left[\frac{1}{1-t}-1\right], t-1\right), \quad \text { em } \quad \mathcal{C}[0,1-\alpha]
$$

que é igual em distribuição ao processo

$$
\left(\operatorname{ct} B\left[\frac{1}{|t|}-1\right], t\right), \quad-1 \leq t \leq-\alpha
$$

Observação 4.4.2 No teorema anterior, se mostra como deve ser a transformação que precisamos, toda vez que ela tem que mapear o objeto limite anterior, num movimento Browniano saindo de algum ponto.

\subsection{Transformação adequada.}

Agora trabalharemos com a Teia Browniana restrita (ver 2.2). Nosso objetivo é mostrar que existe um homeomorfismo $T$ de $\mathcal{H}_{-1,-\alpha}$ em $\mathcal{H}_{0,1 / \alpha-1}$ tal que $T\left[\tilde{\xi}^{3, n}\right] \underset{n \rightarrow \infty}{\stackrel{\mathcal{D}}{\longrightarrow}} \overline{\mathcal{W}}_{0,1 / \alpha-1}$ e portanto $\tilde{\xi}^{3, n} \underset{n \rightarrow \infty}{\stackrel{\mathcal{D}}{\longrightarrow}} T^{-1}\left[\overline{\mathcal{W}}_{0,1 / \alpha-1}\right]$.

Considere o homeomorfismo $T$ de $\mathcal{H}_{-1,-\alpha}$ em $\mathcal{H}_{0,1 / \alpha-1}$, como em (3.27).

Lembremos que $\tilde{\xi}_{z_{k}}^{3, n}$ é a trajetória aleatória obtida por interpolação linear dos pontos

$$
\left\{\left(\frac{n-k}{n} \sqrt{n} b_{0}\left(z_{k}\right),-\frac{n-k}{n}\right),\left(\frac{n-k-j}{n} \sqrt{n} b_{j}\left(z_{k}\right),-\frac{n-k-j}{n}\right)_{j=1}^{k_{n}-k+1}\right\}
$$

Portanto, $T\left(\tilde{\xi}_{z_{k}}^{3, n}\right)$ é a trajetória cujos pontos de interpolação devida a $T$ são

$$
\left\{\left(\sqrt{n} b_{0}\left(z_{k}\right), \frac{k}{n-k}\right),\left(\sqrt{n} b_{j}\left(z_{k}\right),-\frac{k+j}{n-(k+j)}\right)_{j=1}^{k_{n}-k+1}\right\}
$$

Do Teorema 4.4.1, dado $\left(x_{0}, t_{0}\right) \in \mathbb{R} \times[-1,-\alpha]$ existe uma sequência $\tilde{\xi}_{z\left\lfloor n\left(1+t_{0}\right)\right\rfloor}^{3, n} \in \tilde{\xi}^{3, n}$ tal que

$$
\tilde{\xi}_{z\left\lfloor n\left(1+t_{0}\right)\right\rfloor}^{3, n \rightarrow \infty} \underset{n \rightarrow}{\stackrel{\mathcal{D}}{\longrightarrow}} t\left\{\frac{x_{0}}{t_{0}}+c B\left[\frac{1}{|t|}-\frac{1}{\left|t_{0}\right|}\right]\right\}, \quad \text { em } \quad \mathcal{C}\left[t_{0},-\alpha\right] .
$$


Logo, do Teorema do mapeamento continuo

$$
T\left[\tilde{\xi}_{\left.z_{\left\lfloor n\left(1+t_{0}\right)\right\rfloor}^{3, n}\right]}^{\underset{n \rightarrow \infty}{\mathcal{D}}}\left(\frac{x_{0}}{t_{0}}+c B\left[\frac{1}{|t|}-\frac{1}{\left|t_{0}\right|}\right], \frac{1}{|t|}-1\right), \quad \text { em } \quad \mathcal{C}\left[t_{0},-\alpha\right] .\right.
$$

Seja $t^{\prime}=\frac{1}{|t|}-1$, então

$$
T\left[\tilde{\xi}_{\left.z_{\left\lfloor n\left(1+t_{0}\right)\right\rfloor}^{3, n}\right]}^{\stackrel{\mathcal{D}}{\longrightarrow}}\left(\frac{x_{0}}{t_{0}}+c B\left[t^{\prime}-t_{0}^{\prime}\right], t^{\prime}\right), \quad \text { em } \quad \mathcal{C}\left[t_{0}^{\prime}, 1 / \alpha-1\right] .\right.
$$

$\operatorname{com} t_{0}^{\prime}=\frac{1}{\left|t_{0}\right|}-1$

Vamos a denotar a $T\left[\tilde{\xi}^{3, n}\right]$ como

$$
Y^{n}:=T\left[\tilde{\xi}^{3, n}\right]
$$

Assim, temos o seguinte lema,

Lema 4.5.1 Dado $\left(x_{0}^{\prime}, t_{0}^{\prime}\right) \in \mathbb{R} \times[0,1 / \alpha-1]$ existe uma sequência $Y_{\left(x_{0}^{\prime}, t_{0}^{\prime}\right)}^{n} \in Y^{n}$ tal que

$$
Y_{\left(x_{0}^{\prime}, t_{0}^{\prime}\right)}^{\stackrel{\mathcal{D}}{\longrightarrow}} \underset{n \rightarrow \infty}{\longrightarrow}\left(x_{0}^{\prime}+c B\left[t-t_{0}^{\prime}\right], t^{\prime}\right), \quad \text { em } \quad \mathcal{C}\left[t_{0}^{\prime}, 1 / \alpha-1\right]
$$

Observação 4.5.1 Seja $m_{k}=\frac{k}{n-k}$ e $E_{k}=\left[-n^{\delta / 2-1}(n-k), n^{\delta / 2-1}(n-k)\right] \times\left\{m_{k}\right\}, 0 \leq k \leq k_{n}+1$. Sejam $\left(R_{k}\right)_{k=1}^{k_{n}+1}$ processos de Poisson independentes em $\mathbb{R}, R_{k}$ com taxa $\frac{n-k}{\sqrt{n}}$. Dado $u \in E_{k}$, $k=1,2, \ldots, k_{n}$, denotamos por

$$
u^{1, k}=\arg \min _{v \in R_{k+1} \times\left\{m_{k+1}\right\}}\|u-v\|
$$

e indutivamente,

$$
u^{j, k}=\arg \min _{v \in R_{k+j} \times\left\{m_{k+j}\right\}}\left\|u^{j-1, k}-v\right\|
$$

onde $u^{0, k}=u$ e $j=1, \ldots, k_{n}-k+1$. Então, seja $\hat{Y}_{u}^{n, T}$ a trajetória aleatória obtida de interpolar os pontos 


$$
\left\{u, u^{1, k}, \ldots, u^{k_{n}-k+1}\right\}
$$

usando $T$, isto é, logo de interpolar linearmente os pontos $\left\{T^{-1}(u), T^{-1}\left(u^{1, k}\right), \ldots, T^{-1}\left(u^{k_{n}-k+1}\right)\right\}$ denotamos (como um abuso de notação) a trajetória restrita ao conjunto $\mathbb{R} \times[-1,-\alpha]$ como $\tilde{\xi}_{T^{-1}}^{3, n}(u)$ e $\hat{Y}_{u}^{n, T}=T\left[\tilde{\xi}_{T^{-1}(u)}^{3, n}\right]$. Seja

$$
\hat{Y}^{n, T}=:\left\{\hat{Y}_{u}^{n, T}: u \in \cup_{k=0}^{k_{n}} E_{k} \cap\left(R_{k} \times\left\{m_{k}\right\}\right)\right\}
$$

segue que $\hat{Y}^{n, T} \stackrel{\mathcal{D}}{=} Y^{n}$. 


\section{Capítulo 5}

\section{Prova do teorema principal}

Para mostrar o Teorema 3.3.1, precisamos provar que $\hat{Y}^{n, T}\left(\hat{Y}^{n, T} \stackrel{\mathcal{D}}{=} T\left[\tilde{\xi}^{3, n}\right]\right)$ converge em distribuição à Teia Browniana radial restrita, isto é, $\hat{Y}^{n, T}$ satisfaz as condições $(I),\left(B_{1}\right)$ e $\left(B_{2}\right)$ no Teorema 2.2.2. Para esta finalidade, usaremos dois lemas da secção seguinte

\subsection{Tempo de Coalescência}

Sejam $\left(Q_{k}\right)_{k=0}^{\infty}$ processos de Poisson em $\mathbb{R}$ independentes, $Q_{k}$ com taxa $\frac{n-k}{n}$ para $k=0,1, \ldots, k_{n}$ e para $Q_{k}$ com $k>k_{n}$ taxa $\frac{n-k_{n}}{n}$. Considere o subconjunto de pontos em $\mathbb{R}^{2}$ formado por $\cup_{k=0}^{\infty} Q_{k} \times$ $\{k\}$.

Dado um ponto $u=\left(u_{0}, k\right) \in \mathbb{R} \times\{k\}$, seja $\tilde{u}^{1, k}=\arg \min _{v \in Q_{k+1} \times\{k+1\}}\|u-v\|$ e indutivamente

$$
\tilde{u}^{j, k}=\arg \min _{v \in Q_{k+j+1} \times\{k+j+1\}}\left\|\tilde{u}^{j-1, k}-v\right\| \quad j=1,2, \ldots
$$

onde $\tilde{u}^{0, k}=u$. Seja $X^{u}$ a trajetória obtida de interpolar linearmente os pontos $\left\{u, \tilde{u}^{1, k}, \ldots, \tilde{u}^{j, k}, \ldots\right\}$.

Sejam $u, w \in \mathbb{R} \times\{0\} \operatorname{com} u=\left(u_{1}, 0\right), w=\left(w_{1}, 0\right)$ e $u_{1}<v_{1}$. Considere $X^{u}, X^{w}$ e para $t \geq 0$,

$$
Z_{t}=Z_{t}(u, w)=X_{t}^{v}-X_{t}^{w}
$$

Suponhamos que $w_{1}-u_{1}=1$. Definimos

$$
\tau:=\min \left\{t \geq 0: Z_{t}=0\right\}
$$


Proposição 5.1.1 Existe uma constante $C>0$, tal que $\mathbb{P}[\tau>t] \leq \frac{C}{\sqrt{t}}$.

Prova: Como $\left\{Z_{t}(u, v), t \geq 0\right\}$ é um Martingal não negativo em $\mathbb{L}^{2}$, pelo Teorema de representação de Skorohod existe um movimento Browniano com coeficiente de difusão 1 começando em 1 e tempos de parada $0=T_{0}, T_{1}, T_{2}, \ldots$ satisfazendo:

$$
Z_{t} \stackrel{\mathcal{D}}{=} B\left[T_{t}\right]
$$

onde $0=T_{0}, T_{1}, T_{2}, \ldots$ são tais que

$$
T_{t}=\inf \left\{s \geq T_{t-1}: B[s]-B\left[T_{t-1}\right] \notin\left(U_{t}\left(B\left(T_{t-1}\right)\right), V_{t}\left(B\left[T_{t-1}\right]\right)\right)\right\},
$$

onde $\left\{\left(U_{t}(r), V_{t}(r)\right), t \geq 1, r \in[0, \infty)\right\}$ é uma familia de vetores aleatórios independentes e no presente caso, para todo $r \geq 0, t, t^{\prime} \geq k_{n},\left(U_{t}(r), V_{t}(r)\right) \stackrel{\mathcal{D}}{=}\left(U_{t^{\prime}}(r), V_{t^{\prime}}(r)\right)$ e $\left(U_{t}(r), V_{t}(r)\right) \in[-r, 0) \times$ $(0, \infty)$ q.c.

Seja $\tau^{\prime}:=\inf \{t \geq 0: B[t]=0\}$. Temos que $\tau>t$ se e somente se $\tau^{\prime}>T_{t}$, logo

$$
\mathbb{P}[\tau>t]=\mathbb{P}\left[\tau^{\prime}>T_{t}\right]
$$

Dado $\varsigma>0$ por determinar, seguindo as ideias em [2], temos que

$$
\begin{aligned}
\mathbb{P}\left[\tau^{\prime}>T_{t}\right] & =\mathbb{P}\left[\left\{\tau^{\prime}>T_{t}\right\} \cap\left\{T_{t}>\varsigma t\right\}\right]+\mathbb{P}\left[\left\{\tau^{\prime}>T_{t}\right\} \cap\left\{T_{t} \leq \varsigma t\right\}\right] \\
& \leq \mathbb{P}\left[\tau^{\prime}>\varsigma t\right]+\mathbb{P}\left[\left\{\tau^{\prime}>T_{t}\right\} \cap\left\{T_{t} \leq \varsigma t\right\}\right] \\
& \leq \frac{c_{0}}{\sqrt{t}}+\mathbb{P}\left[\left\{\tau^{\prime}>T_{t}\right\} \cap\left\{T_{t} \leq \varsigma t\right\}\right]
\end{aligned}
$$

onde $c_{0}=c_{0}(\varsigma) \in(0, \infty)$. Agora, dado $\lambda>0$ por determinar

$$
\begin{aligned}
\mathbb{P}\left[\left\{\tau^{\prime}>T_{t}\right\} \cap\left\{T_{t} \leq \varsigma t\right\}\right] & \leq \mathbb{P}\left[\left\{\tau^{\prime}>T_{t}\right\} \cap\left\{e^{-\lambda T_{t}} \geq e^{-\lambda \varsigma t}\right\}\right] \\
& =\mathbb{P}\left[\left\{\Pi_{i=1}^{t} \mathbb{I}_{\left\{Z_{i}>0\right\}}>0\right\} \cap\left\{e^{-\lambda T_{t}} \geq e^{-\lambda \varsigma t}\right\}\right] \\
& =\mathbb{P}\left[\Pi_{i=1}^{t} \mathbb{I}_{\left\{Z_{i}>0\right\}} e^{-\lambda T_{t}} \geq e^{-\lambda \varsigma t}\right] \\
& \leq e^{\lambda \varsigma t} \mathbb{E}\left[\Pi_{i=1}^{t} \mathbb{I}_{\left\{Z_{i}>0\right\}} e^{-\lambda T_{t}}\right]
\end{aligned}
$$

Note que, $T_{t}=\sum_{i=1}^{t}\left(T_{i}-T_{i-1}\right)=\sum_{i=1}^{t} S_{i}\left(Z_{i-1}\right)$ onde $\left(S_{i}(r), i \in \mathbb{N}, r>0\right)$ são variáveis aleatórias independentes, entanto para $r>0,\left(S_{i}(r), i \in \mathbb{N}\right)$ não são identicamente distribuídas. Então, 


$$
\begin{aligned}
\mathbb{E}\left[\Pi_{i=1}^{t} \mathbb{I}_{\left\{Z_{i}>0\right\}} e^{-\lambda T_{t}}\right] & =\mathbb{E}\left[\Pi_{i=1}^{t} \mathbb{I}_{\left\{Z_{i}>0\right\}} \exp \left\{-\lambda \sum_{i=1}^{t} S_{i}\left(Z_{i-1}\right)\right\}\right] \\
& =\mathbb{E}\left[\mathbb{E}\left(\Pi_{i=1}^{t-1} \mathbb{I}_{\left\{Z_{i}>0\right\}} \exp \left\{-\lambda \sum_{i=1}^{t-1} S_{i}\left(Z_{i-1}\right)\right\} \mathbb{I}_{\left\{Z_{t}>0\right\}} \exp \left\{-\lambda S_{t}\left(Z_{t-1}\right)\right\} \mid \mathcal{F}_{t-1}\right)\right] \\
& \leq \sup _{r>0} \mathbb{E}\left[\mathbb{I}_{\left\{Z_{t}>0\right\}} e^{-\lambda S_{t}\left(Z_{t-1}\right)} \mid Z_{t-1}=r\right] \mathbb{E}\left[\Pi_{i=1}^{t-1} \mathbb{I}_{\left\{Z_{i}>0\right\}} \exp \left\{-\lambda \sum_{i=1}^{t-1} S_{i}\left(Z_{i-1}\right)\right\}\right] \\
& \leq \Pi_{i=1}^{t} \sup _{r>0} \mathbb{E}\left[\mathbb{I}_{\left\{Z_{i}>0\right\}} e^{-\lambda S_{i}\left(Z_{i-1}\right)} \mid Z_{i-1}=r\right]
\end{aligned}
$$

onde $\left\{\mathcal{F}_{t}\right\}$ é a sigma álgebra gerada por $Z_{0}, Z_{1}, \ldots, Z_{t}$.

Note que $\mathbb{E}\left[\mathbb{I}_{\left\{Z_{t}>0\right\}} e^{-\lambda S_{t-1}\left(Z_{t-1}\right)} \mid Z_{t-1}=r\right] \leq \mathbb{P}\left[Z_{t}>0 \mid Z_{t-1}=r\right]$. Isto é, $\mathbb{E}\left[\mathbb{I}_{\left\{Z_{t}>0\right\}} e^{-\lambda S_{t-1}\left(Z_{t-1}\right)} \mid Z_{t-1}=r\right]$ é limitado superiormente pela probabilidade do evento de não coalescência no nível $t$ dado que a distancia entre os processos no nível $t-1$ foi de $r$. Assim,

$$
\sup _{r \leq 10} \mathbb{E}\left[\mathbb{I}_{\left\{Z_{1}>0\right\}} e^{-\lambda S_{1}\left(Z_{t-1}\right)} \mid Z_{t-1}=r\right] \leq \sup _{r \leq 10} \mathbb{P}\left[Z_{t}>0 \mid Z_{t-1}=r\right]=\mathbb{P}\left[Z_{t}>0 \mid Z_{t-1}=10\right] .
$$

Devido a invariância por translação dos processos de Poisson temos que $0<r_{1}<r_{2}$ implica que $\mathbb{P}\left[Z_{t}>0 \mid Z_{t-1}=r_{1}\right] \leq \mathbb{P}\left[Z_{t}>0 \mid Z_{t-1}=r_{2}\right]$, isto justifica a igualdade no lado direito de (5.9).

Definição 5.1.1 Dado $x \in \mathbb{R}$ fixo, seja $p(x)=\arg \min _{y \in Q_{t}}|y-x|$.

Seja $B:=\{p(0) \neq p(10)\}$, devido a invariância por translação dos processos de Poisson temos que

$$
\mathbb{P}[B]=\mathbb{P}\left[Z_{t}>0 \mid Z_{t-1}=10\right]
$$

Agora, considere o evento

$$
C=\left\{\left|[-10,0] \cap Q_{t}\right|=0\right\} \cap\left\{\left|(0,10) \cap Q_{t}\right|=1\right\} \cap\left\{\left|[10,20] \cap Q_{t}\right|=0\right\}
$$

Claramente $C \subset B^{c}$. Seja $\kappa_{t}$ a taxa do processo $Q_{t}$.

$$
\mathbb{P}[C]=e^{-\kappa_{t} 20} e^{-\kappa_{t} 10} \kappa_{t} 10 \geq e^{-30} \alpha 10=c_{0}>0 .
$$

pois $\kappa_{t} \in[\alpha, 1]$. Note que $c_{0}$ não depende de $t$. Lembrando que $\mathbb{P}[B]=\mathbb{P}\left[Z_{t}>10 \mid Z_{t-1}=10\right]$, obtemos que $\mathbb{P}\left[Z_{t}>0 \mid Z_{t-1}=10\right] \leq 1-c_{0}=c_{1}<1$. De $(5.9)$, 


$$
\sup _{r \leq 10} \mathbb{E}\left[\mathbb{I}_{\left\{Z_{t}>0\right\}} e^{-\lambda S_{t}\left(Z_{t-1}\right)} \mid Z_{t-1}=r\right] \leq c_{1}<1
$$

Agora, queremos mostrar que existe uma constante $c_{2}<1$ que não depende de $t$ tal que

$$
\sup _{r \geq 10} \mathbb{E}\left[e^{-\lambda S_{t}(r)}\right] \leq c_{2}
$$

De aqui para o frente consideremos unicamente o caso $r \geq 10$.

A estratégia para mostrar (5.11) é escolher adequadamente um conjunto $R_{\epsilon_{0}}$ tal que

$$
\mathbb{E}\left[e^{-\lambda S_{t}(r)}\right] \leq \mathbb{P}\left[\left(U_{t}(r), V_{t}(r)\right) \in R_{\epsilon_{0}}\right]\left(1-a\left(\epsilon_{0}\right)\right)+a\left(\epsilon_{0}\right)
$$

onde $a\left(\epsilon_{0}\right)$ e $\mathbb{P}\left[\left(U_{t}(r), V_{t}(r)\right) \in R_{\epsilon_{0}}\right] \in(0,1)$ não dependem de $t, r$.

Seja $\epsilon>0$ dado, considere os conjuntos $A_{\epsilon}:=(-\epsilon, 0) \times(0, \infty)$ e $B_{\epsilon}:=[-r, 0) \times(0, \epsilon)$. Seja $F^{t}(x)=\mathbb{P}\left[Z_{t}-r \leq x \mid Z_{t-1}=r\right]$. Em [6] pag 403, temos que:

$$
\begin{gathered}
\mathbb{P}\left[\left(U_{t}(r), V_{t}(r)\right) \in A_{\epsilon} \cup B_{\epsilon}\right]=\frac{1}{c} \iint_{(u, v) \in A_{\epsilon} \cup B_{\epsilon}}(v-u) d F^{t}(u) d F^{t}(v) \\
\leq \frac{1}{c} \iint_{(u, v) \in(-\epsilon, 0) \times(0, \infty)}(v-u) d F^{t}(u) d F^{t}(v)+\frac{1}{c} \iint_{(u, v) \in[-r, 0) \times(0, \epsilon)}(v-u) d F^{t}(u) d F^{t}(v)
\end{gathered}
$$

onde $c=c(r)=\int_{-r}^{0}(-u) d F^{t}(u)=\int_{0}^{\infty} v d F^{t}(v)$, note que $c<\infty$ pois os incrementos são devidos ao ponto do processo de Poisson mais perto assim a distribuição tem cauda exponencial.

$$
\begin{gathered}
\frac{1}{c} \iint_{(u, v) \in(-\epsilon, 0) \times(0, \infty)}(v-u) d F^{t}(u) d F^{t}(v) \\
=\frac{1}{c} \iint_{(u, v) \in(-\epsilon, 0) \times(0, \infty)} v d F^{t}(u) d F^{t}(v)+\frac{1}{c} \iint_{(u, v) \in(-\epsilon, 0) \times(0, \infty)}(-u) d F^{t}(u) d F^{t}(v) \\
\leq \int_{u \in(-\epsilon, 0)} d F^{t}(u)+\frac{1}{c} \int_{u \in(-\epsilon, 0)}(-u) d F^{t}(u)=\mathbb{P}\left[-\epsilon \leq Z_{t}-r \leq 0 \mid Z_{t-1}=r\right]+\frac{1}{c} \int_{u \in(-\epsilon, 0)}(-u) d F^{t}(u)
\end{gathered}
$$

Portanto,

$$
\frac{1}{c} \iint_{(u, v) \in(-\epsilon, 0) \times(0, \infty)}(v-u) d F^{t}(u) d F^{t}(v) \leq \mathbb{P}\left[-\epsilon \leq Z_{t}-r \leq 0 \mid Z_{t-1}=r\right]+\frac{\epsilon}{c}
$$


Analogamente,

$$
\frac{1}{c} \iint_{(u, v) \in[-r, 0) \times(0, \epsilon)}(v-u) d F^{t}(u) d F^{t}(v) \leq \mathbb{P}\left[0 \leq Z_{t}-r \leq \epsilon \mid Z_{t-1}=r\right]+\frac{\epsilon}{c} .
$$

Assim, de (5.12), (5.13) e (5.14) temos que

$$
\mathbb{P}\left[\left(U_{t}(r), V_{t}(r)\right) \in A_{\epsilon} \cup B_{\epsilon}\right] \leq \mathbb{P}\left[-\epsilon \leq Z_{t}-r \leq \epsilon \mid Z_{t-1}=r\right]+\frac{2 \epsilon}{c} .
$$

De novo, pela invariância por translação dos processos de Poisson

$$
\mathbb{P}\left[-\epsilon \leq Z_{t}-r \leq \epsilon \mid Z_{t-1}=r\right]=\mathbb{P}[-\epsilon \leq p(r)-p(0)-r \leq \epsilon]
$$

Lembremos que $r \geq 10$. Considere os eventos, $C_{1}:=\left\{[-10 / 4,10 / 4] \cap Q_{t} \neq \emptyset\right\}$ e $C_{2}:=\{[-10 / 4+$ $\left.r, r+10 / 4] \cap Q_{t} \neq \emptyset\right\}$ portanto,

$$
\begin{aligned}
\mathbb{P}[-\epsilon \leq p(r)-p(0)-r \leq \epsilon] & \leq \mathbb{P}\left[-\epsilon \leq p(r)-p(0)-r \leq \epsilon \mid C_{1} \cap C_{2}\right] \mathbb{P}\left[C_{1} \cap C_{2}\right]+\mathbb{P}\left[C_{1}{ }^{c} \cup C_{2}{ }^{c}\right] \\
& =\mathbb{P}\left[-\epsilon \leq p(r)-p(0)-r \leq \epsilon \mid C_{1} \cap C_{2}\right]\left(1-e^{-5 \kappa_{t}}\right)^{2}+\left[1-\left(1-e^{-5 \kappa_{t}}\right)^{2}\right]
\end{aligned}
$$

Dado $C_{1} \cap C_{2}, p(r)$ e $p(0)$ ficam independentes, assim

$$
\mathbb{P}\left[-\epsilon \leq p(r)-p(0)-r \leq \epsilon \mid C_{1} \cap C_{2}\right] \leq \mathbb{P}\left[\left|[-\epsilon / 2, \epsilon / 2] \cap Q_{t}\right| \geq 1\right]
$$

Portanto

$$
\mathbb{P}\left[-\epsilon \leq p(r)-p(0)-r \leq \epsilon \mid C_{1} \cap C_{2}\right] \leq 1-e^{-\kappa_{t} \epsilon} .
$$

Assim de (5.15),(5.16) e (5.17) segue que

$$
\sup _{r \geq 10} \mathbb{P}\left[\left(U_{t}(r), V_{t}(r)\right) \in A_{\epsilon} \cup B_{\epsilon}\right] \leq\left(1-e^{-k_{t} \epsilon}\right)\left(1-e^{-5 \kappa_{t}}\right)^{2}+\left[1-\left(1-e^{-5 \kappa_{t}}\right)^{2}\right]+\frac{2 \epsilon}{c} .
$$

Dado que $\kappa_{t} \in[\alpha, 1]$, obtemos

$$
\sup _{r \geq 10} \mathbb{P}\left[\left(U_{t}(r), V_{t}(r)\right) \in A_{\epsilon} \cup B_{\epsilon}\right] \leq\left(1-e^{-\epsilon}\right)\left(1-e^{-5}\right)^{2}+\left[1-\left(1-e^{-5 \alpha}\right)^{2}\right]+\frac{2 \epsilon}{c} .
$$

Lembrando que $c=c(r)=\int_{-r}^{0}(-u) d F^{t}(u)=\int_{0}^{\infty} v d F^{t}(v)$, note que $c$ depende do nível $t$ e de $r$, mais para valores de $r$ superiores a 10 (de fato maior que qualquer constante positiva fixa), $c$ pode ser limitada inferiormente por uma constante que não depende de $t$ e tampouco depende de $r$. Vejamos, 


$$
\begin{aligned}
c=\int_{0}^{\infty} v d F^{t}(v) & \geq \int_{1}^{\infty} v d F^{t}(v) \\
& \geq \mathbb{P}\left[Z_{t}-r \geq 1 \mid Z_{t-1}=r\right]
\end{aligned}
$$

De novo, pela invariância por translação temos que

$$
\mathbb{P}\left[Z_{t}-r \geq 1 \mid Z_{t-1}=r\right]=\mathbb{P}[p(r)-p(0) \geq r+1]
$$

Lembrando que $r \geq 10$. Considere o evento, $D=\left\{\left|[1,-2] \cap Q_{t}\right|=1\right\} \cap\left\{\left|[-1,10] \cap Q_{t}\right|=0\right\} \cap$ $\left\{\left|[r-1, r] \cap Q_{t}\right|=1\right\}$. Note que $D \subset\{p(r)-p(0) \geq r+1\}$, portanto

$$
\mathbb{P}\left[Z_{t}-r \geq 1 \mid Z_{t-1}=r\right] \geq \mathbb{P}[D]=e^{-11 \kappa}(\kappa)^{2} e^{-2 \kappa}=e^{-13 \kappa}(\kappa)^{2} \geq e^{-13} \alpha^{2} .
$$

De (5.18) e (5.20) obtemos,

$$
\sup _{r \geq 10} \mathbb{P}\left[\left(U_{t}(r), V_{t}(r)\right) \in A_{\epsilon} \cup B_{\epsilon}\right] \leq\left(1-e^{-\epsilon}\right)\left(1-e^{-5}\right)^{2}+\left[1-\left(1-e^{-5 \alpha}\right)^{2}\right]+\frac{2 \epsilon e^{13}}{\alpha^{2}} .
$$

Seja $\epsilon_{0}$ suficientemente pequeno tal que

$$
\left(1-e^{-\epsilon_{0}}\right)\left(1-e^{-5}\right)^{2}+\left[1-\left(1-e^{-5 \alpha}\right)^{2}\right]+\frac{2 \epsilon_{0} e^{13}}{\alpha^{2}}=c_{2}\left(\epsilon_{0}\right)<1
$$

Assim,

$$
\sup _{r \geq 10} \mathbb{P}\left[\left(U_{t}(r), V_{t}(r)\right) \in A_{\epsilon_{0}} \cup B_{\epsilon_{0}}\right] \leq c_{2}\left(\epsilon_{0}\right)
$$

Agora, para $r \geq 10$,

$$
\begin{aligned}
& \mathbb{E}\left[\mathbb{I}_{\left\{Z_{t}>0\right\}} e^{-\lambda S_{t}(r)}\right] \leq \mathbb{E}\left[e^{-\lambda S_{t}(r)}\right] \\
& \leq \mathbb{E}\left[e^{-\lambda S_{t}(r)} \mathbb{I}_{\left\{\left(U_{t}, V_{t}\right) \in A_{\epsilon_{0}} \cup B_{\epsilon_{0}}\right\}}\right]+\mathbb{E}\left[e^{-\lambda S_{t}(r)} \mathbb{I}_{\left\{\left(U_{t}, V_{t}\right) \in\left(A_{\epsilon_{0}} \cup B_{\epsilon_{0}}\right)^{c}\right\}}\right] \\
& =\mathbb{E}\left[\mathbb{E}\left(e^{-\lambda S_{t}(r)} \mathbb{I}_{\left\{\left(U_{t}, V_{t}\right) \in A_{\epsilon_{0}} \cup B_{\epsilon_{0}}\right\}} \mid\left(U_{t}(r), V_{t}(r)\right)\right)\right]+\mathbb{E}\left[\mathbb{E}\left(e^{-\lambda S_{t}(r)} \mathbb{I}_{\left\{\left(U_{t}, V_{t}\right) \in\left(A_{\epsilon_{0}} \cup B_{\epsilon_{0}}\right)^{c}\right\}} \mid\left(U_{t}(r), V_{t}(r)\right)\right)\right] \\
& \leq \mathbb{P}\left[\left(U_{t}(r), V_{t}(r)\right) \in A_{\epsilon_{0}} \cup B_{\epsilon_{0}}\right]+\mathbb{E}\left[\mathbb{E}\left(e^{-\lambda S_{t}(r)} \mathbb{I}_{\left\{\left(U_{t}, V_{t}\right) \in\left[-r,-\epsilon_{0}\right) \times\left[\epsilon_{0}, \infty\right)\right\}} \mid\left(U_{t}(r), V_{t}(r)\right)\right)\right] .
\end{aligned}
$$

Dado $\left(U_{t}, V_{t}\right), S_{t}(r)$ é o tempo de parada de $B$ definido por $\left(U_{t}(r), V_{t}(r)\right)$, portanto se 
$T_{\epsilon_{0}}:=\inf \left\{t>0: B[t] \notin\left(-\epsilon_{0}, \epsilon_{0}\right)\right\}$ de [6] pág 400, temos que

$$
\begin{gathered}
\mathbb{E}\left[e^{-\lambda S_{t}(r)}\right] \leq \mathbb{P}\left[\left(U_{t}(r), V_{t}(r)\right) \in A_{\epsilon_{0}} \cup B_{\epsilon_{0}}\right]+\mathbb{E}\left[e^{-\lambda T_{\epsilon_{0}}}\right] \mathbb{P}\left[\left(U_{t}(r), V_{t}(r)\right) \in\left(A_{\epsilon_{0}} \cup B_{\epsilon_{0}}\right)^{c}\right] \\
\leq \mathbb{P}\left[\left(U_{t}(r), V_{t}(r)\right) \in A_{\epsilon_{0}} \cup B_{\epsilon_{0}}\right]+\frac{1}{\cosh \left(\sqrt{2 \lambda} \epsilon_{0}\right)}\left[1-\mathbb{P}\left[\left(U_{t}(r), V_{t}(r)\right) \in A_{\epsilon_{0}} \cup B_{\epsilon_{0}}\right]\right] . \\
=\mathbb{P}\left[\left(U_{t}(r), V_{t}(r)\right) \in A_{\epsilon_{0}} \cup B_{\epsilon_{0}}\right]\left(1-\frac{1}{\cosh \left(\sqrt{2 \lambda} \epsilon_{0}\right)}\right)+\frac{1}{\cosh \left(\sqrt{2 \lambda} \epsilon_{0}\right)} .
\end{gathered}
$$

Logo de (5.21) obtemos

$$
\begin{aligned}
\sup _{r \geq 10} \mathbb{E}\left[e^{-\lambda S_{t}(r)}\right] & \leq \sup _{r \geq 10} \mathbb{P}\left[\left(U_{t}(r), V_{t}(r)\right) \in A_{\epsilon_{0}} \cup B_{\epsilon_{0}}\right]\left(1-\frac{1}{\cosh \left(\sqrt{2 \lambda} \epsilon_{0}\right)}\right)+\frac{1}{\cosh \left(\sqrt{2 \lambda} \epsilon_{0}\right)} \\
& \leq c_{1}\left(\epsilon_{0}\right)\left(1-\frac{1}{\cosh \left(\sqrt{2 \lambda} \epsilon_{0}\right)}\right)+\frac{1}{\cosh \left(\sqrt{2 \lambda} \epsilon_{0}\right)} .
\end{aligned}
$$

Seja $c_{3}:=c_{3}\left(\epsilon_{0}\right)=c_{2}\left(\epsilon_{0}\right)\left(1-\frac{1}{\cosh \left(\sqrt{2 \lambda} \epsilon_{0}\right)}\right)+\frac{1}{\cosh \left(\sqrt{2 \lambda} \epsilon_{0}\right)}<1$ e seja $c_{4}=c_{4}\left(\epsilon_{0}\right)=c_{3}\left(\epsilon_{0}\right) \vee c_{1}<1$. Então de (5.22) e (5.10) obtemos

$$
\begin{gathered}
\sup _{r \leq 10} \mathbb{E}\left[\mathbb{I}_{\left\{Z_{t}>0\right\}} e^{-\lambda S_{t}\left(Z_{t-1}\right)} \mid Z_{t-1}=r\right] \leq c_{1} \quad \mathrm{e} \\
\sup _{r \geq 10} \mathbb{E}\left[\mathbb{I}_{\left\{Z_{t}>0\right\}} e^{-\lambda S_{t}\left(Z_{t-1}\right)} \mid Z_{t-1}=r\right] \leq c_{3} .
\end{gathered}
$$

Assim,

$$
\sup _{r>0} \mathbb{E}\left[\mathbb{I}_{\left\{Z_{t}>0\right\}} e^{-\lambda S_{t}\left(Z_{t-1}\right)} \mid Z_{t-1}=r\right] \leq c_{4}<1 .
$$

Note que $c_{4} \in(0,1)$ unicamente depende de $\epsilon_{0}$, por tanto é claro que:

$$
\max _{1 \leq i \leq t} \sup _{r>0} \mathbb{E}\left[\mathbb{I}_{\left\{Z_{i}>0\right\}} e^{-\lambda S_{i}\left(Z_{i-1}\right)} \mid Z_{i-1}=r\right]<c_{4}
$$

Então de (5.7) e (5.8) segue que

$$
\mathbb{P}[\tau>t] \leq \frac{c_{0}(\varsigma)}{\sqrt{t}}+\left[e^{\lambda \varsigma} c_{4}\right]^{t}
$$

Seja $\varsigma_{0}>0$ tal que $e^{\lambda_{\varsigma_{0}}} c_{4}=c_{5}<1$. Note que $c_{5}$ depende de $\epsilon_{0}, \varsigma_{0}$ unicamente, logo

$$
\mathbb{P}[\tau>t] \leq \frac{c_{0}\left(\varsigma_{0}\right)}{\sqrt{t}}+\left(c_{5}\left(\epsilon_{0}, \varsigma_{0}\right)\right)^{t}
$$


Como $c_{5} \in(0,1)$, existe $c_{6}=c_{6}\left(\epsilon_{0}, \varsigma_{0}\right)$ tal que $\left(c_{5}\right)^{t} \leq \frac{c_{6}}{\sqrt{t}}$, então tomando $c_{7}=c_{7}\left(\epsilon_{0}, \varsigma_{0}\right)=c_{0} \vee c_{6}$, obtemos que

$$
\mathbb{P}[\tau>t] \leq \frac{c_{7}}{\sqrt{t}}
$$

Lema 5.1.1 $\forall k \in \mathbb{N}, \forall u, v \in \mathbb{R} \times\{k\}$, tal que $v_{1}-u_{1}=1$. Se $Z_{t}:=Z_{t}(u, v)=X_{k+t}^{v}-X_{t+k}^{u} e$ $\tau_{k}:=\min \left\{t>k: Z_{t}=0\right\}$, temos que existe uma constante positiva $C$, tal que

$$
\mathbb{P}\left[\tau_{k}>t\right] \leq \frac{C}{\sqrt{t}}
$$

Prova: Na proposição anterior mostramos que $\mathbb{P}\left[\tau_{0}>t\right] \leq \frac{C}{\sqrt{t}}$ onde $C$ era uma constante positiva que não dependia do nível 0 e tampouco do nível $t$. O lema segue do teorema anterior e do fato que as taxas dos processos envolvidos ficam em $[\alpha, 1]$.

Lema 5.1.2 Seja $u=(x, 0)$ e $v=(y, 0) \operatorname{com} x<y$. Então,

$$
\mathbb{P}\left[Z_{t}(u, v)>0\right] \leq \frac{C(y-x)}{\sqrt{t}}
$$

Prova: Se $y-x \leq 1$ o resultado segue do lema anterior. Seja $y-x>1$, note que

$$
\left\{Z_{t}(u, v)>0\right\} \subset\left\{\cup_{i=0}^{\lceil y\rceil-\lfloor x\rfloor-1}\left\{Z_{t}((\lfloor x\rfloor+i, 0),(\lfloor x\rfloor+i+1))>0\right\}\right\} .
$$

Portanto,

$$
\begin{aligned}
\mathbb{P}\left[Z_{t}(u, v)>0\right] & \leq \sum_{i=0}^{\lceil y\rceil-\lfloor x\rfloor-1} \mathbb{P}\left[Z_{t}((\lfloor x\rfloor+i, 0),(\lfloor x\rfloor+i+1))>0\right] \\
& =(\lceil y\rceil-\lfloor x\rfloor-1) \mathbb{P}\left[Z_{t}((0,0),(1,0))>0\right] \\
& \leq 2(y-x) \mathbb{P}\left[Z_{t}((0,0),(1,0))>0\right] .
\end{aligned}
$$

Onde usamos o fato da invariância por translação dos processos de Poisson para obter a igualdade acima. Do lema anterior segue o resultado. 
Da observação 4.5.1, nosso objetivo final é mostrar a convergência em distribuição à Teia Browniana de $\hat{Y}^{n, T}$. Lembremos que as trajetórias de $\hat{Y}^{n, T}$ tem tempos de salto não homogêneos e os incrementos das trajetórias por nível são independentes mais não i.d, isto explica de alguma forma porque todas as nossas limitantes tem sido uniformes, isto é, não dependem do nível pois no futuro será fortemente usado.

\subsection{Condição (I)}

As ideias para mostrar que a familia de trajetórias $\hat{Y}^{n, T}$ satisfaz a condição $(I)$ do Teorema 2.2.2 são basicamente as mesmas ideias em [2], existen diferencias de tipo tecnico devido a que os tempos de salto não são homogêneos, as trajetórias estão restringidas num retângulo e além disso, as distribuições dos incrementos por nível são independentes mas não identicamente distribuidas.

Teorema 5.2.1 Sejam $\left(y_{0}, s_{0}\right),\left(y_{1}, s_{1}\right), \ldots,\left(y_{k}, s_{k}\right) k+1$ pontos diferentes em $\mathbb{R} \times[0,1 / \alpha-1)$ tais que $s_{0} \leq s_{1} \leq \ldots \leq s_{k}$ e se $s_{i-1}=s_{i}$ para algum $i, i=1, \ldots, k$, então $y_{i-1}<y_{i}$. Então,

$$
\left\{\hat{Y}_{\left(y_{0}, s_{0}\right)}^{n, T}, i=0, \ldots, k\right\} \underset{n \rightarrow \infty}{\stackrel{\mathcal{D}}{\longrightarrow}}\left\{W^{(i)}, i=0,1, \ldots, k\right\}
$$

onde $W^{(i)}$ são movimentos Brownianos caolescentes com constante de difusão positiva $\sqrt{2 \mathbb{E}\left[\left(\arg \min _{x \in P_{0}}|x|\right)^{2}\right]}$, e començando em $\left\{\left(y_{0}, s_{0}\right),\left(y_{1}, s_{1}\right), \ldots,\left(y_{k}, s_{k}\right)\right\}$ restritos ao conjunto $\mathbb{R} \times$ $[0,1 / \alpha-1]$.

Observação 5.2.1 Como um abuso de notação escrevemos $\hat{Y}_{\left(y_{0}, s_{0}\right)}^{n, T}$, pois o ponto $\left(y_{0}, s_{0}\right)$ não pertence (em geral) a algum nível do processo, mais dado que $\hat{Y}^{n, T} \stackrel{\mathcal{D}}{=} Y^{n}$ e o Lema 4.5.1, isto não representa problema.

A demonstração é dividida em três passos.

\section{Passo I.}

Para $k=0$ o resultado segue do Lema 4.5.1.

\section{Paso II}

Vamos considerar o caso $k \geq 1$ e $s_{0}=s_{1}=\ldots=s_{k}$ e $y_{i-1}<y_{i}$ para todo $i=1,2, \ldots, k$. 
Provaremos o caso $s_{0}=0$, pois a prova é análoga para qualquer ponto de $[0,1 / \alpha-1)$.

Para $k=1$, pela invariância por translação dos processos de Poisson é suficiente considerar o caso de dois trajetórias começando de $(0,0)$ e $\left(y_{1}, 0\right)$.

Vamos supor sem perda de generalidade que estamos trabalhando num espaço de probabilidade tal que

$$
\hat{Y}_{\left(y_{1}, 0\right)}^{(n, T)} \underset{n \rightarrow \infty}{\stackrel{q . c}{\longrightarrow}} W^{(1)}
$$

A seguinte proposição implica o resultado no caso $k=1$ e é a ferramenta principal para os outros casos.

Proposição 5.2.1 A distribuição condicional de $\hat{Y}_{(0,0)}^{n, T}$ dado $\hat{Y}_{\left(y_{1}, 0\right)}^{n, T}$ converge quase certamente à distribuição de $W^{(0)}$ dado $W^{(1)}$.

Sejam

$$
K_{n}^{(i)}=\left\{\left(\sqrt{n} x_{1}, n x_{2}\right):\left(x_{1}, x_{2}\right) \in \hat{Y}_{\left(y_{i}, 0\right)}^{n, T}\right\}, \quad i=0,1
$$

Portanto,

$$
K_{n}^{(i)}\left(l_{j}\right)=\sqrt{n} y_{i}+\sum_{h=1}^{j} S_{h}^{(i, n)}, 1 \leq j \leq k_{n}
$$

para $i=0,1$. Onde $S_{h}^{i, n}, 0 \leq h \leq k_{n}+1$ sao independentes e $S_{h}^{(i, n)} \stackrel{\mathcal{D}}{=} \arg \min _{x \in Q_{h}}|x| . \quad\left(\left(Q_{h}\right)_{h=0}^{k_{n}}\right.$ sao processos de Poisson em $\mathbb{R}$ independentes de taxa $\frac{n-h}{n}$ ).

A idéia da prova em [2] é aproximar $K_{n}{ }^{(0)}$ e $K_{n}{ }^{(1)}$ antes que eles estejam perto (uma distancia da ordem de $\left.n^{\gamma}, \gamma<1 / 2\right)$ por caminhos independentes e mostrar que depois daquele evento eles coalescem rapidamente. A nossa idéia varia um pouco devido a estarmos trabalhando em $[0, n(1 / \alpha-1)]$, a idéia é a seguinte:

Aproximar $K_{n}{ }^{(0)}$ e $K_{n}{ }^{(1)}$ antes que eles estejam perto (uma distancia da ordem de $n^{\gamma}, \gamma<1 / 2$ ) por caminhos independentes. Se o tempo em que eles estejam perto satisfaz que e menor que $n(1 / \alpha-1)-n^{\beta}, \operatorname{com} \beta(\gamma)<1$ então eles coalescem rapidamente, se o tempo é maior então a escala difusiva resolve o problema.

Consideremos agora copias independentes de $S_{h}^{(i, n)}$ para $i=0,1$. Sejam $\left\{\tilde{S}_{h}^{(i, n)}, 0 \leq h \leq k_{n}, i=0,1\right\}$ variáveis aleatórias independentes tais que $\tilde{S}_{h}^{(i, n)} \stackrel{\mathcal{D}}{=} \arg \min _{x \in Q_{h}}|x|$.

Seja $\gamma \in(0,1 / 2)$ fixo, lembrando que $l_{k}=\frac{n k}{n-k}$ para $k=0,1, \ldots, k_{n}+1$ definimos as seguintes 
variáveis aleatórias:

$$
\hat{S}_{h}^{(i, n)}= \begin{cases}S_{h}^{(i, n)}, & \text { se }\left|S_{h}^{(i, n)}\right| \leq n^{\gamma} \\ \tilde{S}^{(i, n)}, & \text { caso contrario }\end{cases}
$$

Definamos agora, $\hat{K}_{n}^{(i)}, i=0,1$ como o processo que interpola os seguintes pontos usando a a transformação $T$,

$$
\hat{K}_{n}^{(i)}\left(l_{k}\right)=\sqrt{n} y_{i}+\sum_{h=1}^{k} \hat{S}_{h}^{(i, n)} .
$$

$k=1,2, \ldots, k_{n}+1$, restritos ao conjunto $[0, n(1 / \alpha-1)]$.

Consideremos

$$
\hat{\tau}_{n}=\min \left\{l_{k}: \hat{K}_{n}^{(1)}\left(l_{k}\right)-\hat{K}_{n}^{(1)}\left(l_{k}\right) \leq 3 n^{\gamma}\right\} \wedge k_{n}
$$

e

$$
\tau_{n}=\min \left\{l_{k}: K_{n}^{(1)}\left(l_{k}\right)-K_{n}^{(1)}\left(l_{k}\right) \leq 3 n^{\gamma}\right\} \wedge k_{n}
$$

e seja $\tilde{K}_{n}^{(i)}, i=0,1$ o processo que interpola os seguintes pontos usando a transformação $T$,

$$
\tilde{K}_{n}^{(i)}\left(l_{k}\right)= \begin{cases}\hat{K}_{n}^{(i)}\left(l_{k}\right), & \text { se }\left(l_{k} \leq \hat{\tau}_{n}\right) \\ \hat{K}_{n}^{(i)}\left(\hat{\tau}_{n}\right)+\sum_{h=g\left(\hat{\tau}_{n}\right)+1}^{k} \breve{S}_{h}^{(i, n)}, & \text { caso contrário }\end{cases}
$$

$k=1,2, \ldots, k_{n}+1$, restritos ao conjunto $[0, n(1 / \alpha-1)]$ onde $g\left(\hat{\tau}_{n}\right)=\frac{n \hat{\tau}_{n}}{n+\hat{\tau}_{n}}$ e

$$
\breve{S}_{h}^{(i, n)}= \begin{cases}\tilde{S}_{h}^{(0, n)}, & \text { se } i=0 \\ S_{h}^{(0, n)}, & \text { se } i=1\end{cases}
$$

Lema 5.2.1 Seja $A_{n}:=\left\{S_{h}^{(i, n)}=\hat{S}_{h}^{(i, n)}, h=1,2, \ldots, k_{n}, i=0,1\right\}$ então

$$
\mathbb{P}\left[A_{n}^{c} \quad i . v\right]=0 .
$$

Prova do Lema 5.2.1

$$
\mathbb{P}\left[A_{n}^{c}\right] \leq \sum_{i=0}^{1} \sum_{k=1}^{k_{n}} \mathbb{P}\left[S_{k}^{(i, n)} \neq \hat{S}_{k}^{(i, n)}\right]
$$




$$
=2 \sum_{k=1}^{k_{n}} \mathbb{P}\left[\left|S_{k}^{(1, n)}\right|>n^{\gamma}\right]=2 \sum_{k=1}^{k_{n}} e^{-\frac{n-k}{n} n^{\gamma}} \leq 2 n e^{-\alpha n^{\gamma}} .
$$

O Lema segue de Borel-Cantelli.

Corolário 5.2.1 Considere o evento $B_{n}:=\left\{K_{n}^{(i)}\left(l_{k}\right)=\hat{K}_{n}^{(i)}\left(l_{k}\right) ; k=1,2, \ldots, k_{n} ; i=0,1\right\}$ então $\mathbb{P}\left[\begin{array}{ll}B_{n}^{c} & i . v\end{array}\right]=0$.

Prova do Corolário 5.2.1. Imediata do lema anterior e (5.30).

Observação 5.2.2 Da construção dos processos temos que $\tilde{K}_{n}^{(0)}$ e $\tilde{K}_{n}^{(1)}$ são processos independentes e $\tilde{K}_{n}^{(i)} \stackrel{\mathcal{D}}{=} K_{n}^{(i)}, i=0,1$.

Sejam $\beta \in(2 \gamma, 1)$ e $l_{n}^{\beta}:=\min \left\{l_{k}: l_{k} \geq l_{k_{n}}-n^{\beta}\right\}$. Isto é, $l_{n}^{\beta}$ é o primeiro nível dos processos cuja distancia até o nível final é menor que $n^{\beta}$.

Lema 5.2.2 $\mathbb{P}\left[K_{n}^{(1)}(s)>K_{n}^{(0)}(s), \tau_{n} \leq s \leq \tau_{n}+n^{\beta},\left\{\tau_{n} \leq l_{n}^{\beta}\right\}\right] \underset{n \rightarrow \infty}{\longrightarrow} 0$

Prova do Lema 5.2.2. Seja $k_{n}^{\beta} \in \mathbb{N}$ tal que $l_{n}^{\beta}=\frac{n k_{n}^{\beta}}{n-k_{n}^{\beta}}$ isto é $k_{n}^{\beta}=\frac{n l_{n}^{\beta}}{n+l_{n}^{\beta}}$. Portanto,

$$
\begin{gathered}
\mathbb{P}\left[K_{n}^{(1)}(s)>K_{n}^{(0)}(s), \tau_{n} \leq s \leq \tau_{n}+n^{\beta},\left\{\tau_{n} \leq l_{n}^{\beta}\right\}\right] \\
=\sum_{j=1}^{k_{n}^{\beta}} \mathbb{P}\left[K_{n}^{(1)}(s)>K_{n}^{(0)}(s), \tau_{n} \leq s \leq \tau_{n}+n^{\beta} ; \tau_{n}=l_{j}\right] \\
\leq \sum_{j=1}^{k_{n}^{\beta}} \mathbb{P}\left[K_{n}^{(1)}(s)>K_{n}^{(0)}(s), \tau_{n} \leq s \leq \tau_{n}+n^{\beta} \mid \tau_{n}=l_{j}\right] \mathbb{P}\left[\tau_{n}=l_{j}\right]
\end{gathered}
$$

É fácil ver que existe $C(\alpha)>1$ tal que $\forall k, k=0,1, \ldots, k_{n}$ temos que

$$
1 \leq l_{k+1}-l_{k} \leq C(\alpha)
$$

Além disso, $\mathbb{P}\left[K_{n}^{(1)}(s)>K_{n}^{(0)}(s), \tau_{n} \leq s \leq \tau_{n}+n^{\beta} \mid \tau_{n}=l_{j}\right]$ esta limitada superiormente pela probabilidade de ter dois pontos fixos a uma distancia $\left\lceil 3 n^{\gamma}\right\rceil$ no nível $l_{j}$ e não coalescer depois de um tempo $n^{\beta}$. De (5.34) temos que o processo deve percorrer pelo menos $\left\lfloor C(\alpha)^{-1} n^{\beta}\right\rfloor$ níveis sem ter coalescência. Portanto de maneira análoga ao Lema 5.1.2 obtemos que: 


$$
\mathbb{P}\left[K_{n}^{(1)}(s)>K_{n}^{(0)}(s), \tau_{n} \leq s \leq \tau_{n}+n^{\beta} \mid \tau_{n}=l_{j}\right] \leq C \frac{\left\lceil 3 n^{\gamma}\right\rceil}{\sqrt{\left\lfloor C(\alpha)^{-1} n^{\beta}\right\rfloor}}
$$

Agora, esta limitante é válida para todo $j=1,2, \ldots, k_{n}^{\beta}$. Portanto,

$$
\mathbb{P}\left[K_{n}^{(1)}(s)>K_{n}^{(0)}(s), \tau_{n} \leq s \leq \tau_{n}+n^{\beta},\left\{\tau_{n} \leq l_{n}^{\beta}\right\}\right] \leq C \frac{\left\lceil 3 n^{\gamma}\right\rceil}{\sqrt{\left\lfloor C(\alpha)^{-1} n^{\beta}\right\rfloor}} .
$$

Como $\beta \in(2 \gamma, 1)$ segue o lema.

Corolário 5.2.2 Sejam $\tilde{Z}_{n}^{(i)}:=\left\{\left(\frac{x_{1}}{\sqrt{n}}, \frac{x_{2}}{n}\right):\left(x_{1}, x_{2}\right) \in \tilde{K}_{n}^{(i)}\right\}$ então

1. $\tilde{Z}_{n}^{(1)} \underset{n \rightarrow \infty}{\stackrel{q . c}{\longrightarrow}} W^{(1)}$

2. $\left(\tilde{Z}_{n}^{(0)}, \tilde{Z}_{n}^{(1)}\right) \underset{n \rightarrow \infty}{\stackrel{\mathcal{D}}{\longrightarrow}}\left(\tilde{W}^{(0)}, W^{(1)}\right)$ onde $\tilde{W}^{(0)} \stackrel{\mathcal{D}}{=} W^{(0)}$, e $\tilde{W}^{(0)}$ e $W^{(1)}$ são independentes.

3. A distribuição condicional de $\left\{\tilde{Z}_{n}^{(0)}(t), t \leq \frac{\hat{\tau}_{n}}{n}\right\}$ dado $\tilde{Z}_{n}^{(1)}$ converge quase certamente $\grave{a}\left\{\tilde{W}^{(0)}(t), t \leq \tau\right\}$ dado $W^{(1)}$, onde $\tau=\inf \left\{t \geq 0: \tilde{W}^{(0)}(t)=W^{(1)}(t)\right\} \wedge\{1 / \alpha-1\}$.

\section{Prova do Corolário 5.2.2.}

1. segue imediatamente de (5.27), (5.28) e o Corolário 5.2.1.

2. segue do ponto 1, da Observação 5.2.2 e do Lema 4.5.1.

3. Seja $\bar{Z}_{n}^{(0)}$, tal que $\bar{Z}_{n}^{(0)} \stackrel{\mathcal{D}}{=} \tilde{Z}_{n}^{(1)}$ e

$$
\left(\bar{Z}_{n}^{(0)}, \tilde{Z}_{n}^{(1)}\right) \underset{n \rightarrow \infty}{\stackrel{q \cdot c}{\longrightarrow}}\left(\bar{W}^{(0)}, W^{(1)}\right)
$$

onde $\left(\bar{W}^{(0)}, W^{(1)}\right) \stackrel{\mathcal{D}}{=}\left(\tilde{W}^{(0)}, W^{(1)}\right)$. Definimos,

$$
\bar{\tau}_{n}:=\min \left\{l_{k} / n: \tilde{Z}_{n}^{(1)}\left(l_{k} / n\right)-\tilde{Z}_{n}^{(0)}\left(l_{k} / n\right) \leq 3 n^{\gamma-1 / 2}\right\} \wedge\{1 / \alpha-1\}
$$

e

$$
\bar{\tau}:=\inf \left\{t \geq 0: \bar{W}^{(0)}(t)=W^{(1)}(t)\right\} \wedge\{1 / \alpha-1\}
$$

É suficiente mostrar que $\bar{\tau}_{n} \underset{n \rightarrow \infty}{\stackrel{q . c}{\rightarrow}} \tau$. Isto segue de:

- $\mathbb{P}\left[\inf \left\{t \geq 0: \bar{W}^{(0)}=W^{(1)}(t)\right\}=1 / \alpha-1\right]=0$ (imediato), 
- $\mathbb{P}\left[\forall \epsilon>0, \bar{W}^{(0)}(t)>W^{(1)}(t)\right.$ para algum $\left.t \leq \bar{\tau}+\epsilon \mid \bar{\tau}<1 / \alpha-1\right]=1$ ( pela propriedade forte de Markov, a indepêndencia dos processos junto com a Proposição B.0.3).

e o seguinte resultado determinístico

Resultado. Sejam $f_{n}, g_{n}, f, g$ funções de $[0,1 / \alpha-1)$ em $\mathbb{R}$ continuas tais que $f(0)<g(0)$ e $T=\inf \{t \geq 0: f(t)=g(t)\} \in(0,1 / \alpha-1)$ e vale que $\forall \delta>0$, existe $t \in[T, T+\delta] \cap[0,1 / \alpha-1]$ com $f(t)>g(t)$. Também temos que

$$
\sup _{0 \leq t \leq(T+1) \wedge\{1 / \alpha-1\}}\left|f_{n}(t)-f(t)\right| \underset{n \rightarrow \infty}{\longrightarrow} 0 \quad \text { e } \quad \sup _{0 \leq t \leq(T+1) \wedge\{1 / \alpha-1\}}\left|g_{n}(t)-g(t)\right| \underset{n \rightarrow \infty}{\longrightarrow} 0 .
$$

Sejam $T_{n}=\inf \left\{t \geq 0: g_{n}(t)-f_{n}(t) \leq a_{n}\right\}$ donde $a_{n} \geq 0$ e $a_{n} \underset{n \rightarrow \infty}{\longrightarrow} 0$. Então, $T_{n} \underset{n \rightarrow \infty}{\longrightarrow} T$.

Definimos $Z_{n}^{(i)}:=\left\{\left(\frac{x_{1}}{\sqrt{n}}, \frac{x_{2}}{n}\right):\left(x_{1}, x_{2}\right) \in K_{n}^{(i)}\right\}=\tilde{Y}_{\left(y_{i}, 0\right)}^{n, T}, i=0,1$.

Corolário 5.2.3 A distribuição condicional de $\left\{Z_{n}^{(0)}(t), t \leq \frac{\tau_{n}}{n}\right\}$ dado $Z_{n}^{(1)}$ converge quase certamente à de $\left\{\tilde{W}^{(0)}, t \leq \tau\right\}$ dado $W^{(1)}$.

Prova do Corolário 5.2.3. segue do Corolário 5.2.1 e 3 do Corolário 5.2.2.

Corolário 5.2.4 $\left\{Z_{n}^{(0)}, t \geq \frac{\tau_{n}}{n}\right\}$ converge em probabilidade a $\left\{W^{(1)}(t), t \geq \tau\right\}$.

Prova: O Corolário 5.2.2 nos diz que uma vez os caminhos se encontram a uma distancia menor que $3 n^{\gamma}$ e o tempo em que acontece isto esta separado uma distância $n^{\beta} \operatorname{com} \beta \in(2 \gamma, 1)$ do ultimo nível, eles coalescem rapidamente. Agora para tempos a escala difusiva resolve o problema. Então é suficiente mostrar que $\forall \epsilon>0$,

$$
\mathbb{P}\left[\max _{0 \leq l \leq n^{\beta}}\left(K_{n}^{(1)}\left(l+\tau_{n}\right)-K_{n}^{(0)}\left(l+\tau_{n}\right)\right)>\epsilon \sqrt{n}\right] \underset{n \rightarrow \infty}{\longrightarrow} 0
$$

onde $\max _{0 \leq l \leq n^{\beta}}\left(K_{n}^{(1)}\left(l+\tau_{n}\right)-K_{n}^{(0)}\left(l+\tau_{n}\right)\right)=0$ se $l+\tau_{n}>k_{n}$.

Agora, dado $\tau_{n}=l_{j}$, o evento $\left\{\max _{0 \leq l \leq n^{\beta}}\left(K_{n}^{(1)}\left(l+\tau_{n}\right)-K_{n}^{(0)}\left(l+\tau_{n}\right)\right)>\epsilon \sqrt{n}\right\}$ é dominado estocasticamente pelos incrementos de um processo começa do nível $l_{j}$ cujos pontos iniciais tem uma distancia igual a $3 n^{\alpha}$ pela desigualdade de Doob para Martingais não negativas temos que:

$$
\mathbb{P}\left[\max _{0 \leq l \leq n^{\beta}}\left(K_{n}^{(1)}\left(l+\tau_{n}\right)-K_{n}^{(0)}\left(l+\tau_{n}\right)\right)>\epsilon \sqrt{n} \mid \tau_{n}=l_{j}\right] \leq \frac{3 n^{\gamma}}{\sqrt{\epsilon n}}
$$


Prova da Proposição 5.2.1. segue dos Corolários 5.2.3 e 5.2.4.

Consideremos agora o caso $k>1$. É suficiente mostrar que

$$
\mathbb{E}\left[f_{0}\left(Z_{n}^{(0)}\right) \ldots f_{k}\left(Z_{n}^{(k)}\right)\right] \underset{n \rightarrow \infty}{\longrightarrow} \mathbb{E}\left[f_{0}\left(W^{(0)}\right) \ldots f_{k}\left(W^{(k)}\right)\right]
$$

para todo $f_{0}, \ldots, f_{k} \in \mathcal{C}_{b}\left(\Pi_{0}^{1 / \alpha-1}, \mathbb{R}\right)$ o espaço de todas as funções continuas limitadas de $\Pi_{0}^{1 / \alpha-1} \mathrm{em}$ R. Logo,

$$
\begin{aligned}
\mathbb{E}\left[f_{0}\left(Z_{n}^{(0)}\right) \ldots f_{k}\left(Z_{n}^{(k)}\right)\right] & =\mathbb{E}\left\{f_{1}\left(Z_{n}^{(1)}\right) \mathbb{E}\left[f_{0}\left(Z_{n}^{(0)}\right) f_{2}\left(Z_{n}^{(2)}\right) \ldots f_{k}\left(Z_{n}^{(k)}\right) \mid Z_{n}^{(1)}\right]\right\} \\
& =\mathbb{E}\left\{f_{1}\left(Z_{n}^{(1)}\right) \mathbb{E}\left[f_{0}\left(Z_{n}^{(0)}\right) \mid Z_{n}^{(1)}\right] \mathbb{E}\left[f_{2}\left(Z_{n}^{(2)}\right) \ldots f_{k}\left(Z_{n}^{(k)}\right) \mid Z_{n}^{(1)}\right]\right\}
\end{aligned}
$$

pois dado $Z_{n}^{(1)}, Z_{n}^{(0)}$ e $\left(Z_{n}^{(2)}, \ldots, Z_{n}^{(k)}\right)$ são independentes. Como $Z_{n}^{(1)} \underset{n \rightarrow \infty}{\stackrel{q \cdot c}{\longrightarrow}} W^{(1)}$ então por indução temos que

$$
\mathbb{E}\left[f_{0}\left(Z_{n}^{(0)}\right) \mid Z_{n}^{(1)}\right] \underset{n \rightarrow \infty}{\stackrel{q \cdot c}{\rightarrow}} \mathbb{E}\left[f_{0}\left(W^{(0)}\right) \mid W^{(1)}\right]
$$

$\mathrm{e}$

$$
\mathbb{E}\left[f_{2}\left(Z_{n}^{(2)}\right) \ldots f_{k}\left(Z_{n}^{(k)}\right) \mid Z_{n}^{(1)}\right] \underset{n \rightarrow \infty}{\stackrel{q . c}{\longrightarrow}} \mathbb{E}\left[f_{2}\left(W^{(2)}\right) \ldots f_{k}\left(W^{(k)}\right) \mid W^{(1)}\right]
$$

Lembrando que as funções $f_{i}, i=0,1, \ldots, k$ são limitadas, pelo Teorema da convergência dominada temos que

$$
\begin{gathered}
\mathbb{E}\left\{f_{1}\left(Z_{n}^{(1)}\right) \mathbb{E}\left[f_{0}\left(Z_{n}^{(0)}\right) \mid Z_{n}^{(1)}\right] \mathbb{E}\left[f_{2}\left(Z_{n}^{(2)}\right) \ldots f_{k}\left(Z_{n}^{(k)}\right) \mid Z_{n}^{(1)}\right]\right\} \\
\underset{n \rightarrow \infty}{\longrightarrow} \mathbb{E}\left\{f_{1}\left(W^{(1)}\right) \mathbb{E}\left[f_{0}\left(W^{(0)}\right) \mid W^{(1)}\right] \mathbb{E}\left[f_{2}\left(W^{(2)}\right) \ldots f_{k}\left(W^{(k)}\right) \mid W^{(1)}\right]\right\} \\
=\mathbb{E}\left\{f_{1}\left(W^{(1)}\right) \mathbb{E}\left[f_{0}\left(W^{(0)}\right) f_{2}\left(W^{(2)}\right) \ldots f_{k}\left(W^{(k)}\right) \mid W^{(1)}\right]\right\} \\
=\mathbb{E}\left[f_{1}\left(W^{(1)}\right) f_{0}\left(W^{(0)}\right) f_{2}\left(W^{(2)}\right) \ldots f_{k}\left(W^{(k)}\right)\right] .
\end{gathered}
$$

Assim segue (5.37).

Paso III. Caso geral.

A prova é análoga a prova em [2] pag 14. 


\subsection{Condição $B_{1}$.}

Mostrar a condição $B_{1}$ do Teorema 2.2.2 para nosso modelo é equivalente a $\forall t \in(0,1 / \alpha-1)$,

$$
\limsup _{n \rightarrow \infty} \sup _{\left(a, t_{0}\right) \in \mathbb{R} \times[0,1 / \alpha-1]} \mathbb{P}\left[\eta_{\hat{Y}^{n, T}}\left(t_{0}, t ; a, a+\epsilon\right) \geq 2\right] \underset{\epsilon \rightarrow 0_{+}}{\longrightarrow} 0 .
$$

Seja $t \in(0,1 / \alpha-1)$ e $\epsilon>0$ fixos, seja $r_{n}(t):=\max \left\{k: \frac{k}{n-k}<1 / \alpha-1-t\right\}$ e $m_{k}=\frac{k}{n-k}$. Logo, pela invariância por translação no espaço (5.38) é equivalente a

$$
\limsup _{n \rightarrow \infty} \sup _{0 \leq k \leq r_{n}(t)} \mathbb{P}\left[\eta_{\hat{Y}^{n, T}}\left(m_{k}, t ; 0, \epsilon\right) \geq 2\right] \underset{\epsilon \rightarrow 0_{+}}{\longrightarrow} 0 .
$$

Seja $K^{n}:=\left\{\left(\sqrt{n} x_{1}, n x_{2}\right):\left(x_{1}, x_{2}\right) \in \hat{Y}^{n, T}\right\}$. segue que, para $k=0, \ldots, r_{n}(t)$

$$
\mathbb{P}\left[\eta_{\hat{Y}^{n, T}}\left(m_{k}, t ; 0, \epsilon\right) \geq 2\right]=\mathbb{P}\left[\eta_{K^{n}}\left(l_{k}, n t ; 0, \sqrt{n} \epsilon\right) \geq 2\right] .
$$

Note que como não há cruzamento de trajetórias o evento $\left\{\eta_{K^{n}}\left(l_{k}, n t ; 0, \sqrt{n} \epsilon\right) \geq 2\right\}$ é dominado pelo evento de duas trajetórias começando desde os pontos $\left(0, l_{k}\right)$ e $\left(\sqrt{n} \epsilon, l_{k}\right)$ visitar pelo menos $\left\lfloor C(\alpha)^{-1} n t\right\rfloor$ níveis e não coalescer, analogamente ao Lema 5.1 .2 , segue que para $k=0, \ldots, r_{n}(t)$

$$
\mathbb{P}\left[\eta_{K^{n}}\left(l_{k}, n t ; 0, \sqrt{n} \epsilon\right) \geq 2\right] \leq C \frac{\epsilon \sqrt{n}}{\sqrt{C(\alpha)^{-1} n t}}=C_{1}(\alpha) \frac{\epsilon}{\sqrt{t}}
$$

Isto implica (5.39).

\subsection{Condição $B_{2}$.}

Lema 5.4.1 Sejam $\epsilon>0$ fixo e Seja $A_{k}:=\left\{\mid Q_{k} \cap\left[0, \epsilon \sqrt{n}|| \in\left[\frac{\alpha}{2} \epsilon \sqrt{n}, \frac{2 e}{\alpha} \epsilon \sqrt{n}\right]\right\}\right.$ para $k=1,2, \ldots, k_{n}$. Então, $\lim \sup _{n \rightarrow \infty} \sup _{1 \leq k \leq k_{n}} \mathbb{P}\left[A_{k}^{c}\right]=0$.

Prova: Note que $A_{k}^{c}$ implica que $\left|Q_{k} \cap[0, \epsilon \sqrt{n}]\right|<\frac{\alpha}{2} \epsilon \sqrt{n}$ ou $\left|Q_{k} \cap[0, \epsilon \sqrt{n}]\right|>\frac{2 e}{\alpha} \epsilon \sqrt{n}$. Agora, $\left|Q_{k} \cap[0, \epsilon \sqrt{n}]\right|<\frac{\alpha}{2} \epsilon \sqrt{n}$ implica que o número de pontos do processo $Q_{k}$ em $[0, \epsilon \sqrt{n}]$ é menor do que $\frac{\alpha}{2} \epsilon \sqrt{n}$, isto é o ponto número $\left\lceil\frac{\alpha}{2} \epsilon \sqrt{n}\right\rceil$ é maior que $\epsilon \sqrt{n}$. Então,

$$
\mathbb{P}\left[\left|Q_{k} \cap[0, \epsilon \sqrt{n}]\right|<\frac{\alpha}{2} \epsilon \sqrt{n}\right] \leq \mathbb{P}\left[\sum_{i=1}^{\left\lceil\frac{\alpha}{2} \epsilon \sqrt{n}\right\rceil} T_{i}>\epsilon \sqrt{n}\right]
$$

onde $\left(T_{i}\right)_{i=1}^{\left\lceil\frac{\alpha}{2} \epsilon \sqrt{n}\right\rceil}$ são variáveis aleatórias i.i.d com $T_{1}$ exponencial de taxa $\frac{n-k}{n} \in[\alpha, 1]$. Portanto, 
pela lei dos grandes desvios $\mathbb{P}\left[\sum_{i=1}^{\left\lceil\frac{\alpha}{2} \epsilon \sqrt{n}\right\rceil} T_{i}>\epsilon \sqrt{n}\right]$ tem decaimento exponencialmente rápido para zero.

O evento $\left|Q_{k} \cap[0, \epsilon \sqrt{n}]\right|>\frac{2 e}{\alpha} \epsilon \sqrt{n}$ implica que a quantidade de pontos do processo em $[0, \epsilon \sqrt{n}]$ é maior do que $\frac{2 e}{\alpha} \epsilon \sqrt{n}$, portanto

$$
\mathbb{P}\left[\left|Q_{k} \cap[0, \epsilon \sqrt{n}]\right|>\frac{2 e}{\alpha} \epsilon \sqrt{n}\right] \leq \mathbb{P}\left[\sum_{i=1}^{\left\lceil\frac{2 e}{\alpha} \epsilon \sqrt{n}\right\rceil} T_{i}<\epsilon \sqrt{n}\right]
$$

Seja $M_{n}=\sum_{i=1}^{\left\lceil\frac{2 e}{\alpha} \epsilon \sqrt{n}\right\rceil} T_{i}$, segue que $M_{n}$ é uma variável aleatória Gamma com parâmetros $\left\lceil\frac{2 e}{\alpha} \epsilon \sqrt{n}\right\rceil$, $\frac{n}{n-k}$. Logo usando a formula de Stirling e a mesma idéia que na prova da propriedade 3 de $\xi^{n}$, obtemos que $\mathbb{P}\left[M_{n}<\epsilon \sqrt{n}\right]$ tem decaimento exponencialmente rápido para zero.

Do anterior concluímos que $\mathbb{P}\left[A_{k}^{c}\right]$ tem decaimento exponencialmente rápido para zero. Isto vale para todo $k=0,1, \ldots, k_{n}$ assim segue o lema.

Para mostrar a condição $B_{2}$ de Teorema 2.2.2 temos que provar que $\forall t \in(0,1 / \alpha-1)$,

$$
\epsilon^{-1} \limsup _{n \rightarrow \infty} \sup _{\left(a, t_{0}\right) \in \mathbb{R} \times[0,1 / \alpha-1]} \mathbb{P}\left[\eta_{\hat{Y}^{n, T}}\left(t_{0}, t ; a, a+\epsilon\right) \geq 3\right] \underset{\epsilon \rightarrow 0_{+}}{\longrightarrow} 0 .
$$

Isto é equivalente a

$$
\epsilon^{-1} \limsup _{n \rightarrow \infty} \sup _{0 \leq k \leq r_{n}(t)} \mathbb{P}\left[\eta_{\hat{Y}^{n, T}}\left(m_{k}, t ; 0, \epsilon\right) \geq 3\right] \underset{\epsilon \rightarrow 0_{+}}{\longrightarrow} 0 .
$$

segue que, para $k=0, \ldots, r_{n}(t)$

$$
\mathbb{P}\left[\eta_{\hat{Y}^{n, T}}\left(m_{k}, t ; 0, \epsilon\right) \geq 3\right]=\mathbb{P}\left[\eta_{K^{n}}\left(l_{k}, n t ; 0, \sqrt{n} \epsilon\right) \geq 3\right] .
$$

Dado $A_{0}=\left\{\left|Q_{0} \cap[0, \epsilon \sqrt{n}]\right|=m \in\left[\alpha / 2 \epsilon \sqrt{n}, \frac{2 e}{\alpha} \epsilon \sqrt{n}\right]\right\}$, sejam $x_{1}, x_{2}, \ldots, x_{m}$ os pontos do processo e seja

$$
\eta_{0}^{\prime}:=\eta_{0}^{\prime}\left(x_{1}, x_{2}, \ldots, x_{m}\right)=\left|\left\{K_{j}^{n}(t n): 1 \leq j \leq m\right\}\right|
$$

Logo,

$$
\mathbb{P}\left[\eta_{K^{n}}(0, n t ; 0, \sqrt{n} \epsilon) \geq 3 \mid A_{0}\right]=\mathbb{P}\left[\eta_{0}^{\prime} \geq 3\right]
$$

Agora o seguinte unicamente pode acrescentar a probabilidade, como $\frac{\epsilon \sqrt{n}}{m} \in\left[\frac{\alpha}{2 e}, \frac{2}{\alpha}\right]$, tomamos os pontos $y_{i}=i \frac{\epsilon \sqrt{n}}{m}$, para $i=0,1,2, \ldots, m$. Assim a distancia entre tais pontos não pode ser maior que $\frac{2}{\alpha}$, seja 


$$
\left\{z_{0}=0, z_{1}, z_{2}, \ldots, z_{2 m}, z_{2 m+1}=\epsilon \sqrt{n}\right\}
$$

o conjunto de pontos ordenado de menor a maior devido à união dos $x_{i}{ }^{\prime}$ s e $y_{i}{ }^{\prime}$ s. Seja $K_{j}$ a trajetória começãndo desde o ponto $z_{j}$.

Se $\eta_{0}^{\prime} \geq 3$, então existe um $1 \leq j \leq 2 m$ tal que $K_{j-1}(t n)<K_{j}(t n)<K_{2 m+1}(t n)$. Portanto do Lema B.0.4

$$
\begin{aligned}
\mathbb{P}\left[\eta_{0}^{\prime} \geq 3\right] & \leq \sum_{j=1}^{2 m} \mathbb{P}\left[K_{j-1}(t n)<K_{j}(t n)<K_{2 m+1}(t n)\right] \\
& =\lim _{r \rightarrow 0_{+}} \sum_{j=1}^{2 m} \mathbb{P}\left[\tilde{X}_{j-1}^{r}(t n)<\tilde{X}_{j}^{r}(t n)<\tilde{X}_{2 m+1}^{r}(t n)\right] \\
& =\lim _{r \rightarrow 0_{+}} \sum_{j=1}^{2 m} \int_{\tilde{\Pi}_{j}^{r}} \mathbb{P}\left[\tilde{X}_{j-1}^{r}(t n)<\tilde{X}_{j}^{r}(t n)<\tilde{X}_{2 m+1}^{r}(t n) \mid \tilde{X}_{j}^{r}=\pi\right] \mu_{\tilde{X}_{j}^{r}}(d \pi) \\
& =\lim _{r \rightarrow 0_{+}} \sum_{j=1}^{2 m} \int_{\tilde{\Pi}_{j}^{r}} \mathbb{P}\left[\tilde{X}_{j-1}^{r}(t n)<\tilde{X}_{j}^{r}(t n) \mid \tilde{X}_{j}^{r}=\pi\right] \mathbb{P}\left[\tilde{X}_{j}^{r}(t n)<\tilde{X}_{2 m+1}^{r}(t n) \mid \tilde{X}_{j}^{r}=\pi\right] \mu_{\tilde{X}_{j}^{r}}(d \pi)
\end{aligned}
$$

A ultima igualdade usamos o fato que dado $\tilde{X}_{j}^{r}=\pi, \tilde{X}_{j-1}^{r}(t n)<\tilde{X}_{j}^{r}(t n)$ e $\tilde{X}_{j}^{r}(t n)<\tilde{X}_{2 m+1}^{r}(t n)$ são independentes.

Agora na Proposição B.0.2 vimos que $\mathbb{P}\left[\tilde{X}_{j-1}^{r}(t n)<\tilde{X}_{j}^{r}(t n) \mid \tilde{X}_{j}^{r}=\pi\right]$ é crescente em $\pi$ e $\mathbb{P}\left[\tilde{X}_{j}^{r}(t n)<\right.$ $\left.\tilde{X}_{2 m+1}^{r}(t n) \mid \tilde{X}_{j}^{r}=\pi\right]$ é decrescente em $\pi$, logo usando a desigualdade FKG para $\mu_{\tilde{X}_{j}^{r}}(d \pi)$, obtemos que 


$$
\begin{aligned}
\mathbb{P}\left[\eta_{0}^{\prime} \geq 3\right] \leq & \lim _{r \rightarrow 0_{+}} \sum_{j=1}^{2 m} \int_{\tilde{\Pi}_{j}^{r}} \mathbb{P}\left[\tilde{X}_{j-1}^{r}(t n)<\tilde{X}_{j}^{r}(t n) \mid \tilde{X}_{j}^{r}=\pi\right] \mu_{\tilde{X}_{j}^{r}}(d \pi) \\
& \times \int_{\tilde{\Pi}_{j}^{r}} \mathbb{P}\left[\tilde{X}_{j}^{r}(t n)<\tilde{X}_{2 m+1}^{r}(t n) \mid \tilde{X}_{j}^{r}=\pi\right] \mu_{\tilde{X}_{j}^{r}}(d \pi) \\
= & \lim _{r \rightarrow 0_{+}} \sum_{j=1}^{2 m} \mathbb{P}\left[\tilde{X}_{j-1}^{r}(t n)<\tilde{X}_{j}^{r}(t n)\right] \mathbb{P}\left[\tilde{X}_{j}^{r}(t n)<\tilde{X}_{2 m+1}^{r}(t n)\right] \\
= & \sum_{j=1}^{2 m} \mathbb{P}\left[K_{j-1}(t n)<K_{j}(t n)\right] \mathbb{P}\left[K_{j}(t n)<K_{2 m+1}(t n)\right] \\
\leq & \sum_{j=1}^{2 m} \mathbb{P}\left[K_{j-1}(t n)<K_{j}(t n)\right] \mathbb{P}\left[K_{0}(t n)<K_{2 m+1}(t n)\right] \\
\leq & 2 m \mathbb{P}\left[K_{(0,0)}(t n)<K_{(2 / \alpha, 0)}\right] \mathbb{P}\left[K_{0}(t n)<K_{2 m+1}(t n)\right] \\
\leq & 4 \frac{e \epsilon}{\alpha} \sqrt{n} \mathbb{P}\left[K_{(0,0)}(t n)<K_{(2 / \alpha, 0)}(t n)\right] \mathbb{P}\left[K_{0}(t n)<K_{2 m+1}(t n)\right] .
\end{aligned}
$$

$K_{(0,0)}(t n)<K_{(2 / \alpha, 0)}(t n)$ implica que começãndo a uma distancia $2 / \alpha$ no nível $k=0$, depois de $C(\alpha)^{-1}$ tn níveis visitados não temos coalescencia, logo sabemos que

$$
\mathbb{P}\left[K_{(0,0)}(t n)<K_{(2 / \alpha, 0)}(t n)\right] \leq \frac{\lceil 2 / \alpha\rceil}{\sqrt{C(\alpha)^{-1} n t}}
$$

e analogamente

$$
\mathbb{P}\left[K_{0}(t n)<K_{2 m+1}(t n)\right] \leq \frac{\lceil\epsilon \sqrt{n}\rceil}{\sqrt{C(\alpha)^{-1} n t}}
$$

Assim, $\mathbb{P}\left[\eta_{0}^{\prime} \geq 3\right] \leq \tilde{C}(\alpha, t) \epsilon^{2}$, onde $\tilde{C}(\alpha, t)>0$. De (5.42), temos que

$$
\mathbb{P}\left[\eta_{K^{n}}(0, n t ; 0, \sqrt{n} \epsilon) \geq 3\right] \leq \tilde{C}(\alpha, t) \epsilon^{2}+\mathbb{P}\left[A_{0}^{c}\right] .
$$

Analogamente, mostramos que para $k=1,2, \ldots, r_{n}(t)$

$$
\mathbb{P}\left[\eta_{K^{n}}\left(l_{k}, n t ; 0, \sqrt{n} \epsilon\right) \geq 3\right] \leq \tilde{C}(\alpha, t) \epsilon^{2}+\mathbb{P}\left[A_{k}^{c}\right]
$$

Portanto,

$$
\sup _{0 \leq k \leq r_{n}(t)} \mathbb{P}\left[\eta_{K^{n}}\left(l_{k}, n t ; 0, \sqrt{n} \epsilon\right) \geq 3\right] \leq \tilde{C}(\alpha, t) \epsilon^{2}+\sup _{0 \leq k \leq r_{n}(t)} \mathbb{P}\left[A_{k}^{c}\right] .
$$

Do Lema 5.4.1, segue que

$$
\limsup _{n \rightarrow \infty} \sup _{0 \leq k \leq r_{n}(t)} \mathbb{P}\left[\eta_{K^{n}}\left(l_{k}, n t ; 0, \sqrt{n} \epsilon\right) \geq 3\right] \leq \tilde{C}(\alpha, t) \epsilon^{2}
$$


De (5.43) segue a condição $B_{2}$.

\subsection{Prova do Teorema principal}

Agora temos todas as ferramentas para provar o Teorema 3.3.1, ouseja

$$
\mathfrak{E}^{n}\left[\xi^{n}\right] \underset{n \rightarrow \infty}{\stackrel{\mathcal{D}}{\longrightarrow}} T^{-1}\left[\overline{\mathcal{W}}_{0,1 / \alpha-1}\right] .
$$

Lembremos que $\tilde{\xi}^{n}=\mathfrak{E}^{n}\left[\xi^{n}\right]$. Dos Lemas 4.1.3, 4.2.1 e 4.3.1 obtemos que

$$
d_{\mathcal{H}_{-1,-\alpha}}\left(\tilde{\xi}^{n}, \tilde{\xi}^{3, n}\right) \underset{n \rightarrow \infty}{\stackrel{p}{\longrightarrow}} 0 .
$$

Temos que $T\left[\tilde{\xi}^{3, n}\right]=Y^{n} \stackrel{\mathcal{D}}{=} \hat{Y}^{n, T}$ e que $\hat{Y}^{n, T}$ satisfaz as condições $(I),\left(B_{1}\right)$ e $\left(B_{2}\right)$ no Teorema 2.2.2, $\operatorname{assim} \hat{Y}^{n, T} \underset{n \rightarrow \infty}{\stackrel{\mathcal{D}}{\longrightarrow}} \overline{\mathcal{W}}_{0,1 / \alpha-1}$, logo (5.44) segue de (5.45) e o Teorema do mapeamento continuo. 


\section{Apêndice A}

\section{Convergência em distribuição}

Sejam $k>0$ fixo e $D=D_{[0, k]}$ o espaço das funções $x:[0, k] \mapsto \mathbb{R}$ continuas a direita e com limite a esquerda:

- Para $0 \leq t<k, x(t)=\lim _{s \rightarrow t^{+}} x(s) \mathrm{e}$

- Para $0<t \leq k, \lim _{s \rightarrow t^{-}} x(s)=x(t-)$ existe.

Seja $\Lambda^{\prime}$ a familia de funções continuas, estritamente crescentes $\lambda:[0, k] \mapsto[0, k]$ tais que $\lambda(0)=0$ e $\lambda(k)=k$. Seja $\Lambda$ o conjunto de funções Lipschitz $\lambda \in \Lambda^{\prime}$ tais que

$$
\gamma(\lambda) \equiv e s s \sup \left|\log \lambda^{\prime}(t)\right|=\sup _{s>t \geq 0}\left|\log \left(\frac{\lambda(s)-\lambda(t)}{s-t}\right)\right|<\infty .
$$

Para $x, y \in D_{[0, k]}$, define-se

$$
d(x, y)=\inf _{\lambda \in \Lambda}\left[\gamma(\lambda) \vee \sup _{0 \leq t \leq k}|x(t)-y(\lambda(t))|\right]
$$

Para medidas de probabilidade $\mathbb{P}$ sobre $(D, \mathfrak{D})$, ( $\mathfrak{D}$ é a $\sigma$-álgebra de Borel induzida por $d$ ) denotamos por $T_{\mathbb{P}}$ o conjunto de pontos $t$ em $[0, k]$ para os quais a projeção $\pi_{t}$ é continua salvo em um conjunto de pontos de $\mathbb{P}$-medida 0 .

Note que os pontos 0 e $k$ pertencem a $T_{\mathbb{P}}$.

Teorema A.0.1 Seja $\left(X^{n}\right)_{n=1}^{\infty}, X \in D_{[0, k]}$. Suponha que para todo subconjunto finito de pontos, $\left\{t_{1}, t_{2}, \ldots, t_{j}\right\} \subset T_{\mu_{X}}$, temos que

$$
\left(X_{t_{1}}^{n}, \ldots, X_{t_{j}}^{n}\right) \underset{n \rightarrow \infty}{\stackrel{\mathcal{D}}{\longrightarrow}}\left(X_{t_{1}}, \ldots, X_{t_{j}}\right),
$$


que

$$
X_{k}-X_{k-\delta} \underset{\delta \rightarrow 0}{\stackrel{\mathcal{D}}{\longrightarrow}} 0
$$

e que, para $r \leq s \leq t, n \geq 1, \beta \geq 0, \alpha>1 / 2$ e $F$ continua e não decrescente sobre $[0, k]$

$$
\mathbb{E}\left[\left|X_{t}^{n}-X_{s}^{n}\right|^{2 \beta}\left|X_{s}^{n}-X_{r}^{n}\right|^{2 \beta}\right] \leq[F(t)-F(r)]^{2 \alpha} .
$$

Então $X^{n} \underset{n \rightarrow \infty}{\stackrel{\mathcal{D}}{\longrightarrow}} X$ em $D_{[0, k]}$.

Prova: Ver [4] pág 142.

Proposição A.0.1 Para $n=1,2, \ldots$, seja $\left\{Y_{n}(k), k=0,1,2, \ldots\right\}$ um processo discreto que assume valores em $\mathbb{R}$, seja $\alpha_{n}>0$, e definimos

$$
X_{t}^{n}=Y_{n}\left(\left\lfloor\alpha_{n} t\right\rfloor\right)
$$

e

$$
Z_{t}^{n}=Y_{n}\left(\left\lfloor\alpha_{n} t\right\rfloor\right)+\left(\alpha_{n} t-\left\lfloor\alpha_{n} t\right\rfloor\right)\left(Y_{n}\left(\left\lfloor\alpha_{n} t\right\rfloor+1\right)-Y_{n}\left(\left\lfloor\alpha_{n} t\right\rfloor\right)\right)
$$

para todo $t \in[0, k]$. Note que $X^{n}$ tem trajetórias em $D_{[0, k]}$ e $Z^{n}$ tem trajetórias em $\mathcal{C}_{[0, k]}$. Se $\alpha_{n} \underset{n \rightarrow \infty}{\longrightarrow} \infty$ e $X$ é um processo com trajetórias em $\mathcal{C}_{[0, k]}$, então

$$
X^{n} \underset{n \rightarrow \infty}{\stackrel{\mathcal{D}}{\longrightarrow}} X \quad \text { em } \quad D_{[0, k]} \Leftrightarrow Z_{n} \underset{n \rightarrow \infty}{\stackrel{\mathcal{D}}{\rightarrow}} X \quad \text { em } \quad \mathcal{C}[0, k]
$$

Prova: Ver [5] pág 149.

Sejam $\alpha \in(0,1)$ fixo e seja $f(t)=(1-t)$, para $t \in[0,1-\alpha]$. Seja $B$ um movimento Browniano Standard começando em 0 . Considere o processo

$$
\left(B\left[t^{\prime}\right], t^{\prime}\right), \quad t^{\prime} \in\left[0, \frac{1}{\alpha}-1\right]
$$

Então

$$
\left(B\left[t^{\prime}\right], t^{\prime}\right)=\left(B\left[\frac{1}{f(t)}-1\right], \frac{1}{f(t)}-1\right), \quad 0 \leq t \leq 1-\alpha
$$

e considere o mapeamento continuo $T_{1}: \mathcal{C}_{[0,1 / \alpha-1]} \longrightarrow \mathcal{C}_{[0,1-\alpha]}$ tal que 


$$
\left(B\left[\frac{1}{f(t)}-1\right], \frac{1}{f(t)}-1\right) \stackrel{T_{1}}{\longmapsto}\left(c f(t) B\left[\frac{1}{f(t)}-1\right], t\right), \quad 0 \leq t \leq 1-\alpha .
$$

Com $c>0$. Seja $g(t)=\frac{1}{f(t)}-1$ e $X$ o processo definido por

$$
X_{t}=c f(t) B[g(t)], \quad t \in[0,1-\alpha]
$$

Observação A.0.1 O processo $X$ é obtido usando um mapeamento continuo de um movimento Browniano standard em $\mathcal{C}_{[0,1 / \alpha-1]}$.

Sejam $\left\{P_{j}\right\}_{j=1}^{\infty}$ uma sequência de processos de Poisson em $\mathbb{R}$ independentes de taxa 1.

Seja $\varepsilon_{j}=\arg \min _{x \in P_{j}}|x|$ e $k_{n}(\alpha)=k_{n}=\lfloor n(1-\alpha)\rfloor$.

Sejam $Y_{n}(0)=0$ e $Y_{n}(k)=f_{n}(k) \sum_{j=1}^{k} \frac{\sqrt{n} \varepsilon_{j}}{n-j}$, para $k=1,2, \ldots, k_{n}$, onde $f_{n}(k)=\frac{n-k}{n}$.

Seja

$$
X_{t}^{n}=Y_{n}(\lfloor n t\rfloor), 0 \leq t \leq 1-\alpha
$$

Lema A.0.1 Sejam $\left(X^{n}\right)$ como em (A.11), $X$ como em $(A .10)$ com $c=\sqrt{2 \mathbb{E}\left[\varepsilon_{1}^{2}\right]}$. Suponha que para todo subconjunto finito, $\left\{t_{1}, t_{2}, \ldots, t_{j}\right\} \subset[0,1-\alpha]$, temos que

$$
\left(X_{t_{1}}^{n}, \ldots, X_{t_{j}}^{n}\right) \underset{n \rightarrow \infty}{\stackrel{\mathcal{D}}{\longrightarrow}}\left(X_{t_{1}}, \ldots, X_{t_{j}}\right)
$$

Então, $X^{n} \underset{n \rightarrow \infty}{\stackrel{\mathcal{D}}{\longrightarrow}} X$ em $D_{[0,1-\alpha]}$.

Prova: Do Teorema A.0.1 temos que mostrar que

$$
X_{1-\alpha}-X_{1-\alpha-\delta} \underset{\delta \rightarrow 0}{\stackrel{\mathcal{D}}{\longrightarrow}} 0 .
$$

e que para $r \leq s \leq t, n \geq 1, \beta \geq 0, \gamma>1 / 2$ e $F$ continua e não decrescente sobre $[0,1-\alpha]$,

$$
\mathbb{E}\left[\left|X_{s}^{n}-X_{r}^{n}\right|^{2 \beta}\left|X_{t}^{n}-X_{s}^{n}\right|^{2 \beta}\right] \leq[F(t)-F(r)]^{2 \gamma} .
$$

(A.13) segue da observação A.0.1 e do fato que o movimento Browniano $B$ é continuo em 1/ $\alpha-1$.

Seja $\tilde{X}_{t}^{n}=\sum_{j=1}^{\lfloor n t\rfloor} \frac{\sqrt{n} \varepsilon_{j}}{n-j}$ para $t \in[0,1-\alpha]$, onde $\tilde{X}_{0}^{n}=0$. Assim, $X_{t}^{n}=f_{n}(\lfloor n t\rfloor) \tilde{X}_{t}^{n}$. Então 


$$
\begin{aligned}
\left|X_{s}^{n}-X_{r}^{n}\right|^{2} & =\left|f_{n}(\lfloor n s\rfloor) \tilde{X}_{s}^{n}-f_{n}(\lfloor n r\rfloor) \tilde{X}_{r}^{n}\right|^{2} \\
& =\left|f_{n}(\lfloor n s\rfloor) \tilde{X}_{s}^{n}-f_{n}(\lfloor n s\rfloor) \tilde{X}_{r}^{n}+f_{n}(\lfloor n s\rfloor) \tilde{X}_{r}^{n}-f_{n}(\lfloor n r\rfloor) \tilde{X}_{r}^{n}\right|^{2} \\
& =\left|f_{n}(\lfloor n s\rfloor)\left(\tilde{X}_{s}^{n}-\tilde{X}_{r}^{n}\right)+\tilde{X}_{r}^{n}\left(f_{n}(\lfloor n s\rfloor)-f_{n}(\lfloor n r\rfloor)\right)\right|^{2} \\
& \leq 2\left|f_{n}(\lfloor n s\rfloor)\right|^{2}\left|\tilde{X}_{s}^{n}-\tilde{X}_{r}^{n}\right|^{2}+2\left|\tilde{X}_{r}^{n}\right|^{2}\left|f_{n}(\lfloor n s\rfloor)-f_{n}(\lfloor n r\rfloor)\right|^{2} \\
& \leq 2\left|\tilde{X}_{s}^{n}-\tilde{X}_{r}^{n}\right|^{2}+2\left(\frac{\lfloor n s\rfloor-\lfloor n r\rfloor}{n}\right)^{2}\left|\tilde{X}_{r}^{n}\right|^{2} \\
& \leq 2\left|\tilde{X}_{s}^{n}-\tilde{X}_{r}^{n}\right|^{2}+2\left(\frac{\lfloor n t\rfloor-\lfloor n r\rfloor}{n}\right)^{2}\left|\tilde{X}_{r}^{n}\right|^{2}
\end{aligned}
$$

análogamente

$$
\left|X_{t}^{n}-X_{s}^{n}\right|^{2} \leq 2\left|\tilde{X}_{t}^{n}-\tilde{X}_{s}^{n}\right|^{2}+2\left(\frac{\lfloor n t\rfloor-\lfloor n r\rfloor}{n}\right)^{2}\left|\tilde{X}_{s}^{n}\right|^{2}
$$

Note que se $t-r \leq 1 / n$ então $\lfloor n s\rfloor=\lfloor n t\rfloor$ ou $\lfloor n s\rfloor=\lfloor n r\rfloor$ e portanto

$$
\mathbb{E}\left[\left|X_{s}^{n}-X_{r}^{n}\right|^{2}\left|X_{t}^{n}-X_{s}^{n}\right|^{2}\right]=0 .
$$

Suponhamos então que $t-r>1 / n$. De (A.15) e (A.16) temos que

$$
\left|X_{s}^{n}-X_{r}^{n}\right|^{2}|| X_{t}^{n}-\left.X_{s}^{n}\right|^{2} \leq(I)+(I I)+(I I I)+(I V),
$$

onde

$$
\begin{gathered}
(I)=4\left|\tilde{X}_{s}^{n}-\tilde{X}_{r}^{n}\right|^{2}\left|\tilde{X}_{t}^{n}-\tilde{X}_{s}^{n}\right|^{2}, \\
(I I)=4\left(\frac{\lfloor n t\rfloor-\lfloor n r\rfloor}{n}\right)^{2}\left|\tilde{X}_{s}^{n}-\tilde{X}_{r}^{n}\right|^{2}\left|\tilde{X}_{s}^{n}\right|^{2}, \\
(I I I)=4\left(\frac{\lfloor n t\rfloor-\lfloor n r\rfloor}{n}\right)^{2}\left|\tilde{X}_{t}^{n}-\tilde{X}_{s}^{n}\right|^{2}\left|\tilde{X}_{r}^{n}\right|^{2}
\end{gathered}
$$

e

$$
(I V)=4\left(\frac{\lfloor n t\rfloor-\lfloor n r\rfloor}{n}\right)^{4}\left|\tilde{X}_{r}^{n}\right|^{2}\left|\tilde{X}_{s}^{n}\right|^{2}
$$

Agora, por independência 


$$
\begin{aligned}
\mathbb{E}[(I)] & =4 \mathbb{E}\left[\left|\tilde{X}_{s}^{n}-\tilde{X}_{r}^{n}\right|^{2}\right] \mathbb{E}\left[\left|\tilde{X}_{t}^{n}-\tilde{X}_{s}^{n}\right|^{2}\right] \\
& \leq 4\left\{\sum_{j=\lfloor n r\rfloor+1}^{\lfloor n s\rfloor} \frac{n \mathbb{E}\left[\varepsilon_{1}^{2}\right]}{[n-j]^{2}}\right\} \times\left\{\sum_{j=\lfloor n s\rfloor+1}^{\lfloor n t\rfloor} \frac{n \mathbb{E}\left[\varepsilon_{1}^{2}\right]}{[n-j]^{2}}\right\} \\
& \leq 4\left(\mathbb{E}\left[\varepsilon_{1}^{2}\right]\right)^{2} \times \sum_{j=\lfloor n r\rfloor+1}^{\lfloor n s\rfloor} \frac{n}{(n \alpha)^{2}} \times \sum_{j=\lfloor n s\rfloor+1}^{\lfloor n t\rfloor} \frac{n}{(n \alpha)^{2}} \\
& \leq 4\left(\mathbb{E}\left[\varepsilon_{1}^{2}\right]\right)^{2} \alpha^{-4}\left(\frac{\lfloor n s\rfloor-\lfloor n r\rfloor}{n}\right)\left(\frac{\lfloor n t\rfloor-\lfloor n s\rfloor}{n}\right) \\
& \leq 4\left(\mathbb{E}\left[\varepsilon_{1}^{2}\right]\right)^{2} \alpha^{-4}\left(\frac{\lfloor n t\rfloor-\lfloor n r\rfloor}{n}\right)^{2}
\end{aligned}
$$

como $t-r>1 / n$, então

$$
\mathbb{E}[(I)] \leq 4\left(\mathbb{E}\left[\varepsilon_{1}^{2}\right]\right)^{2} \alpha^{-4} \times 4(t-r)^{2}=C_{1}(t-r)^{2} .
$$

Como $t-r>1 / n$ e da desigualdade de Hölder obtemos

$$
\begin{aligned}
\mathbb{E}[(I I)] & =4\left(\frac{\lfloor n t\rfloor-\rfloor n r\rfloor}{n}\right)^{2} \mathbb{E}\left[\left|\tilde{X}_{s}^{n}-\tilde{X}_{r}^{n}\right|^{2}\left|\tilde{X}_{s}^{n}\right|^{2}\right] \\
& \leq 16(t-r)^{2}\left\{\mathbb{E}\left[\left|\tilde{X}_{s}^{n}-\tilde{X}_{r}^{n}\right|^{4}\right]\right\}^{1 / 2}\left\{\mathbb{E}\left[\left|\tilde{X}_{s}^{n}\right|^{4}\right]\right\}^{1 / 2}
\end{aligned}
$$

agora,

$$
\begin{aligned}
\mathbb{E}\left[\left|\tilde{X}_{s}^{n}\right|^{4}\right] & \leq \sum_{j=1}^{\lfloor n s\rfloor} \frac{n^{2} \mathbb{E}\left[\varepsilon_{1}^{4}\right]}{[n-j]^{4}}+\sum_{\substack{i \neq j \\
1 \leq i, j \leq\lfloor n s\rfloor}} \frac{n^{2}\left(\mathbb{E}\left[\varepsilon_{1}^{2}\right]\right)^{2}}{[n-j]^{4}} \\
& \leq \frac{n^{3} \mathbb{E}\left[\varepsilon_{1}^{4}\right]}{(n \alpha)^{4}}+\frac{n^{4}\left(\mathbb{E}\left[\varepsilon_{1}^{2}\right]\right)^{2}}{(n \alpha)^{4}} \leq C_{2}
\end{aligned}
$$

onde $C_{2}>0$ constante que depende de $\alpha$ e de $\mathbb{E}\left[\varepsilon_{1}^{2}\right], \mathbb{E}\left[\varepsilon_{1}^{4}\right]$. Analogamente,

$$
\mathbb{E}\left[\left|\tilde{X}_{s}^{n}-\tilde{X}_{r}^{n}\right|^{4}\right] \leq C_{2}
$$

De (A.20), (A.21) e (A.22) temos que 


$$
\mathbb{E}[(I I)] \leq C_{3}(t-r)^{2}
$$

Como $t-r>1 / n$, analogamente

$$
\mathbb{E}[(I I I)] \leq C_{3}(t-r)^{2} .
$$

De novo, usando que $t-r<1 / n$, a desigualdade de Hölder e os mesmos argumentos anteriores obtemos

$$
\mathbb{E}[(I V)] \leq C_{4}(t-r)^{4}
$$

dado $|t-r| \leq 1$, então

$$
\mathbb{E}[(I V)] \leq C_{4}(t-r)^{2} .
$$

De (A.17), (A.18), (A.19) e (A.23), (A.24), (A.25) obtemos que

$$
\mathbb{E}\left[\left|X_{s}^{n}-X_{r}^{n}\right|^{2}\left|X_{t}^{n}-X_{s}^{n}\right|^{2}\right] \leq C_{5}(t-r)^{2},
$$

$\operatorname{com} \beta=1, \gamma=1$ e $F(t)=\sqrt{C_{5}} t$ segue o Lema.

Lema A.0.2 As distribuições finito dimensionais do processo $X^{n}$ em (A.11), convergem fracamente às distribuições finito dimensionais do processo $X$ em $(A .10)$, com $c=\sqrt{2 \mathbb{E}\left[\varepsilon_{1}^{2}\right]}$.

Prova: Seja $t \in(0,1-\alpha]$ fixo. Temos que $X_{t}^{n}=f_{n}(\lfloor n t\rfloor) \tilde{X}_{t}^{n}$, onde

$$
f_{n}(\lfloor n t\rfloor)=\frac{n-\lfloor n t\rfloor}{n} \quad \text { e } \quad \tilde{X}_{t}^{n}=\sum_{j=1}^{\lfloor n t\rfloor} \frac{\sqrt{n} \varepsilon_{j}}{n-j} .
$$

Sejam $Y_{n k}=\frac{\sqrt{n} \varepsilon_{k}}{n-k}$ para $k=1,2, \ldots, k_{n}, r_{n}=\lfloor n t\rfloor$. Logo note que,

$$
\tilde{X}_{t}^{n}=\sum_{j=1}^{r_{n}} Y_{n j}
$$

Então

$$
\mathbb{E}\left[Y_{n k}\right]=0, \quad \sigma_{n k}^{2}=\operatorname{Var}\left[Y_{n k}\right]=\frac{n \mathbb{E}\left[\left(\varepsilon_{1}\right)^{2}\right]}{[n-k]^{2}} \quad \text { e } \quad s_{n}^{2}=\sum_{j=1}^{r_{n}} \sigma_{n j}^{2}
$$

assim, $s_{n}^{2}=\sum_{j=1}^{r_{n}} \frac{n \mathbb{E}\left[\left(\varepsilon_{1}\right)^{2}\right]}{[n-j]^{2}} \geq t \mathbb{E}\left[\left(\varepsilon_{1}\right)^{2}\right]=C_{0}>0$. Então, seja $\epsilon>0$ fixo, 


$$
\begin{aligned}
\sum_{j=1}^{r_{n}} \frac{1}{s_{n}^{2}} \mathbb{E}\left[Y_{n k}^{2} \mathbb{I}_{\left\{\left|Y_{n k}\right| \geq s_{n} \epsilon\right\}}\right] & \leq C_{1} \sum_{j=1}^{r_{n}} \mathbb{E}\left[\frac{n\left(\varepsilon_{j}\right)^{2}}{[n-j]^{2}} \mathbb{I}_{\left\{\left|\varepsilon_{j}\right| \geq \frac{n-j}{\sqrt{n}} s_{n} \epsilon\right\}}\right] \quad\left(C_{1}=1 / C_{0}\right) \\
& \leq C_{1} \frac{n}{\left[n-r_{n}\right]^{2}} \sum_{j=1}^{r_{n}} \mathbb{E}\left[\left(\varepsilon_{j}\right)^{2} \mathbb{I}_{\left\{\left|\varepsilon_{j}\right| \geq \frac{n-j}{\sqrt{n}} s_{n} \epsilon\right\}}\right] \\
& \leq C_{1} \frac{n}{\left[n-r_{n}\right]^{2}} \sum_{j=1}^{r_{n}} \mathbb{E}\left[\left(\varepsilon_{j}\right)^{2} \mathbb{I}_{\left.\left\{\left|\varepsilon_{j}\right| \geq \frac{n-r_{n}}{\sqrt{n}} \sqrt{C_{0}} \epsilon\right\}\right]}\right] \\
& =C_{1} \frac{n}{\left[n-r_{n}\right]^{2}} r_{n} \mathbb{E}\left[\left(\varepsilon_{1}\right)^{2} \mathbb{I}_{\left\{\left|\varepsilon_{1}\right| \geq \frac{n-r_{n}}{\sqrt{n}} \sqrt{C_{0}} \epsilon\right\}}\right]
\end{aligned}
$$

Temos que

$$
\begin{gathered}
C_{1} \frac{n}{\left[n-r_{n}\right]^{2}} r_{n}=\frac{n\lfloor n t\rfloor}{t \mathbb{E}\left[\left(\varepsilon_{1}\right)^{2}\right][n-\lfloor n t]]^{2}} \underset{n \rightarrow \infty}{\longrightarrow} \frac{1}{\mathbb{E}\left[\left(\varepsilon_{1}\right)^{2}\right](1-t)}, \\
\frac{n-r_{n}}{\sqrt{n}} \sqrt{C_{0}} \epsilon \underset{n \rightarrow \infty}{\longrightarrow}+\infty \Rightarrow \mathbb{I}_{\left\{\left|\varepsilon_{1}\right| \geq \frac{n-r_{n}}{\sqrt{n}} \sqrt{C_{0}} \epsilon\right\} \stackrel{q . c}{\rightarrow} 0} 0 .
\end{gathered}
$$

de (A.26), (A.27), (A.28) e o Teorema da convergência dominada, obtemos que

$$
\sum_{j=1}^{r_{n}} \frac{1}{s_{n}^{2}} \mathbb{E}\left[Y_{n k}^{2} \mathbb{I}_{\left\{\left|Y_{n k}\right| \geq s_{n} \epsilon\right\}}\right] \underset{n \rightarrow \infty}{\longrightarrow} 0 .
$$

da condição de Lindenberg, obtemos:

$$
\frac{\sum_{j=1}^{r_{n}} Y_{n j}}{s_{n}} \underset{n \rightarrow \infty}{\stackrel{\mathcal{D}}{\longrightarrow}} N(0,1)
$$

Como $s_{n}^{2}=\sum_{j=1}^{\lfloor n t\rfloor} \frac{n \mathbb{E}\left[\left(\varepsilon_{1}\right)^{2}\right]}{[n-j]^{2}}=2 \mathbb{E}\left[\varepsilon_{1}^{2}\right] \sum_{j=1}^{\lfloor n t\rfloor} \frac{1}{\left[1-\frac{j}{n}\right]^{2}} \frac{1}{n}$ portanto

$$
s_{n \rightarrow \infty}^{2} \underset{n \rightarrow}{\longrightarrow}\left[\mathbb{E}\left[\varepsilon_{1}^{2}\right] \int_{0}^{t} \frac{1}{[(1-x)]^{2}} d x=2 \mathbb{E}\left[\varepsilon_{1}^{2}\right] g(t)\right.
$$

Logo de (A.29), (A.30) e o Teorema de Slutski,

$$
\sum_{j=1}^{r_{n}} Y_{n j}=s_{n} \frac{\sum_{j=1}^{r_{n}} Y_{n j}}{s_{n}} \underset{n \rightarrow \infty}{\stackrel{\mathcal{D}}{\longrightarrow}} \sqrt{2 \mathbb{E}\left[\varepsilon_{1}^{2}\right]} N(0, g(t))
$$

Isto é,

$$
\tilde{X}_{t}^{n} \underset{n \rightarrow \infty}{\stackrel{\mathcal{D}}{\longrightarrow}} \sqrt{2 \mathbb{E}\left[\varepsilon_{1}^{2}\right]} N(0, g(t))
$$

como $f_{n}(\lfloor n t\rfloor) \underset{n \rightarrow \infty}{\longrightarrow}(1-t)=f(t)$, de (A.31), usando de novo o Teorema de Slutski, obtemos: 


$$
X_{t}^{n}=f_{n}(\lfloor n t\rfloor) \tilde{X}_{t}^{n} \underset{n \rightarrow \infty}{\stackrel{\mathcal{D}}{\longrightarrow}} c f(t) N(0, g(t))
$$

$\operatorname{com} c=\sqrt{2 \mathbb{E}\left[\varepsilon_{1}^{2}\right]}$.

Sejam $0<s<t \leq 1$ fixos, vejamos que

$$
\left(X_{s}^{n}, X_{t}^{n}\right) \underset{n \rightarrow \infty}{\stackrel{\mathcal{D}}{\longrightarrow}}(c f(s) N(0, g(s)), c f(t) N(0, g(t)))
$$

Consideremos o vetor aleatório

$$
\begin{aligned}
\left(X_{s}^{n}, X_{t}^{n}-X_{s}^{n}\right) & =\left(f_{n}(\lfloor n s\rfloor) \tilde{X}_{s}^{n}, f_{n}(\lfloor n t\rfloor) \tilde{X}_{t}^{n}-f_{n}(\lfloor n s\rfloor) \tilde{X}_{s}^{n}\right) \\
& =\left(f_{n}(\lfloor n s\rfloor) \tilde{X}_{s}^{n}, f_{n}(\lfloor n t\rfloor)\left(\tilde{X}_{t}^{n}-\tilde{X}_{s}^{n}\right)\right)+\left(0, \tilde{X}_{s}^{n}\left(f_{n}(\lfloor n t\rfloor)-f_{n}(\lfloor n s\rfloor)\right)\right) .
\end{aligned}
$$

Logo analogamente ao caso anterior e devido à independência das componentes do vetor, obtemos

$$
\begin{aligned}
\left(f_{n}(\lfloor n s\rfloor) \tilde{X}_{s}^{n}, f_{n}(\lfloor n t\rfloor)\left(\tilde{X}_{t}^{n}-\tilde{X}_{s}^{n}\right)\right) \underset{n \rightarrow \infty}{\stackrel{\mathcal{D}}{\longrightarrow}}\left(c f(s) N_{1}\left(0, g(s), c f(t) N_{2}\left(0, \int_{s}^{t} \frac{1}{(1-x)^{2}} d x\right)\right)\right. \\
=\left(c f(s) N_{1}(0, g(s)), c f(t) N_{2}(0, g(t)-g(s))\right)
\end{aligned}
$$

com $N_{1}, N_{2}$ independentes.

Também,

$$
\left(0, \tilde{X}_{s}^{n}\left(f_{n}(\lfloor n t\rfloor)-f_{n}(\lfloor n s\rfloor)\right)\right) \underset{n \rightarrow \infty}{\stackrel{\mathcal{D}}{\longrightarrow}}\left(0,(f(t)-f(s)) c N_{1}(0, g(s))\right) .
$$

Portanto, de (A.34),(A.35) temos que:

$$
\left(X_{s}^{n}, X_{t}^{n}-X_{s}^{n}\right) \underset{n \rightarrow \infty}{\stackrel{\mathcal{D}}{\rightarrow}}\left(c f(s) N_{1}(0, g(s)), c f(t) N_{2}(0, g(t)-g(s))\right)+\left(0, c[f(t)-f(s)] N_{1}(0, g(s))\right)
$$

Assim de (A.36), e a indepêndencia de $N_{1}, N_{2}$ obtemos 


$$
\begin{aligned}
\left(X_{s}^{n}, X_{t}^{n}\right) \quad & =\left(X_{s}^{n}, X_{t}^{n}-X_{s}^{n}\right)+\left(0, X_{s}^{n}\right) \\
\underset{n \rightarrow \infty}{\mathcal{D}} & \left(c f(s) N_{1}(0, g(s)), c f(t) N_{2}(0, g(t)-g(s))\right)+\left(0, c[f(t)-f(s)] N_{1}(0, g(s))\right) \\
& +\left(0, c f(s) N_{1}(0, g(s))\right) \\
= & \left(c f(s) N_{1}(0, g(s)), c f(t) N_{2}(0, g(t)-g(s))\right)+\left(0, c f(t) N_{1}(0, g(s))\right) \\
= & \left(c f(s) N_{1}(0, g(s)), c f(t) N_{3}(0, g(t))\right)
\end{aligned}
$$

Analogamente se mostra que dados $m \in \mathbb{N}$ e $0 \leq t_{1} \leq \ldots \leq t_{m} \leq 1$ fixos, temos que

$$
\left(X_{t_{1}}^{n}, \ldots, X_{t_{m}}^{n}\right) \underset{n \rightarrow \infty}{\stackrel{\mathcal{D}}{\longrightarrow}}\left(c f\left(t_{1}\right) N_{1}\left(0, g\left(t_{1}\right)\right), \ldots, c f\left(t_{m}\right) N_{m}\left(0, g\left(t_{m}\right)\right)\right)
$$

de (A.38) segui o resultado.

Lema A.0.3 Sejam $X^{n}$ como em (A.11), $X$ como em (A.10) com $c=\sqrt{2 \mathbb{E}\left[\varepsilon_{1}^{2}\right]}$, então $X^{n} \underset{n \rightarrow \infty}{\stackrel{\mathcal{D}}{\rightarrow}} X$ em $D_{[0,1-\alpha]}$.

Prova: Segue do Teoremas A.0.1 e os Lemas A.0.1 e A.0.2.

Teorema A.0.2 Seja $X^{n}$ o processo em (A.11), X o processo em (A.10) com $c=\sqrt{2 \mathbb{E}\left[\varepsilon_{1}^{2}\right]}$. Considere o processo em $C_{[0,1-\alpha]}$ definido por

$$
Z_{t}^{n}=Y_{n}(\lfloor n t\rfloor)+(n t-\lfloor n t\rfloor)\left(Y_{n}(\lfloor n t\rfloor+1)-Y_{n}(\lfloor n t\rfloor)\right), \quad t \in[0,1-\alpha]
$$

Então

$$
Z_{n} \underset{n \rightarrow \infty}{\stackrel{\mathcal{D}}{\longrightarrow}} X \quad \text { em } C_{[0,1-\alpha]}
$$

Prova: Segue da Proposição A.0.1 e o Teorema anterior. 


\section{Apêndice B}

\section{Alguns resultados}

Seja $\left(Q_{k}\right)_{k=0}^{k_{n}}$ processos de Poisson independentes. Suponha que $Q_{k}$ tem taxa $\frac{n-k}{n} \operatorname{com} Q_{k}=\sqrt{n} R_{k}$. Seja $r>0$ fixo, consideremos o conjunto $\mathbb{Z}_{r}:=\{r k: k \in \mathbb{Z}\}$. Para $k=0,1,2, \ldots, k_{n}$, definimos $E_{k}=E_{k}(r)=\mathbb{Z}_{r} \times\left\{l_{k}\right\}, l_{k}=\frac{n k}{n-k}$ e $E=\cup_{k=0}^{k_{n}} E_{k}$. Dizemos que um ponto $z_{k}=\left(r j, l_{k}\right) \in E_{k}$, para algum $j \in \mathbb{Z}$ esta aberto se $Q_{k} \cap[r j, r(j+1)) \neq \emptyset$ e esta fechado caso contrario. Portanto, se $p_{k}^{r}$ é a probabilidade de $z_{k}$ estar aberto temos que:

$$
p_{k}^{r}=\mathbb{P}\left[Q_{k} \cap[r j, r(j+1)) \neq \emptyset\right]=1-\mathbb{P}\left[Q_{k} \cap[r j, r(j+1))=\emptyset\right]=1-e^{-\frac{n-k}{n} r} .
$$

Seja $\Omega^{n, r}=(\omega(z), z \in E)$ uma familia de variáveis aleatórias Bernoullis independentes tais que no nível $l_{k}$, tem parâmetro $p_{k}^{r}$, para $k=0,1,2, \ldots, k_{n}$. Consideremos uma segunda familia, $\Gamma=$ $\left(v\left(z_{k}\right), z_{k} \in E_{k}\right)_{0 \leq k \leq k_{n}-1}$ de variáveis aleatórias independentes Bernoulli's de parâmetro 1/2.

Para $z_{k}=\left(a_{k}, l_{k}\right) \in E_{k},\left(k<k_{n}\right)$ seja $h_{k}\left(z_{k}\right)=\arg \min _{z^{\prime} \in E_{k+1}}\left\|z-z^{\prime}\right\|$ e $w\left(h_{k}(z)\right)=1$. Se existem dois vértices mais próximos a $z_{k}$ no nível seguinte, então a ligação será a seguinte, se $v\left(z_{k}\right)=1$ fazemos $h_{k}\left(z_{k}\right)$ igual ao ponto da direita e se $v\left(z_{k}\right)=0$ fazemos $h_{k}\left(z_{k}\right)$ igual ao ponto da esquerda.

Seja $h_{k}^{0}\left(z_{k}\right)=z_{k}$ e indutivamente, para $k \leq j \leq k_{n}-k$ seja

$$
h_{k}^{j}\left(z_{k}\right)=\arg \min _{z^{\prime} \in E_{k+j}}\left\|h_{k}^{(j-1)}\left(z_{k}\right)-z^{\prime}\right\| \wedge w\left(h_{k}^{j}\left(z_{k}\right)\right)=1 .
$$

Considere $\mathcal{G}_{r}=(V, \mathcal{E})$ o grafo orientado com vértices $V=E$ e elos $\mathcal{E}=\left\{\left(u, h_{k}^{1}(u)\right): u \in E_{k}\right\}_{k=0}^{k_{n}}$.

Dado $a \in \mathbb{R}$, sejam $m_{r}(a)=r\left\lfloor\frac{a}{r}\right\rfloor$ e $\tilde{X}_{a}^{r}$ o caminho de $\mathcal{G}_{r}$ começando do ponto $\left(m_{r}(a), 0\right)$.

Dado $a \in\left[-n^{\delta / 2+1 / 2}, n^{\delta / 2+1 / 2}\right], K_{a}^{n}:=\left\{\left(\sqrt{n} x_{1}, n x_{2}\right):\left(x_{1}, x_{2}\right) \in \hat{Y}_{(a / \sqrt{n}, 0)}^{n, T}\right\}$. Escrevemos $\hat{Y}_{(a / \sqrt{n}, 0)}^{n, T}$ como um abuso de notação, pois quase certamente o ponto $(a / \sqrt{n}, 0) \notin R_{0}$. 
Observe agora que os incrementos de $K_{a}^{n}$ são devidos aos processos de Poisson $Q_{k}=\sqrt{n} R_{k}$ com $\operatorname{taxas} \frac{n-k}{n}, k=0,1,2, \ldots, k_{n}$.

Seja $t \in(0,1 / \alpha-1)$ fixo. Seja $\tilde{\Pi}_{a}^{r}$ o espaço de estados de $\tilde{X}_{a}^{r}$. Considere a seguinte ordem parcial $\prec$ sobre $\tilde{\Pi}_{a}^{r}$. Dados $\pi_{1}^{r}, \pi_{2}^{r} \in \tilde{\Pi}_{a}^{r}$, dizemos que

$$
\pi_{1}^{r} \prec \pi_{2}^{r} \Leftrightarrow \pi_{1}^{r}\left(l_{k}\right)-\pi_{1}^{r}\left(l_{s}\right) \leq \pi_{2}^{r}\left(l_{k}\right)-\pi_{2}^{r}\left(l_{s}\right),
$$

para todo $\lfloor n t\rfloor \geq k \geq s \geq 0$.

Proposição B.0.2 Sejam $\pi_{1}^{r}, \pi_{2}^{r} \in \tilde{\Pi}_{a}^{r}$ tais que $\pi_{1}^{r} \prec \pi_{2}^{r}$. Então, para $x<a<y$

$$
\mathbb{P}\left[\tilde{X}_{x}^{r}\left(l_{\lfloor n t\rfloor}\right)<\tilde{X}_{a}^{r}\left(l_{\lfloor n t\rfloor}\right) \mid \tilde{X}_{a}^{r}=\pi_{1}^{r}\right] \leq \mathbb{P}\left[\tilde{X}_{x}^{r}\left(l_{\lfloor n t\rfloor}\right)<\tilde{X}_{a}^{r}\left(l_{\lfloor n t\rfloor}\right) \mid \tilde{X}_{a}^{r}=\pi_{2}^{r}\right]
$$

$e$

$$
\mathbb{P}\left[\tilde{X}_{y}^{r}\left(l_{\lfloor n t\rfloor}\right)>\tilde{X}_{a}^{r}\left(l_{\lfloor n t\rfloor}\right) \mid \tilde{X}_{a}^{r}=\pi_{1}^{r}\right] \geq \mathbb{P}\left[\tilde{X}_{y}^{r}\left(l_{\lfloor n t\rfloor}\right)>\tilde{X}_{a}^{r}\left(l_{\lfloor n t\rfloor}\right) \mid \tilde{X}_{a}^{r}=\pi_{2}^{r}\right]
$$

Prova: Se $m_{r}(x)=m_{r}(a)$ então $\tilde{X}_{x}^{r}=\tilde{X}_{a}^{r}$ e portanto para toda realização $\pi^{r}$ de $\tilde{\Pi}_{a}^{r}$ segui que

$$
\mathbb{P}\left[\tilde{X}_{x}^{r}\left(l_{\lfloor n t\rfloor}\right)<\tilde{X}_{a}^{r}\left(l_{\lfloor n t\rfloor}\right) \mid \tilde{X}_{a}^{r}=\pi^{r}\right]=0
$$

o qual mostra (B.4). Se $m_{r}(x)<m_{r}(a)$ então veja [2] pagina 8. Analogamente se prova (B.5).

Observação B.0.2 A prova em [2] apresenta um único argumento diferente, isto é, para fazer os acoplamentos e os argumentos de dominação estocastica em nosso caso nos movimentamos $r$ unidades a direita ou $r$ unidades a esquerda enquanto que em [2] se movimentam 1 unidade a direita ou 1 unidade a esquerda.

Lema B.0.4 Seja $a \in\left[-n^{\delta / 2+1 / 2}, n^{\delta / 2+1 / 2}\right]$ fixo. Então

$$
\tilde{X}_{a}^{r}\left(l_{k}\right) \underset{r \rightarrow 0_{+}}{\stackrel{q . c}{\rightarrow}} K_{a}^{n}\left(l_{k}\right)
$$

$\operatorname{para} k=0,1, \ldots, k_{n}$.

Prova: Como $m_{r}(a)=r\left\lfloor\frac{a}{r}\right\rfloor$ então $m_{r}(a)=r\left\lfloor\frac{a}{r}\right\rfloor \underset{r \rightarrow 0_{+}}{\longrightarrow}$ a, assim $\tilde{X}_{a}^{r}(0) \underset{r \rightarrow 0_{+}}{\stackrel{q \cdot c}{\longrightarrow}} K_{a}^{n}(0)$.

Definimos, $M_{k}=\left|K_{h}^{n}\left(l_{k}\right)-\tilde{X}_{h}^{r}\left(l_{k}\right)\right|$ para $0 \leq k \leq k_{n}$ e seja $B_{k}$ o evento $M_{k}>r$ mais $M_{j} \leq r$ para $2 \leq j<k$. Então, 


$$
\mathbb{P}\left[\max _{1 \leq k \leq k_{n}} M_{k}>r\right]=\mathbb{P}\left[M_{1}>r\right]+\sum_{k=2}^{k_{n}} \mathbb{P}\left[B_{k}\right]
$$

Seja $q_{r, k}$ a probabilidade de ter dois pontos diferentes em $u, v \in \mathbb{Z}_{r} \times\left\{l_{k}\right\}$, equidistantes de $\left(0, l_{k}\right)$, com $u=\left(u_{1}, l_{k}\right), v=\left(v_{1}, l_{k}\right)$ e $u_{1}<v_{1}$ tais que $\omega(u)=\omega(v)=1$ e $\omega(z)=0$ para todo $z=\left(z_{1}, l_{k}\right) \in$ $\mathbb{Z}_{r} \times\left\{l_{k}\right\}$ com $u_{1}<z_{1}<v_{1}$. Pela invariância por translação temos que

$$
\mathbb{P}\left[M_{1}>r\right] \leq q_{r, 1} \quad \text { e } \quad \mathbb{P}\left[B_{k}\right] \leq q_{k, r}
$$

segue de (B.7) e (B.8) que

$$
\mathbb{P}\left[\max _{1 \leq k \leq k_{n}} M_{k}>r\right] \leq \sum_{k=1}^{k_{n}} q_{k, r} .
$$

Agora, $q_{k, r}$ converge quando $r \rightarrow 0_{+} \grave{a}$ probabilidade de ter dois pontos consecutivos do processo de Poisson $Q_{k}$ equidistantes à origem, portanto $q_{k, r} \underset{r \rightarrow 0_{+}}{\longrightarrow} 0$. Logo,

$$
\mathbb{P}\left[\max _{1 \leq k \leq k_{n}} M_{k}>r\right] \underset{r \rightarrow 0_{+}}{\longrightarrow} 0
$$

ou equivalentemente

$$
\tilde{X}_{a}^{r}\left(l_{k}\right) \underset{r \rightarrow 0_{+}}{\stackrel{q . c}{\longrightarrow}} K_{a}^{r}\left(l_{k}\right), \quad k=0,1, \ldots, k_{n}
$$

Teorema B.0.3 Sejam U, V variáveis aleatórias que assumem seus valores em $\left(S_{1}, \mathfrak{S}_{1}\right)$ e $\left(S_{2}, \mathfrak{S}_{2}\right)$ respectivamente. Seja $\varphi$ uma função mensurável sobre $\left(S_{1} \times S_{2}, \mathfrak{S}_{1} \otimes \mathfrak{S}_{2}\right)$ que assume valores reais. Se $U$ é $\mathfrak{G}$ mensurável, $\sigma(V)$ e $\mathfrak{G}$ são independentes, e $\mathbb{E}[\varphi(U, V)]<\infty$, então temos que:

$$
\mathbb{E}[\varphi(U, V) \mid \mathfrak{G}]=h(U) \quad \text { onde } \quad h(u):=\mathbb{E}[\varphi(u, V)]
$$

Prova: $\operatorname{Ver}[7]$ pag. 27

Proposição B.0.3 Seja $W(t)$ um movimento Browniano começando em zero. Se $\tau=\inf \{t \geq 0$ : $W(t)>0\}$, então $\mathbb{P}[\tau=0]=1$.

Prova: Ver [6] pág 384. 
Observação B.0.3 A Proposição B.0.3 implica que o movimento Browniano começando desde a origem atinge $(0, \infty)$ imediatamente e analogamente atinge $(-\infty, 0)$ imediatamente. 


\section{Referências Bibliográficas}

[1] Fontes, Isopi, Newman and Ravishankar, The Brownian web: characterization and convergence, Ann. Probab 32 (2004), 2857-2883. 2, 8

[2] Coletti, Fontes and Dias, Scaling limits for a drainage network model, J. Appl. Probab 46 no 4 (2009), 1184-1197. 38, 45, 46, 51, 68

[3] Ferrari, Fontes and Wu, Two-dimensional Poisson trees converge to the Brownian web, Ann. Inst. H. Poincar Probab. Statist, 41 (2005), 851-858.

[4] P. Billingsley, Convergence of Probability Measures, Wiley Series In Probability And Statistics, 1999. 58

[5] Stewart N. Ethier and Thomas G. Kurtz, Markov Processes: Characterization and convergence, Wiley Series In Probability And Statistics, 2005. 58

[6] R. Durret, Probability: Theory and examples, Duxbury Press. Second Edition, 1996. 40, 43, 69

[7] Rabi Bhattacharya and Edward C. Waymire, A Basic Course In Probability Theory, Springer, 2007. 69

[8] Baccelli and Bordenave, The radial spanning tree of a Poisson point process, Ann. Probab 17 (2007), 305-359. 1 